\title{
Vanishing viscosity limit of the three-dimensional barotropic compressible Navier-Stokes equations with degenerate viscosities and far-field vacuum
}

\author{
Geng Chen, Gui-Qiang G. Chen, and Shengguo Zhu
}

\begin{abstract}
We are concerned with the inviscid limit of the Navier-Stokes equations to the Euler equations for barotropic compressible fluids in $\mathbb{R}^{3}$. When the viscosity coefficients obey a lower power law of the density (i.e., $\rho^{\delta}$ with $0<\delta<1$ ), we identify a quasi-symmetric hyperbolic-singular elliptic coupled structure of the Navier-Stokes equations to control the behavior of the velocity of the fluids near a vacuum. Then this structure is employed to prove that there exists a unique regular solution to the corresponding Cauchy problem with arbitrarily large initial data and far-field vacuum, whose life span is uniformly positive in the vanishing viscosity limit. Some uniform estimates on both the local sound speed and the velocity in $H^{3}\left(\mathbb{R}^{3}\right)$ with respect to the viscosity coefficients are also obtained, which lead to the strong convergence of the regular solutions of the Navier-Stokes equations with finite mass and energy to the corresponding regular solutions of the Euler equations in $L^{\infty}\left([0, T] ; H_{\mathrm{loc}}^{s}\left(\mathbb{R}^{3}\right)\right)$ for any $s \in[2,3)$. As a consequence, we show that, for both viscous and inviscid flows, it is impossible that the $L^{\infty}$ norm of any global regular solution with vacuum decays to zero asymptotically, as $t$ tends to infinity. Our framework developed here is applicable to the same problem for other physical dimensions via some minor modifications.
\end{abstract}

\section{Contents}

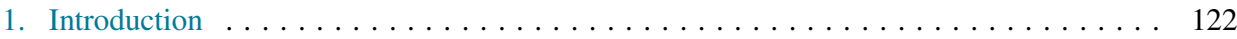

2. Main theorems . . . . . . . . . . . . . . . . . . . . . . . . . . . . . . 129

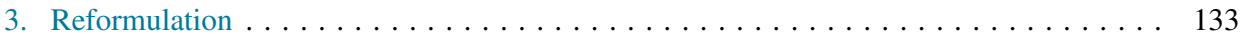

4. Uniform energy estimates for the reformulated problem . . . . . . . . . . . . . . 136

5. Proof of Theorem $2.1 \ldots \ldots \ldots \ldots \ldots \ldots \ldots \ldots \ldots$

6. Vanishing viscosity limit as $\varepsilon \rightarrow 0 \ldots \ldots \ldots \ldots \ldots \ldots \ldots$

7. Nonexistence of global solutions with $L^{\infty}$ decay $\ldots \ldots \ldots \ldots \ldots \ldots$

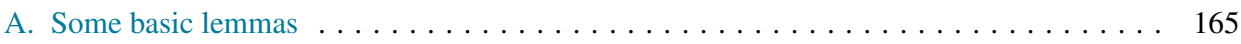

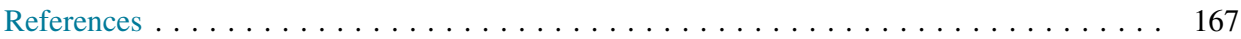

2020 Mathematics Subject Classification. 35Q30, 35B40, 35A09, 35D35, 35D40.

Keywords. Compressible Navier-Stokes equations, vanishing viscosity limit, three dimensions, far-field vacuum, degenerate viscosity, regular solutions, degenerate parabolicity. 


\section{Introduction}

The time evolution of the density $\rho^{\varepsilon} \geq 0$ and velocity $u^{\varepsilon}=\left(\left(u^{\varepsilon}\right)^{(1)},\left(u^{\varepsilon}\right)^{(2)},\left(u^{\varepsilon}\right)^{(3)}\right)^{\top}$ $\in \mathbb{R}^{3}$ of a general viscous barotropic compressible fluid in $\mathbb{R}^{3}$ is governed by the following compressible Navier-Stokes equations (CNS):

$$
\left\{\begin{array}{l}
\rho_{t}^{\varepsilon}+\operatorname{div}\left(\rho^{\varepsilon} u^{\varepsilon}\right)=0, \\
\left(\rho^{\varepsilon} u^{\varepsilon}\right)_{t}+\operatorname{div}\left(\rho^{\varepsilon} u^{\varepsilon} \otimes u^{\varepsilon}\right)+\nabla p\left(\rho^{\varepsilon}\right)=\varepsilon \operatorname{div} \mathbb{T}\left(\rho^{\varepsilon}, \nabla u^{\varepsilon}\right),
\end{array}\right.
$$

where $\varepsilon>0$ is a constant measuring the strength of the viscosity, which is assumed to be $\varepsilon \in(0,1]$ without loss of generality, and $x=\left(x_{1}, x_{2}, x_{3}\right) \in \mathbb{R}^{3}$ and $t \geq 0$ are the space and time variables, respectively. For polytropic gases, the constitutive relation is given by

$$
p(\rho)=A \rho^{\gamma} \quad \text { with } A>0 \text { and } \gamma>1,
$$

where $A$ is an entropy constant and $\gamma$ is the adiabatic exponent. The viscous stress tensor $\mathbb{T}(\rho, \nabla u)$ has the form

$$
\mathbb{T}(\rho, \nabla u)=\mu(\rho)\left(\nabla u+(\nabla u)^{\top}\right)+\lambda(\rho) \operatorname{div} u \mathbb{I}_{3},
$$

where $\mathbb{I}_{3}$ is the $3 \times 3$ identity matrix,

$$
\mu(\rho)=\alpha \rho^{\delta}, \quad \lambda(\rho)=\beta \rho^{\delta}
$$

for some constant $\delta>0, \mu(\rho)$ is the shear viscosity coefficient, $\lambda(\rho)+\frac{2}{3} \mu(\rho)$ is the bulk viscosity coefficient, and $\alpha$ and $\beta$ are both constants satisfying

$$
\alpha>0, \quad \alpha+\beta \geq 0 .
$$

Formally, when $\varepsilon=0$, the Navier-Stokes equations (1.1) reduce to the compressible Euler equations for inviscid flow:

$$
\left\{\begin{array}{l}
\rho_{t}+\operatorname{div}(\rho u)=0, \\
(\rho u)_{t}+\operatorname{div}(\rho u \otimes u)+\nabla p(\rho)=0,
\end{array}\right.
$$

where $\rho$ and $u$ are the mass density and velocity of the inviscid fluid.

In this paper, we focus on the lower power-law case $\delta \in(0,1)$ to analyze the asymptotic behavior of the smooth solution $\left(\rho^{\varepsilon}, u^{\varepsilon}\right)$ with finite mass and energy as $\varepsilon \rightarrow 0$ for the Cauchy problem (1.1)-(1.5) with the following initial data and far-field vacuum:

$$
\begin{array}{ll}
\left.\left(\rho^{\varepsilon}, u^{\varepsilon}\right)\right|_{t=0}=\left(\rho_{0}^{\varepsilon}, u_{0}^{\varepsilon}\right)(x) & \text { with } \rho_{0}^{\varepsilon}(x)>0 \text { for } x \in \mathbb{R}^{3}, \\
\left(\rho_{0}^{\varepsilon}, u_{0}^{\varepsilon}\right)(x) \rightarrow(0,0) & \text { as }|x| \rightarrow \infty .
\end{array}
$$

Our results show that the inviscid flow (1.6) can be regarded as the viscous flow (1.1) with vanishing viscosity for the regular solutions with far-field vacuum in the sense of Definition 2.1. The far-field behavior

$$
\left(\rho^{\varepsilon}, u^{\varepsilon}\right)(t, x) \rightarrow(0,0) \quad \text { as }|x| \rightarrow \infty \text { for } t \geq 0
$$


is natural when some physical requirements are imposed, for example, when the total mass is finite in $\mathbb{R}^{3}$.

In the theory of gas dynamics, system (1.1) may be derived from the Boltzmann equation through the Chapman-Enskog expansion; see Chapman-Cowling ([8]) and Li-Qin ([34]). For some physical situations, the viscosity coefficients $\mu$ and $\lambda$ and the heat conductivity coefficient $\kappa$ are functions of the absolute temperature $\theta$ (cf. [8]) such as

$$
\mu(\theta)=a_{1} \theta^{\frac{1}{2}} F(\theta), \quad \lambda(\theta)=a_{2} \theta^{\frac{1}{2}} F(\theta), \quad \kappa(\theta)=a_{3} \theta^{\frac{1}{2}} F(\theta)
$$

for some constants $a_{i}, i=1,2,3$. In fact, for the cut-off inverse power force model, if the intermolecular potential varies as $r^{-a}$, then

$$
F(\theta)=\theta^{b} \quad \text { with } b=\frac{2}{a} \in[0, \infty),
$$

where $r$ is the intermolecular distance. In particular, $a=1$ and $b=2$ for ionized gas, $a=4$ and $b=\frac{1}{2}$ for Maxwellian molecules, and $a=\infty$ and $b=0$ for rigid elastic spherical molecules; see [8, $\S 10]$. As a typical example where $F$ is not a power function of $\theta$, Sutherland's model is well known:

$$
F(\theta)=\frac{\theta}{\theta+s_{0}},
$$

where $s_{0}>0$ is the Sutherland constant. According to Liu-Xin-Yang ([40]), for barotropic and polytropic fluids, such a dependence is inherited through the laws of Boyle and GayLussac:

$$
p=R \rho \theta=A \rho^{\gamma} \quad \text { for constant } R>0 ;
$$

that is, $\theta=A R^{-1} \rho^{\gamma-1}$ so that the viscosity coefficients are functions of the density. For most physical processes, $\gamma$ is in the interval $(1,3)$, which implies that $\delta \in(0,1)$ for rigid elastic spherical molecules. In this sense, the lower power-law case is the most physically relevant for degenerate viscous flow. In this paper we focus on this case. In fact, similar models with density-dependent viscosity coefficients arise from various physical situations, such as the Korteweg system, the shallow water equations, the lake equations, and quantum Navier-Stokes systems (cf. [2-7, 28, 30]).

Another motivation of our study is that the mathematical structure of system (1.1) is an excellent prototype of nonlinear degenerate systems of partial differential equations, since the equations on the time evolution of the fluid velocity become very singular near the far-field due to the decay of the density at a certain rate, which will be further discussed later.

\subsection{Well-posedness for compressible flow with vacuum}

We first recall some related frameworks on the well-posedness for strong solutions with vacuum of the Cauchy problem of the hydrodynamics equations mentioned above. For the inviscid flow, in Makino-Ukai-Kawashima ([43]), the local sound speed $c$ was first introduced to rewrite the system in a symmetric hyperbolic form, and the local existence of the 
unique regular solution of the compressible full Euler equations with vacuum was established; see also Makino-Perthame ([42]) for the Euler-Poisson equations. For isentropic flow in $\mathbb{R}^{3}$, the sound speed $c$ is defined by

$$
c:=\sqrt{p^{\prime}(\rho)}=\sqrt{A \gamma} \rho^{\frac{\gamma-1}{2}} .
$$

Then the result in [43] can be read as follows:

Theorem 1.1 ([43]). Let $\gamma>1$. For the Cauchy problem (1.6) with

$$
\left.(\rho, u)\right|_{t=0}=\left(\rho_{0}, u_{0}\right)(x) \text { for } x \in \mathbb{R}^{3},
$$

if the initial data $\left(\rho_{0}, u_{0}\right)$ satisfy

$$
\rho_{0}(x) \geq 0, \quad\left(c_{0}, u_{0}\right) \in H^{3}\left(\mathbb{R}^{3}\right),
$$

then there exist $T_{0}>0$ and a unique regular solution $(\rho, u)$ of the Cauchy problem (1.6) with (1.13) satisfying

$$
(c, u) \in C\left(\left[0, T_{0}\right] ; H^{3}\left(\mathbb{R}^{3}\right)\right), \quad\left(c_{t}, u_{t}\right) \in C\left(\left[0, T_{0}\right] ; H^{2}\left(\mathbb{R}^{3}\right)\right),
$$

where the regular solution $(\rho, u)$ is defined in the following sense:

(i) $(\rho, u)$ satisfies (1.6) with (1.13) in the sense of distributions;

(ii) $\rho \geq 0$ and $(c, u) \in C^{1}\left(\left[0, T_{0}\right] \times \mathbb{R}^{3}\right)$;

(iii) $u_{t}+u \cdot \nabla u=0$ when $\rho(t, x)=0$.

The corresponding global well-posedness of smooth solutions with small density but possibly large velocity in some homogeneous Sobolev spaces was proved in GrassinSerre ([21,49]). Chen-Chen-Zhu ([9]) pinpointed the necessary and sufficient condition for the formation of singularities of $C^{1}$ solutions with large initial data allowing a far-field vacuum for one-dimensional space: there exists a compression in the initial data.

For compressible viscous flow away from a vacuum, the local existence and uniqueness of classical solutions are known in Nash ([46]) and Serrin ([50]). However, if the initial density does not have a strictly positive lower bound, the arguments used in $[46,50]$ cannot apply to system (1.1), owing to the degeneracy caused by the vacuum or the decay of the density in the far-field in the momentum equations $(1.1)_{2}$ such as

$$
\underbrace{\rho^{\varepsilon}\left(u_{t}^{\varepsilon}+u^{\varepsilon} \cdot \nabla u^{\varepsilon}\right)}_{\text {degeneracy of time evolution }}+\nabla p\left(\rho^{\varepsilon}\right)=\varepsilon \underbrace{\operatorname{div}\left(2 \mu\left(\rho^{\varepsilon}\right) D\left(u^{\varepsilon}\right)+\lambda\left(\rho^{\varepsilon}\right) \operatorname{div} u^{\varepsilon} \mathbb{I}_{3}\right)}_{\text {degeneracy of viscosities }},
$$

where $D(u)=\frac{1}{2}\left(\nabla u+(\nabla u)^{\top}\right)$. For constant viscous flow (i.e., $\delta=0$ in (1.4)), in order to establish the local well-posedness of strong solutions with vacuum in $\mathbb{R}^{3}$, a remedy was suggested by Cho-Choe-Kim ([13]) for dealing with the degeneracy of time evolution, where they imposed initially a compatibility condition:

$$
\nabla p\left(\rho_{0}^{\varepsilon}\right)-\operatorname{div} \mathbb{T}_{0}=\sqrt{\rho_{0}^{\varepsilon}} g \quad \text { for some } g \in L^{2}\left(\mathbb{R}^{3}\right) .
$$


Later, based on the uniform estimate of the upper bound of the density, Huang-Li-Xin ([26]) extended this solution to be a global one with small energy for barotropic flow in $\mathbb{R}^{3}$.

For degenerate viscous flow (i.e., $\delta>0$ in (1.4)), the strong degeneracy of the momentum equations in (1.1) near the vacuum causes new difficulties for the mathematical analysis of this system. Based on the B-D entropy introduced by Bresch-Desjardins $([2,3])$, some significant results on weak solutions of the isentropic CNS or related models whose viscosity coefficients satisfy the B-D relation have been obtained; see $[4,5,33$, $45,53]$. However, owing to the degenerate mathematical structure and lack of smooth effects on the solutions when a vacuum appears, many fundamental questions remain open, including the uniform estimate of the life span of the corresponding strong solutions with respect to $\varepsilon$, identification of the classes of initial data that either cause the finite time blow-up or provide the global existence of strong solutions, and the well-posedness of solutions with vacuum for system (1.1) without the B-D relation for the viscosity coefficients.

In fact, in $[13,26]$, the uniform ellipticity of the Lamé operator $L$ defined by

$$
L u:=-\alpha \Delta u-(\alpha+\beta) \nabla \operatorname{div} u
$$

plays an essential role in improving the regularity of $u^{\varepsilon}$, which can be shown as

$$
\left\|\nabla^{k+2} u^{\varepsilon}\right\|_{L^{2}\left(\mathbb{R}^{3}\right)} \leq C \varepsilon^{-1}\left\|\nabla^{k}\left(\rho^{\varepsilon} u_{t}^{\varepsilon}+\rho^{\varepsilon} u^{\varepsilon} \cdot \nabla u^{\varepsilon}+\nabla p\left(\rho^{\varepsilon}\right)\right)\right\|_{L^{2}\left(\mathbb{R}^{3}\right)}
$$

for $k=0,1,2$, and some constant $C>0$ independent of $\varepsilon$. However, when $\delta>0$ in (1.4), the viscosity coefficients approach zero continuously near the vacuum. This degeneracy makes it difficult to adapt the elliptic approach (1.16) to the present case. Moreover, we need to pay additional attention to deal with the strong nonlinearity of the variable coefficients of the viscous term due to $\delta>0$, which is another crucial issue owing to the appearance of a vacuum or the density decay in the far-field.

Recently, when $0<\delta<1$, under the initial compatibility conditions

$$
\nabla u_{0}^{\varepsilon}=\left(\rho_{0}^{\varepsilon}\right)^{\frac{1-\delta}{2}} g_{1}, \quad L u_{0}^{\varepsilon}=\left(\rho_{0}^{\varepsilon}\right)^{1-\delta} g_{2}, \quad \nabla\left(\left(\rho_{0}^{\varepsilon}\right)^{\delta-1} L u_{0}^{\varepsilon}\right)=\left(\rho_{0}^{\varepsilon}\right)^{\frac{1-\delta}{2}} g_{3}
$$

for some $\left(g_{1}, g_{2}, g_{3}\right) \in L^{2}\left(\mathbb{R}^{3}\right)$, the existence of the unique local classical solution with far-field vacuum to (1.1) in $\mathbb{R}^{3}$ was established in Xin-Zhu ([55]) by introducing an elaborate singular elliptic approach on the two operators $L u$ and $L\left(\rho^{\delta-1} u\right)$. For the case $\delta=1$, in Li-Pan-Zhu ([36]), it was first observed that the degeneracies of the time evolution and viscosities can be transferred to the possible singularity of the term $\nabla \log \rho \cdot \nabla u$. Then, by establishing some uniform estimates of $\nabla \log \rho^{\varepsilon}$ in $L^{6}\left(\mathbb{R}^{3}\right)$ and $\nabla^{2} \log \rho^{\varepsilon}$ in $H^{1}\left(\mathbb{R}^{3}\right)$ with respect to the lower bound of the initial density, the existence of the unique local classical solution with far-field vacuum of system (1.1) in $\mathbb{R}^{2}$ was obtained, which also applies to the two-dimensional shallow water equations. Later, by introducing a hyperbolic approach and making full use of weak smooth effect on the 
solutions of system (1.1) to establish some weighted estimates on the highest-order term $\nabla^{4} u^{\varepsilon}$, the existence of three-dimensional local classical solutions was obtained in [37] when $1<\delta \leq \min \left\{3, \frac{\gamma+1}{2}\right\}$, and the corresponding global well-posedness in some homogeneous Sobolev spaces was established by Xin-Zhu ([54]) under some initial smallness assumptions. See also [14,23,32,35,38,56-59] for other related results.

\subsection{Vanishing viscosity limit}

Based on the well-posedness theory mentioned above, a natural follow-up question is to understand the relation between the regular solutions of inviscid flow in [43] and those of viscous flow for $\delta \geq 0$ in $[13,36,37,54,55]$ with vanishing physical viscosities, especially for the hard sphere model when $0<\delta<1$.

There is a considerable literature on the uniform bounds and the vanishing viscosity limit in the whole space. The idea of regarding inviscid flow as viscous flow with vanishing physical viscosity dates back to Hugoniot ([27]), Rankine ([47]), Rayleigh ([48]), and Stokes ([52]); see Dafermos ([15]). However, it was only in 1951 that Gilbarg ([20]) gave the first rigorous convergence analysis of vanishing physical viscosity limit from the Navier-Stokes equations (1.1) to the isentropic Euler equations (1.6), and established the mathematical existence and vanishing viscous limit of the Navier-Stokes shock layers. The framework on the convergence analysis of piecewise smooth solutions was established by Gùes-Métivier-Williams-Zumbrun ([22]), Hoff-Liu ([24]), and the references cited therein. Klainerman-Majda ([29]) established the convergence of smooth solutions of the Navier-Stokes equations to solutions of the Euler equations in $H^{s}\left(\mathbb{R}^{d}\right)$ for $s>\frac{d}{2}+1$. In 2009, combining the uniform energy estimates with compactness compensated arguments, Chen-Perepelitsa ([10]) provided the first rigorous proof of the vanishing physical viscosity limit of solutions of the one-dimensional Navier-Stokes equations to a finite-energy entropy solution of the isentropic Euler equations with relative finite-energy initial data. Some further results can be found in Bianchini-Bressan ([1]) for strictly hyperbolic systems with artificial viscosity, Chen-Perepelitsa ([11]) and Chen-Schrecker ([12]) for the spherically symmetric case with artificial viscosity, as well as Huang-Pan-WangWang-Zhai ([25]) and Germain-LeFloch ([19]).

However, even in the one-dimensional case, owing to the complex mathematical structure of the hydrodynamics equations near a vacuum, the existence results for strong solutions for the viscous and inviscid cases are often established in totally different frameworks, such as $[13,36,37,55]$ for viscous flow and $[9,43]$ for inviscid flow. In fact, the arguments used in $[13,26,36,37,55]$ essentially rely on the uniform ellipticity of the Lamé operator or some related elliptic operators, and both the desired a priori estimates of the solutions and the life spans $T^{\varepsilon}$ depend strictly on the real physical viscosities. For example,

- $\quad$ when $\delta=0$ in $[13,26], T^{\varepsilon}=O(\varepsilon)$ and (1.16); 
- when $0<\delta<1$ in [55], $T^{\varepsilon}=O(\varepsilon)$ and

$$
\begin{aligned}
& \left\|\nabla^{k+2}\left(\left(\rho^{\varepsilon}\right)^{\delta-1} u^{\varepsilon}\right)\right\|_{L^{2}\left(\mathbb{R}^{3}\right)} \\
& \leq C \varepsilon^{-1}\left\|\nabla^{k}\left(u_{t}^{\varepsilon}+u^{\varepsilon} \cdot \nabla u^{\varepsilon}+A \gamma\left(\rho^{\varepsilon}\right)^{\gamma-2} \nabla \rho^{\varepsilon}\right)\right\|_{L^{2}\left(\mathbb{R}^{3}\right)} \\
& \quad+C\left(\left\|\nabla^{k}\left(\nabla\left(\rho^{\varepsilon}\right)^{\delta-1} \nabla u^{\varepsilon}\right)\right\|_{L^{2}\left(\mathbb{R}^{3}\right)}+\left\|\nabla^{k}\left(\nabla^{2}\left(\rho^{\varepsilon}\right)^{\delta-1} u^{\varepsilon}\right)\right\|_{L^{2}\left(\mathbb{R}^{3}\right)}\right) \\
& \quad \text { for } k=0,1, \\
& \left\|\nabla^{2}\left(\left(\rho^{\varepsilon}\right)^{\delta-1} \nabla^{2} u^{\varepsilon}\right)\right\|_{L^{2}\left(\mathbb{R}^{3}\right)} \\
& \leq C \varepsilon^{-1}\left\|\left(\rho^{\varepsilon}\right)^{\delta-1} \nabla^{2}\left(\left(\rho^{\varepsilon}\right)^{1-\delta}\left(u_{t}^{\varepsilon}+u^{\varepsilon} \cdot \nabla u^{\varepsilon}\right)+\left(\rho^{\varepsilon}\right)^{-\delta} \nabla p\left(\rho^{\varepsilon}\right)\right)\right\|_{L^{2}\left(\mathbb{R}^{3}\right)} \\
& \quad+C\left\|\left(\rho^{\varepsilon}\right)^{\delta-1} \nabla^{2}\left(\nabla\left(\log \rho^{\varepsilon}\right) \nabla u^{\varepsilon}\right)\right\|_{L^{2}\left(\mathbb{R}^{3}\right)} \\
& \quad+C\left(\left\|\nabla\left(\rho^{\varepsilon}\right)^{\delta-1} \nabla^{3} u^{\varepsilon}\right\|_{L^{2}\left(\mathbb{R}^{3}\right)}+\left\|\nabla^{2}\left(\rho^{\varepsilon}\right)^{\delta-1} \nabla^{2} u^{\varepsilon}\right\|_{L^{2}\left(\mathbb{R}^{3}\right)}\right) ;
\end{aligned}
$$

- when $\delta=1$ in [36], $T^{\varepsilon}=O(\varepsilon)$, and

$$
\begin{aligned}
\left\|\nabla^{k+2} u^{\varepsilon}\right\|_{L^{2}\left(\mathbb{R}^{2}\right) \leq} & C \varepsilon^{-1}\left\|\nabla^{k}\left(u_{t}^{\varepsilon}+u^{\varepsilon} \cdot \nabla u^{\varepsilon}+2 \nabla \rho^{\varepsilon}\right)\right\|_{L^{2}\left(\mathbb{R}^{2}\right)} \\
& +C\left\|\nabla^{k}\left(\nabla\left(\log \rho^{\varepsilon}\right) \nabla u^{\varepsilon}\right)\right\|_{L^{2}\left(\mathbb{R}^{2}\right)} \quad \text { for } k=0,1,2 .
\end{aligned}
$$

These indicate that the existing frameworks do not seem to work for the vanishing viscosity limit problem. Thus, in order to study the vanishing viscosity limit of strong solutions in the whole space from the Navier-Stokes to the Euler equations for compressible flow with initial vacuum in some open set or at the far-field, new ideas are required.

As far as we know, there are only a few results on the inviscid limit problem for multidimensional compressible viscous flow with vacuum. Recently, for the case $\delta=1$ in (1.4), by using the following structure to control the behavior of the fluid velocity $u^{\varepsilon}$,

$$
u_{t}^{\varepsilon}+u^{\varepsilon} \cdot \nabla u^{\varepsilon}+\frac{2}{\gamma-1} c^{\varepsilon} \nabla c^{\varepsilon}+\varepsilon L u^{\varepsilon}=\varepsilon \nabla\left(\log \rho^{\varepsilon}\right) Q\left(u^{\varepsilon}\right),
$$

with $Q(u)=2 \alpha D(u)+\beta \operatorname{div} u \mathbb{I}_{2}$, the following uniform estimate was obtained in DingZhu ([16]):

$$
\begin{aligned}
& \sup _{0 \leq t \leq T_{*}}\left(\left\|\left(c^{\varepsilon}, u^{\varepsilon}\right)(t, \cdot)\right\|_{H^{3}\left(\mathbb{R}^{2}\right)}^{2}+\left\|\nabla \log \rho^{\varepsilon}(t, \cdot)\right\|_{H^{1}\left(\mathbb{R}^{2}\right)}^{2}+\varepsilon\left\|\nabla^{3} \log \rho^{\varepsilon}(t, \cdot)\right\|_{L^{2}\left(\mathbb{R}^{2}\right)}^{2}\right) \\
& \quad+\varepsilon \int_{0}^{T_{*}}\left\|\nabla^{4} u^{\varepsilon}(t, \cdot)\right\|_{L^{2}\left(\mathbb{R}^{2}\right)}^{2} \mathrm{~d} t \leq C_{0}
\end{aligned}
$$

for some constant $C_{0}=C_{0}\left(A, \gamma, \alpha, \beta, \rho_{0}^{\varepsilon}, u_{0}^{\varepsilon}\right)>0$ and $T_{*}>0$ independent of $\varepsilon$. Based on this fact, the corresponding inviscid limit problem for strong solutions of the shallow water equations in $\mathbb{R}^{2}$, i.e., in (1.1),

$$
\gamma=2, \quad \mu=\alpha \rho, \quad \lambda=\beta \rho,
$$

was studied under the assumption that $\rho^{\varepsilon} \rightarrow 0$ as $|x| \rightarrow \infty$. Some related results can also be found in Geng-Li-Zhu ([18]). 
In this paper we first observe a quasi-symmetric hyperbolic-singular elliptic coupled structure of system (1.1) with some singular terms of first and second orders (see (3.8)). Based on this, we prove that the inviscid flow (1.6) can be regarded as the viscous flow (1.1) with vanishing physical viscosities for the regular solutions with far-field vacuum in the sense of Definition 2.1, when the viscous stress tensor is of form (1.3)-(1.5) for the most physical case $0<\delta<1$. Our analysis is based mainly on the following structure of $u^{\varepsilon}$ :

$$
u_{t}^{\varepsilon}+u^{\varepsilon} \cdot \nabla u^{\varepsilon}+\frac{2}{\gamma-1} c^{\varepsilon} \nabla c^{\varepsilon}=-\varepsilon\left(\rho^{\varepsilon}\right)^{\delta-1} L u^{\varepsilon}+\frac{2 \delta \varepsilon}{\delta-1}\left(\rho^{\varepsilon}\right)^{\frac{\delta-1}{2}} \nabla\left(\rho^{\varepsilon}\right)^{\frac{\delta-1}{2}} Q\left(u^{\varepsilon}\right),
$$

which is a special quasi-linear parabolic system with some singular coefficients and source terms near the vacuum state. However, compared with the structure of equations (1.18) which controls the behavior of $u^{\varepsilon}$ for the case $\delta=1$ in [16], in order to make sure that the estimates and the life span of the solutions are independent of $\varepsilon$, several new difficulties arise:

(i) The source term contains a strong singularity, since

$$
2\left(\rho^{\varepsilon}\right)^{\frac{\delta-1}{2}} \nabla\left(\rho^{\varepsilon}\right)^{\frac{\delta-1}{2}} Q\left(u^{\varepsilon}\right)=(\delta-1)\left(\rho^{\varepsilon}\right)^{\delta-1} \nabla\left(\log \rho^{\varepsilon}\right) Q\left(u^{\varepsilon}\right),
$$

whose behavior becomes more singular than that of $\nabla\left(\log \rho^{\varepsilon}\right) Q\left(u^{\varepsilon}\right)$ in [16] when $\rho^{\varepsilon} \rightarrow 0$ as $|x| \rightarrow \infty$, since $\delta<1$. In fact, the time evolution of $\nabla\left(\rho^{\varepsilon}\right)^{\frac{\delta-1}{2}}$ can be controlled by a symmetric hyperbolic system with a second-order singular term $\left(\rho^{\varepsilon}\right)^{\frac{\delta-1}{2}} \nabla \operatorname{div} u^{\varepsilon}$ (see (3.7)). This does not appear in the uniform estimates on $\nabla \log \rho^{\varepsilon}$ for the shallow water equations in [16].

(ii) The coefficient $\left(\rho^{\varepsilon}\right)^{\delta-1}$ in front of the Lamé operator $L$ tends to $\infty$ when $\rho^{\varepsilon} \rightarrow$ 0 , rather than 1 as in [16] (see (1.18)-(1.19)), as $|x| \rightarrow \infty$. Then we need to pay additional attention to make sure that $\left(\rho^{\varepsilon}\right)^{\delta-1} L u^{\varepsilon}$ is well defined in some weighted functional space.

We believe that the methodology developed in this paper will also provide a better understanding for other related vacuum problems for the degenerate viscous flow in a more general framework, such as the inviscid limit problem for multidimensional entropy weak solutions in the whole space.

The rest of this paper is divided into seven sections. In Section 2 we first introduce the notion of regular solutions of the Cauchy problem for the compressible Navier-Stokes equations (1.1) with far-field vacuum and state our main results.

In Section 3 we reformulate the highly degenerate equations (1.1) into a trackable system (see (3.8) below), which consists of a symmetric hyperbolic system for $\nabla\left(\rho^{\varepsilon}\right)^{\frac{\delta-1}{2}}$ but with a possible singular second-order term of the fluid velocity, $\frac{\delta-1}{2}\left(\rho^{\varepsilon}\right)^{\frac{\delta-1}{2}} \nabla \operatorname{div} u^{\varepsilon}$, and a quasi-symmetric hyperbolic-singular elliptic coupled system for $\left(c^{\varepsilon}, u^{\varepsilon}\right)$, which contains some possible singular terms of first order, such as $\left(\rho^{\varepsilon}\right)^{\frac{\delta-1}{2}} \nabla\left(\rho^{\varepsilon}\right)^{\frac{\delta-1}{2}} \cdot \nabla u^{\varepsilon}$.

In Section 4 we consider the well-posedness with far-field vacuum of the corresponding Cauchy problem of the reformulated system (3.8) through an elaborate linearization 
and approximation process, whose life span is uniformly positive with respect to $\varepsilon$. Moreover, we obtain some uniform estimates of $\left(c^{\varepsilon}, u^{\varepsilon}\right)$ in $H^{3}\left(\mathbb{R}^{3}\right)$ that are independent of $\varepsilon$. Denote $h^{\varepsilon}:=(A \gamma)^{\frac{1-\delta}{2(\gamma-1)}}\left(c^{\varepsilon}\right)^{\frac{\delta-1}{\gamma-1}}$. These estimates are achieved by the following five steps:

1. In Section 4.1, a uniform elliptic operator

$$
\frac{(\gamma-1)^{2}}{4}\left(\left(h^{\varepsilon}\right)^{2}+v^{2}\right) L u^{\varepsilon}
$$

with artificial viscosity coefficients is added to the momentum equations for sufficiently small constant $v>0$ so that the global well-posedness of the approximate solutions of the corresponding linearized problem (4.4) is established for $\left(h^{\varepsilon}, c^{\varepsilon}, u^{\varepsilon}\right)$ with initial data

$$
\left.\left(h^{\varepsilon}, c^{\varepsilon}, u^{\varepsilon}\right)\right|_{t=0}=\left(h_{0}^{\varepsilon}, c_{0}^{\varepsilon}, u_{0}^{\varepsilon}\right)(x)=\left((A \gamma)^{-\frac{\iota}{2}}\left(c_{0}^{\varepsilon}+\eta\right)^{\iota}, c_{0}^{\varepsilon}, u_{0}^{\varepsilon}\right)(x),
$$

where $c_{0}^{\varepsilon}=c^{\varepsilon}(0, x)=\sqrt{A \gamma}\left(\rho_{0}^{\varepsilon}\right)^{\frac{\gamma-1}{2}}, \iota=\frac{\delta-1}{\gamma-1}<0$, and $\eta \in(0,1]$ is some constant.

2. In Section 4.2 , we obtain the uniform estimates of $\left(c^{\varepsilon}, u^{\varepsilon}\right)$ in $H^{3}$ with respect to $(v, \eta, \varepsilon)$ for the linearized problem (4.4).

3. In Section 4.3, the approximate solutions of the Cauchy problem of the reformulated nonlinear system (4.32) are established by an iteration scheme and the conclusions obtained in Sections 4.1 and 4.2, whose life spans are uniformly positive with respect to $(v, \eta, \varepsilon)$.

4. In Section 4.4, based on the conclusions of Section 4.3, we recover the solution of the nonlinear reformulated problem (4.56) without artificial viscosity by passing to the limit as $v \rightarrow 0$.

5. In Section 4.5, based on the conclusions of Section 4.4, we recover the solution of the nonlinear reformulated problem (3.8)-(3.10) allowing the vacuum state in the far-field by passing to the limit as $\eta \rightarrow 0$.

Then, in Section 5, we show that the uniform energy estimates of the reformulated problem obtained in Section 4 indeed imply the desired uniform energy and life span estimates of the original problem. In Section 6 we establish the vanishing viscosity limit from the degenerate viscous flow to the inviscid flow with far-field vacuum. Section 7 is devoted to a nonexistence theory for global regular solutions with $L^{\infty}$ decay of $u^{\varepsilon}$. In the appendix, we list some basic lemmas that are used in our proof. It is worth pointing out that our framework in this paper can be applied to other physical dimensions, say 1 and 2, via some minor modifications.

\section{Main theorems}

In this section we state our main results. Throughout this paper from now on, we adopt the following simplified notation; most of it is for the standard homogeneous and 
inhomogeneous Sobolev spaces:

$$
\begin{aligned}
& \|f\|_{s}=\|f\|_{H^{s}\left(\mathbb{R}^{3}\right)}, \quad|f|_{q}=\|f\|_{L^{q}\left(\mathbb{R}^{3}\right)}, \quad\|f\|_{m, q}=\|f\|_{W^{m, q}\left(\mathbb{R}^{3}\right)}, \\
& |f|_{C^{k}}=\|f\|_{C^{k}\left(\mathbb{R}^{3}\right)}, \quad\|f\|_{X Y(t)}=\|f\|_{X\left([0, t] ; Y\left(\mathbb{R}^{3}\right)\right)}, \\
& \|(f, g)\|_{X}=\|f\|_{X}+\|g\|_{X}, \quad X([0, T] ; Y)=X\left([0, T] ; Y\left(\mathbb{R}^{3}\right)\right), \\
& D^{k, r}=\left\{f \in L_{\mathrm{loc}}^{1}\left(\mathbb{R}^{3}\right):|f|_{D^{k, r}}=\left|\nabla^{k} f\right|_{r}<\infty\right\} \\
& D^{k}=D^{k, 2}, \quad D^{1}=\left\{f \in L^{6}\left(\mathbb{R}^{3}\right):|f|_{D^{1}}=|\nabla f|_{2}<\infty\right\} \\
& |f|_{D^{1}}=\|f\|_{D^{1}\left(\mathbb{R}^{3}\right)}, \quad\|f\|_{X_{1} \cap X_{2}}=\|f\|_{X_{1}}+\|f\|_{X_{2}} .
\end{aligned}
$$

A detailed description of the homogeneous Sobolev spaces can be found in Galdi ([17]).

Now we introduce a proper class of solutions, called regular solutions, of the Cauchy problem (1.1)-(1.5) with (1.7)-(1.8).

Definition 2.1. Let $T>0$. A solution $\left(\rho^{\varepsilon}, u^{\varepsilon}\right)$ of the Cauchy problem (1.1)-(1.5) with (1.7)-(1.8) is called a regular solution in $[0, T] \times \mathbb{R}^{3}$ if $\left(\rho^{\varepsilon}, u^{\varepsilon}\right)$ is a weak solution in the sense of distributions and satisfies the following regularity properties:

(i) $\quad \rho^{\varepsilon}>0, \quad c^{\varepsilon} \in C\left([0, T] ; H^{3}\right), \quad \nabla\left(\rho^{\varepsilon}\right)^{\frac{\delta-1}{2}} \in C\left([0, T] ; L^{\infty} \cap D^{2}\right)$;

(ii) $\quad u^{\varepsilon} \in C\left([0, T] ; H_{\mathrm{loc}}^{3}\right) \cap L^{\infty}\left([0, T] ; H^{3}\right), \quad\left(\rho^{\varepsilon}\right)^{\frac{1-\delta}{2}} u^{\varepsilon} \in C\left([0, T] ; H^{3}\right)$, $\left(\rho^{\varepsilon}\right)^{\frac{\delta-1}{2}} \nabla u^{\varepsilon} \in L^{2}\left([0, T] ; H^{3}\right), \quad\left(\rho^{\varepsilon}\right)^{\frac{1-\delta}{2}} u_{t}^{\varepsilon} \in L^{\infty}\left([0, T] ; H^{1}\right) \cap L^{2}\left([0, T] ; H^{2}\right)$.

Remark 2.1. It follows from Definition 2.1 and the Gagliardo-Nirenberg inequality that $\nabla\left(\rho^{\varepsilon}\right)^{\frac{\delta-1}{2}} \in L^{\infty}$, which means that the vacuum occurs only in the far-field. According to the analysis of the structure of system (1.1) shown in Section 3 below, the regular solutions defined above not only select the velocity in a physically reasonable way when the density approaches zero in the far-field (see Remark 7.1), but also make the problem trackable through an elaborate linearization and approximation process (see Section 4).

Now we are ready to state our first result on the existence and uniform estimates with respect to $\varepsilon$. Denote the total mass and the total energy in $\mathbb{R}^{3}$ respectively as

$$
m(t):=\int \rho^{\varepsilon}(t, x) \mathrm{d} x, \quad E(t):=\int\left(\frac{1}{2} \rho^{\varepsilon}\left|u^{\varepsilon}\right|^{2}+\frac{A\left(\rho^{\varepsilon}\right)^{\gamma}}{\gamma-1}\right)(t, x) \mathrm{d} x .
$$

Theorem 2.1 (Existence and uniform estimates). Let $\varepsilon \in(0,1]$, and let the physical parameters $(\gamma, \delta, \alpha, \beta)$ satisfy

$$
\gamma>1, \quad 0<\delta<1, \quad \alpha>0, \quad \alpha+\beta \geq 0 .
$$

Let the initial data $\left(\rho_{0}^{\varepsilon}, u_{0}^{\varepsilon}\right)$ satisfy

$$
\rho_{0}^{\varepsilon}>0, \quad\left(c_{0}^{\varepsilon}, u_{0}^{\varepsilon}\right) \in H^{3}, \quad \varepsilon^{\frac{1}{2}} \nabla\left(\rho_{0}^{\varepsilon}\right)^{\frac{\delta-1}{2}} \in D^{1} \cap D^{2}, \quad \varepsilon^{\frac{1}{4}} \nabla\left(\rho_{0}^{\varepsilon}\right)^{\frac{\delta-1}{4}} \in L^{4},
$$

and the quantity

$$
\mathcal{E}_{0}=\left\|\left(c_{0}^{\varepsilon}, u_{0}^{\varepsilon}\right)\right\|_{3}+\varepsilon^{\frac{1}{2}}\left\|\nabla\left(\rho_{0}^{\varepsilon}\right)^{\frac{\delta-1}{2}}\right\|_{D^{1} \cap D^{2}}+\varepsilon^{\frac{1}{4}}\left|\nabla\left(\rho_{0}^{\varepsilon}\right)^{\frac{\delta-1}{4}}\right|_{4}
$$


be uniformly bounded with respect to $\varepsilon$. Then there exist $T_{*}>0$ and $C_{0}>0$ independent of $\varepsilon$ such that there exists a unique regular solution $\left(\rho^{\varepsilon}, u^{\varepsilon}\right)$ of the Cauchy problem (1.1)(1.5) with (1.7)-(1.8) in $\left[0, T_{*}\right] \times \mathbb{R}^{3}$ so that the following estimates hold for all $t \in\left[0, T_{*}\right]$ :

$$
\begin{aligned}
& \sup _{0 \leq t \leq T_{*}}\left(\left\|c^{\varepsilon}(t, \cdot)\right\|_{3}^{2}+\varepsilon\left\|\nabla\left(\rho^{\varepsilon}\right)^{\frac{\delta-1}{2}}(t, \cdot)\right\|_{D^{1} \cap D^{2}}^{2}+\text { ess } \sup _{0 \leq t \leq T_{*}}\left\|u^{\varepsilon}(t, \cdot)\right\|_{3}^{2}\right. \\
& \quad+\varepsilon \int_{0}^{T_{*}} \sum_{i=1}^{4}\left|\left(\rho^{\varepsilon}\right)^{\frac{\delta-1}{2}} \nabla^{i} u^{\varepsilon}(t, \cdot)\right|_{2}^{2} \mathrm{~d} t \leq C_{0} .
\end{aligned}
$$

Moreover, if $m(0)<\infty$ is additionally assumed, then the regular solution $\left(\rho^{\varepsilon}, u^{\varepsilon}\right)$ obtained above has finite total mass and total energy:

$m(t)=m(0)=\int \rho_{0}^{\varepsilon}(x) \mathrm{d} x<\infty, \quad E(t) \leq E(0)=\int\left(\frac{1}{2} \rho_{0}^{\varepsilon}\left|u_{0}^{\varepsilon}\right|^{2}+\frac{P\left(\rho_{0}^{\varepsilon}\right)}{\gamma-1}\right) \mathrm{d} x<\infty$ for $0 \leq t \leq T_{*}$.

Remark 2.2. Regarding the above initial assumption (2.2), we remark that (2.2) identifies a class of admissible initial data that provide the unique solvability of problem (1.1)-(1.5) with (1.7)-(1.8). For example,

$$
\rho_{0}^{\varepsilon}(x)=f(x) \chi\left(\frac{x}{10}\right)+\frac{1}{1+|x|^{2 a}}, \quad u_{0}^{\varepsilon}(x) \in H^{3}\left(\mathbb{R}^{3}\right),
$$

where

$$
0 \leq f(x) \in C^{3}\left(\mathbb{R}^{3}\right), \quad \frac{3}{2(\gamma-1)}<a<\frac{1}{2(1-\delta)},
$$

and $\chi(x) \in C_{c}^{\infty}\left(\mathbb{R}^{3}\right)$ is a truncation function satisfying

$$
0 \leq \chi(x) \leq 1, \quad \chi(x)= \begin{cases}1 & \text { if }|x| \leq 1 \\ 0 & \text { if }|x| \geq 2\end{cases}
$$

Moreover, condition $\varepsilon^{\frac{1}{4}} \nabla\left(\rho_{0}^{\varepsilon}\right)^{\frac{\delta-1}{4}} \in L^{4}$ is only used in the approximation process of the initial data from non-vacuum flow to flow with a far-field vacuum (see (4.16) below), which is not used for our energy estimates. We believe that this condition could be removed if an improved approximation scheme were developed.

Now, based on the well-posedness results in Theorems 1.1 and 2.1 for both viscous and inviscid flows, we can show the following asymptotic behavior as $\varepsilon \rightarrow 0$ :

Theorem 2.2 (Inviscid limit). Let $\varepsilon \in(0,1]$ and (2.1)-(2.3) hold. If we additionally assume that there exist functions $\left(\rho_{0}(x), u_{0}(x)\right)$ defined in $\mathbb{R}^{3}$ so that

$$
\lim _{\varepsilon \rightarrow 0}\left|\left(c_{0}^{\varepsilon}-c_{0}, u_{0}^{\varepsilon}-u_{0}\right)\right|_{2}=0
$$

then there exist functions $(\rho, u)$ defined in $\left[0, T_{*}\right] \times \mathbb{R}^{3}$ such that

$$
\sup _{0 \leq t \leq T_{2}}\|(c, u)(t, \cdot)\|_{3}^{2} \leq C \quad \text { for some constant } C>0
$$


and, for any constant $s^{\prime} \in[0,3)$,

$$
\lim _{\varepsilon \rightarrow 0} \sup _{0 \leq t \leq T_{*}}\left\|\left(c^{\varepsilon}-c, u^{\varepsilon}-u\right)(t, \cdot)\right\|_{H_{\mathrm{loc}}^{s^{\prime}}\left(\mathbb{R}^{3}\right)}=0 .
$$

Furthermore, $(\rho, u)$ is the unique regular solution of the Cauchy problem (1.6) and (1.13) in Theorem 1.1.

The above theorem implies the following result:

Corollary 2.1. Let $\varepsilon \in(0,1]$ and $(2.1)-(2.3)$ hold. Suppose that $\left(\rho^{\varepsilon}, u^{\varepsilon}\right)$ is the regular solution of problem (1.1)-(1.5) with (1.7)-(1.8) in Theorem 2.1, and $(\rho, u)$ is the regular solution of (1.6) with (1.13) in Theorem 1.1. If

$$
\left.\left(\rho^{\varepsilon}, u^{\varepsilon}\right)\right|_{t=0}=\left.(\rho, u)\right|_{t=0}=\left(\rho_{0}, u_{0}\right)
$$

then there exists $T_{*}>0$ independent of $\varepsilon$ such that $\left(\rho^{\varepsilon}, u^{\varepsilon}\right)$ converges to $(\rho, u)$ in $\left[0, T_{*}\right] \times \mathbb{R}^{3}$ as $\varepsilon \rightarrow 0$ in the sense of distributions. Moreover, for any constant $s^{\prime} \in[0,3)$, we also have (2.9).

Moreover, we can also obtain the following corollary:

Corollary 2.2. Let $\delta \in(0,1)$, and $\left(\rho_{0}, u_{0}\right)$ satisfy (1.14). Then, for every $\varepsilon \in(0,1]$, there exist $\left(\rho_{0}^{\varepsilon}, u_{0}^{\varepsilon}\right)$ satisfying assumption (2.2) such that

$$
\left\|\left(c_{0}^{\varepsilon}, u_{0}^{\varepsilon}\right)\right\|_{3}^{2}+\varepsilon\left\|\nabla\left(\rho_{0}^{\varepsilon}\right)^{\frac{\delta-1}{2}}\right\|_{D^{1} \cap D^{2}}^{2}+\varepsilon^{\frac{1}{2}}\left|\nabla\left(\rho_{0}^{\varepsilon}\right)^{\frac{\delta-1}{4}}\right|_{4}^{2} \leq C_{0}
$$

for some constant $C_{0}>0$ independent of $\varepsilon$ and

$$
\lim _{\varepsilon \rightarrow 0}\left\|\left(c_{0}^{\varepsilon}-c_{0}, u_{0}^{\varepsilon}-u_{0}\right)\right\|_{3}=0 .
$$

Moreover, the corresponding Cauchy problem (1.6) and (1.13) can be regarded as a limit problem of the Cauchy problem (1.1)-(1.5) with (1.7)-(1.8) as $\varepsilon \rightarrow 0$ in the sense of (2.9).

Naturally, a further question is whether the solution obtained in Theorem 2.1 can be extended globally in time under the assumption that the initial data is a small perturbation around some background solution. Under the assumption that

$$
\rho_{0}^{\varepsilon}(x) \rightarrow \bar{\rho} \quad \text { as }|x| \rightarrow 0
$$

for some constant $\bar{\rho}>0$, the classical theories, no matter whether for constant viscous flow (e.g., [26, 39, 44]) or degenerate viscous flow (e.g., [30]) away from a vacuum, all indicate that the corresponding background solution for $\left(\rho^{\varepsilon}, u^{\varepsilon}\right)$ must be $(\bar{\rho}, 0)$ with the following large-time behavior:

$$
\limsup _{t \rightarrow \infty}\left|u^{\varepsilon}(t, \cdot)\right|_{\infty}=0 .
$$

However, when a vacuum appears, the situation for degenerate viscous flow is somewhat surprising, since such an extension seems impossible when the initial momentum is nonzero. More precisely, denote the flow momentum by

$$
\mathbb{P}(t):=\int\left(\rho^{\varepsilon} u^{\varepsilon}\right)(t, x) \mathrm{d} x .
$$


Theorem 2.3. Assume that $0<m(0)<\infty,|\mathbb{P}(0)|>0, \varepsilon \geq 0$, and (2.1) hold. Then there is no global regular solution $\left(\rho^{\varepsilon}, u^{\varepsilon}\right)$ in the sense of Theorem 1.1 or 2.1 satisfying (2.11).

Finally, we show that the condition $\varepsilon^{\frac{1}{2}} \nabla\left(\rho_{0}^{\varepsilon}\right)^{\frac{\delta-1}{2}} \in D^{1}$ in (2.2) can be replaced by other conditions such as $\varepsilon^{\frac{1}{2}} \nabla\left(\rho_{0}^{\varepsilon}\right)^{\frac{\delta-1}{2}} \in L^{q} \cap D^{1,3}$ for any constant $q>3$ :

Theorem 2.4. Let $\varepsilon \in(0,1]$ and $(2.1)$ hold. Let $q \in(3, \infty]$ be a fixed constant. Assume that the initial data $\left(\rho_{0}^{\varepsilon}, u_{0}^{\varepsilon}\right)$ satisfy

$$
\rho_{0}^{\varepsilon}>0, \quad\left(c_{0}^{\varepsilon}, u_{0}^{\varepsilon}\right) \in H^{3}, \quad \varepsilon^{\frac{1}{2}} \nabla\left(\rho_{0}^{\varepsilon}\right)^{\frac{\delta-1}{2}} \in L^{q} \cap D^{1,3} \cap D^{2}, \quad \varepsilon^{\frac{1}{4}} \nabla\left(\rho_{0}^{\varepsilon}\right)^{\frac{\delta-1}{4}} \in L^{6},
$$

and

$$
\left\|\left(c_{0}^{\varepsilon}, u_{0}^{\varepsilon}\right)\right\|_{3}+\varepsilon^{\frac{1}{2}}\left\|\nabla\left(\rho_{0}^{\varepsilon}\right)^{\frac{\delta-1}{2}}\right\|_{L^{q} \cap D^{1,3} \cap D^{2}}+\varepsilon^{\frac{1}{4}}\left|\nabla\left(\rho_{0}^{\varepsilon}\right)^{\frac{\delta-1}{4}}\right|_{6}
$$

is uniformly bounded with respect to $\varepsilon$. Then there exist $T_{*}>0$ and $C>0$, both independent of $\varepsilon$, such that the unique regular solution $\left(\rho^{\varepsilon}, u^{\varepsilon}\right)$ of the Cauchy problem (1.1)-(1.5) with (1.7)-(1.8) exists in $\left[0, T_{*}\right] \times \mathbb{R}^{3}$ with the following estimates:

$$
\begin{aligned}
& \sup _{0 \leq t \leq T_{*}}\left(\left\|c^{\varepsilon}(t, \cdot)\right\|_{3}^{2}+\varepsilon\left\|\nabla\left(\rho^{\varepsilon}\right)^{\frac{\delta-1}{2}}(t, \cdot)\right\|_{L^{q} \cap D^{1,3} \cap D^{2}}^{2}+\text { ess } \sup _{0 \leq t \leq T_{*}}\left\|u^{\varepsilon}(t, \cdot)\right\|_{3}^{2}\right. \\
& \quad+\varepsilon \int_{0}^{T_{*}} \sum_{i=1}^{4}\left|\left(\rho^{\varepsilon}\right)^{\frac{\delta-1}{2}} \nabla^{i} u^{\varepsilon}(t, \cdot)\right|_{2}^{2} \mathrm{~d} t \leq C_{0}
\end{aligned}
$$

Moreover, under proper changes to the corresponding assumptions, the results obtained in Theorems 2.1-2.3 and Corollaries 2.1-2.2 still hold.

The proof of this theorem is similar to those of Theorems 2.1-2.3 and Corollaries 2.1-2.2. Thus, we omit its details.

Remark 2.3. The conditions in (2.12) identify a class of admissible initial data that provide the unique solvability of problem (1.1)-(1.5) with (1.7)-(1.8) such as the one shown in (2.5)-(2.6) with

$$
0 \leq f(x) \in C^{3}\left(\mathbb{R}^{3}\right), \quad \frac{3}{2(\gamma-1)}<a<\frac{1-3 / q}{1-\delta} .
$$

We remark that our framework in this paper is applicable to other physical dimensions, say 1 and 2, via some minor modifications.

\section{Reformulation}

In this section we reformulate the highly degenerate equations (1.1) into a trackable system that consists of a symmetric hyperbolic system with a possibly singular second-order term of the fluid velocity, and a quasi-symmetric hyperbolic-singular elliptic coupled system. For simplicity, throughout this section we denote $\left(\rho^{\varepsilon}, u^{\varepsilon}, c^{\varepsilon}, \psi^{\varepsilon}, h^{\varepsilon}\right)$ by $(\rho, u, c, \psi, h)$ and $\left(\rho_{0}^{\varepsilon}, u_{0}^{\varepsilon}, c_{0}^{\varepsilon}, \psi_{0}^{\varepsilon}, h_{0}^{\varepsilon}\right)$ by $\left(\rho_{0}, u_{0}, c_{0}, \psi_{0}, h_{0}\right)$, respectively. 


\subsection{New variables}

Let $T>0$ be a fixed finite time. For $\delta \in(0,1)$, when $\rho(t, x)>0$ for $(t, x) \in[0, T] \times \mathbb{R}^{3}$, $(1.1)_{2}$ can be formally rewritten as

$$
u_{t}+u \cdot \nabla u+\frac{2 A \gamma}{\gamma-1} \rho^{\frac{\gamma-1}{2}} \nabla \rho^{\frac{\gamma-1}{2}}+\varepsilon \rho^{\delta-1} L u=\frac{2 \delta \varepsilon}{\delta-1} \rho^{\frac{\delta-1}{2}} \nabla \rho^{\frac{\delta-1}{2}} Q(u),
$$

where $\rho^{\frac{\gamma-1}{2}}$ is a constant multiple of the local sound speed $c=\sqrt{p^{\prime}(\rho)}$. Then, in order to govern velocity $u$ via the above quasi-linear parabolic equations with far-field vacuum,

(i) it is necessary to control the behavior of the special source term

$$
\frac{2 \delta \varepsilon}{\delta-1} \rho^{\frac{\delta-1}{2}} \nabla \rho^{\frac{\delta-1}{2}} Q(u)
$$

since $\delta-1<0$ and

$$
\rho(t, x) \rightarrow 0 \quad \text { as }|x| \rightarrow \infty \text { for } t \in[0, T] ;
$$

(ii) note that the coefficient $\rho^{\delta-1}$ in front of the Lamé operator $L$ tends to $\infty$ as $\rho \rightarrow 0$ in the far-field, so that it is necessary to show that $\rho^{\delta-1} L u$ is well defined at least in the space of continuous functions, in order that the solution obtained is regular when $t>0$.

Therefore, the three quantities

$$
\rho^{\frac{\gamma-1}{2}}, \quad \nabla \rho^{\frac{\delta-1}{2}}, \quad \rho^{\delta-1} L u
$$

play a significant role in our analysis on the regularity of the fluid velocity $u$. In fact, in terms of the fluid velocity $u$, and

$$
c=\sqrt{A \gamma} \rho^{\frac{\gamma-1}{2}}, \quad \psi=\nabla \rho^{\frac{\delta-1}{2}}=\nabla h=\left(\psi^{(1)}, \psi^{(2)}, \psi^{(3)}\right)
$$

system (1.1) can be rewritten as the following enlarged system:

$$
\left\{\begin{array}{l}
\psi_{t}+\nabla(u \cdot \psi)+\frac{\delta-1}{2} \psi \operatorname{div} u+\frac{\delta-1}{2} h \nabla \operatorname{div} u=0, \\
c_{t}+u \cdot \nabla c+\frac{\gamma-1}{2} c \operatorname{div} u=0 \\
u_{t}+u \cdot \nabla u+\frac{2}{\gamma-1} c \nabla c+\varepsilon h^{2} L u=\frac{2 \delta \varepsilon}{\delta-1} h \psi Q(u),
\end{array}\right.
$$

where

$$
h=\rho^{\frac{\delta-1}{2}}=(A \gamma)^{-\frac{\iota}{2}} c^{\iota}, \quad \iota=\frac{\delta-1}{\gamma-1}<0 .
$$

The initial data are given by

$$
\left.(\psi, c, u)\right|_{t=0}=\left(\psi_{0}, c_{0}, u_{0}\right)(x):=\left(\nabla \rho_{0}^{\frac{\delta-1}{2}}, \sqrt{A \gamma} \rho_{0}^{\frac{\gamma-1}{2}}, u_{0}\right)(x) \text { for } x \in \mathbb{R}^{3},
$$

so that

$$
\left(\psi_{0}, c_{0}, u_{0}\right) \rightarrow(0,0,0) \text { as }|x| \rightarrow \infty
$$




\subsection{Mathematical structure of the reformulated system}

Now we introduce the desired quasi-symmetric hyperbolic-singular elliptic coupled structure in order to deal with the corresponding inviscid limit problem.

The new system (3.3) still seems un-trackable for the purpose of constructing the regular solutions with far-field vacuum in $H^{3}$ under the following initial assumption:

$$
c_{0}>0, \quad\left(c_{0}, u_{0}\right) \in H^{3}, \quad \psi_{0} \in D^{1} \cap D^{2} .
$$

First, even if $h \nabla \operatorname{div} u$ could be controlled by the singular elliptic operator $h^{2} L u$ appearing in the momentum equations, the special source term $\psi$ cannot be controlled by a scalar transport equation, but by a quasi-linear hyperbolic system. It follows from the definition of $\psi$ that, if $\psi \in D^{1} \cap D^{2}$, then $\partial_{i} \psi^{(j)}=\partial_{j} \psi^{(i)}$ in the sense of distributions for $i, j=1,2,3$. Thus, (3.3) 1 can be rewritten as

$$
\psi_{t}+\sum_{l=1}^{3} B_{l} \partial_{l} \psi+B \psi+\frac{\delta-1}{2} h \nabla \operatorname{div} u=0
$$

where $B_{l}=\left(b_{i j}^{l}\right)_{3 \times 3}$, for $i, j, l=1,2,3$, are symmetric with

$$
b_{i j}^{l}=u^{(l)} \text { for } i=j, \quad b_{i j}^{l}=0 \text { otherwise, }
$$

and $B=(\nabla u)^{\top}+\frac{\delta-1}{2} \operatorname{div} u \mathbb{I}_{3}$. This indicates that the subtle source term $\psi$ could actually be controlled by the symmetric hyperbolic system with one possible singular source term $\frac{\delta-1}{2} h \nabla \operatorname{div} u$ near the vacuum.

Second, in order to make sure that the life span and the corresponding energy estimates of the regular solutions that we will obtain are uniform with respect to $\varepsilon$, we need to introduce more symmetrization arguments, except the above structure for $\psi$. In fact, letting $U=(c, u)$, according to (3.7), we rewrite the Cauchy problem (3.3)-(3.5) as

$$
\left\{\begin{array}{l}
\underbrace{\psi_{t}+\sum_{l=1}^{3} B_{l}(u) \partial_{l} \psi}_{\text {symmetric hyperbolic }}+\underbrace{B(u) \psi}_{\text {first order }}+\underbrace{\frac{\delta-1}{2}(A \gamma)^{-\frac{\iota}{2}} c^{\iota} \nabla \operatorname{div} u}_{\text {singular second order }}=0 \\
\underbrace{A_{0} U_{t}+\sum_{j=1}^{3} A_{j}(U) \partial_{j} U}_{\text {symmetric hyperbolic }}=\underbrace{-\varepsilon F(U)}_{\text {singular elliptic }}+\underbrace{\varepsilon G(\psi, U)}_{\text {singular first order }}
\end{array}\right.
$$

with the following initial data:

$$
\left.(\psi, c, u)\right|_{t=0}=\left(\psi_{0}, c_{0}, u_{0}\right)(x)=\left(\nabla \rho_{0}^{\frac{\delta-1}{2}}, \sqrt{A \gamma} \rho_{0}^{\frac{\gamma-1}{2}}, u_{0}\right)(x) \text { for } x \in \mathbb{R}^{3},
$$

so that

$$
\left(\psi_{0}, c_{0}, u_{0}\right) \rightarrow(0,0,0) \text { as }|x| \rightarrow \infty
$$


where $\partial_{l} \psi=\partial_{x_{l}} \psi, \partial_{j} U=\partial_{x_{j}} U, i, j, l=1,2,3$,

$$
\begin{gathered}
A_{0}=\left(\begin{array}{cc}
1 & 0 \\
0 & a_{1} \mathbb{I}_{3}
\end{array}\right), \quad A_{j}=\left(\begin{array}{cc}
u_{j} & \frac{\gamma-1}{2} c e_{j} \\
\frac{\gamma-1}{2} c e_{j}^{\top} & a_{1} u_{j} \mathbb{I}_{3}
\end{array}\right), \quad j=1,2,3, \\
F(U)=a_{1}\left(\begin{array}{c}
0 \\
(A \gamma)^{-\imath} c^{2 \iota} L u
\end{array}\right), \quad G(\psi, U)=a_{1}\left(\begin{array}{c}
0 \\
\frac{2 \delta}{\delta-1}(A \gamma)^{-\frac{l}{2}} c^{\iota} \psi Q(u)
\end{array}\right),
\end{gathered}
$$

with $a_{1} \equiv: \frac{(\gamma-1)^{2}}{4}, e_{1}=(1,0,0), e_{2}=(0,1,0)$, and $e_{3}=(0,0,1)$.

Remark 3.1. The hyperbolic operators $H=\left(H^{1}, H^{2}\right)$ for $(\psi, U)$,

$$
\begin{aligned}
& H^{1}(\psi):=\psi_{t}+\nabla(u \cdot \psi), \\
& H^{2}(U):=H^{2}(c, u)=\left(\begin{array}{c}
c_{t}+u \cdot \nabla c+\frac{\gamma-1}{2} c \operatorname{div} u \\
u_{t}+u \cdot \nabla u+\frac{2}{\gamma-1} c \nabla c
\end{array}\right)
\end{aligned}
$$

can be rewritten in the following symmetric hyperbolic forms:

$$
\psi_{t}+\sum_{l=1}^{3} B_{l} \partial_{l} \psi+(\nabla u)^{\top} \psi, \quad A_{0} U_{t}+\sum_{j=1}^{3} A_{j}(U) \partial_{j} U
$$

which make the $H^{3}$ estimates of $(c, u)$ possibly independent of $\varepsilon$. However, not every first-order term in system (3.8) has been written in the symmetric structure. This is the reason why we only say that system (3.8) satisfies the quasi-symmetric structure, rather than the symmetric one. Hence, new treatments are needed for the possibly singular source terms $\frac{\delta-1}{2} h \nabla \operatorname{div} u$ and $\varepsilon G(\psi, U)$.

\section{Uniform energy estimates for the reformulated problem}

This section is devoted to the establishment of uniform local-in-time well-posedness (with respect to $\varepsilon$ ) of strong solutions with far-field vacuum of the reformulated Cauchy problem (3.8)-(3.10). Moreover, some uniform estimates of $\left(c^{\varepsilon}, u^{\varepsilon}\right)$ in $H^{3}$ with respect to $\varepsilon$ can also be established. For simplicity, in this section we denote $\left(\rho^{\varepsilon}, u^{\varepsilon}, c^{\varepsilon}, \psi^{\varepsilon}, h^{\varepsilon}\right)$ by $(\rho, u, c, \psi, h)$, and $\left(\rho_{0}^{\varepsilon}, u_{0}^{\varepsilon}, c_{0}^{\varepsilon}, \psi_{0}^{\varepsilon}, h_{0}^{\varepsilon}\right)$ by $\left(\rho_{0}, u_{0}, c_{0}, \psi_{0}, h_{0}\right)$.

We first give the definition of strong solutions of the Cauchy problem (3.8)-(3.10).

Definition 4.1. Let $T>0$. A vector function $(\psi, c, u)$ is called a strong solution in $[0, T] \times \mathbb{R}^{3}$ if $(\psi, c, u)$ is a weak solution of the Cauchy problem (3.8)-(3.10) in $[0, T] \times \mathbb{R}^{3}$ in the sense of distributions, all derivatives involved in (3.8) are regular distributions, and (3.8) holds almost everywhere in $[0, T] \times \mathbb{R}^{3}$.

We now state the main result in this section on the well-posedness of the Cauchy problem for the reformulated system (3.8). 
Theorem 4.1. Let (2.1) hold and $\varepsilon \in(0,1]$. If the initial data $\left(\psi_{0}, c_{0}, u_{0}\right)$ satisfy

$$
c_{0}>0, \quad\left(c_{0}, u_{0}\right) \in H^{3}, \quad \varepsilon^{\frac{1}{2}} \psi_{0}=\varepsilon^{\frac{1}{2}}(A \gamma)^{-\frac{\iota}{2}} \nabla c_{0}^{\iota} \in D^{1} \cap D^{2}, \quad \varepsilon^{\frac{1}{4}} \nabla c_{0}^{\frac{l}{2}} \in L^{4},
$$

then there exists $T_{*}>0$ independent of $\varepsilon$ such that there is a unique strong solution $(\psi, c, u)=\left((A \gamma)^{-\frac{\iota}{2}} \nabla c^{\iota}, c, u\right)$ in $\left[0, T_{*}\right] \times \mathbb{R}^{3}$ of the Cauchy problem (3.8)-(3.10) satisfying

$$
\begin{gathered}
\psi \in C\left([0, T] ; D^{1} \cap D^{2}\right), \quad c \in C\left([0, T] ; H^{3}\right), \\
u \in C\left([0, T] ; H_{\mathrm{loc}}^{3}\right) \cap L^{\infty}\left([0, T] ; H^{3}\right), \quad c^{\iota} \nabla u \in L^{2}\left([0, T] ; H^{3}\right),
\end{gathered}
$$

and the following uniform estimates:

$$
\begin{aligned}
& \sup _{0 \leq t \leq T_{*}}\left(\|c(t, \cdot)\|_{3}^{2}+\varepsilon\|\psi(t, \cdot)\|_{D^{1} \cap D^{2}}^{2}\right)+\text { ess } \sup _{0 \leq t \leq T_{*}}\|u(t, \cdot)\|_{3}^{2} \\
& \quad+\varepsilon \int_{0}^{T_{*}} \sum_{i=1}^{4}\left|c^{\iota} \nabla^{i} u(t, \cdot)\right|_{2}^{2} \mathrm{~d} t \leq C
\end{aligned}
$$

for some positive constant $C=C\left(\alpha, \beta, A, \gamma, \delta, c_{0}, \psi_{0}, u_{0}\right)$ that is independent of $\varepsilon$.

We now prove this theorem in subsequent Sections 4.1-4.5.

\subsection{Linearization with one artificial viscosity}

In order to proceed with the nonlinear problem (3.8)-(3.10), we now consider the following linearized problem:

$$
\left\{\begin{array}{l}
h_{t}+v \cdot \nabla h+\frac{\delta-1}{2} g \operatorname{div} v=0, \\
A_{0} U_{t}+\sum_{j=1}^{3} A_{j}(V) \partial_{j} U=-\varepsilon F(v, h, u)+\varepsilon G(h, \nabla h, u), \\
\left.(h, c, u)\right|_{t=0}=\left(h_{0}, c_{0}, u_{0}\right)(x)=\left((A \gamma)^{-\frac{\iota}{2}}\left(c_{0}+\eta\right)^{\iota}, c_{0}, u_{0}\right)(x) \text { for } x \in \mathbb{R}^{3},
\end{array}\right.
$$

with

$$
\left(h_{0}, c_{0}, u_{0}\right)(x) \rightarrow\left((A \gamma)^{-\frac{\iota}{2}} \eta^{\iota}, 0,0\right) \text { as }|x| \rightarrow \infty,
$$

where $(v, \eta, \varepsilon) \in(0,1] \times(0,1] \times(0,1]$ are all positive constants,

$$
F(v, h, u):=a_{1}\left(\begin{array}{c}
0 \\
\left(h^{2}+v^{2}\right) L u
\end{array}\right), \quad G(h, \nabla h, u):=a_{1}\left(\begin{array}{c}
0 \\
\frac{2 \delta}{\delta-1} h \nabla h Q(u)
\end{array}\right),
$$

$V=(\varphi, v)$ with $\varphi$ a given function and $v=\left(v^{(1)}, v^{(2)}, v^{(3)}\right) \in \mathbb{R}^{3}$ a given vector, and $g$ is a given function satisfying

$$
\begin{gathered}
(\varphi, v, g)(0, x)=\left(c_{0}, u_{0}, h_{0}\right)(x)=\left(c_{0}, u_{0},(A \gamma)^{-\frac{\iota}{2}}\left(c_{0}+\eta\right)^{\iota}\right)(x), \\
\varphi \in C\left([0, T] ; H^{3}\right), \quad \varphi_{t} \in C\left([0, T] ; H^{2}\right), \\
g \in L^{\infty} \cap C\left([0, T] \times \mathbb{R}^{3}\right), \quad \nabla g \in C\left([0, T] ; H^{2}\right), \\
v \in C\left([0, T] ; H^{3}\right) \cap L^{2}\left([0, T] ; H^{4}\right), \\
v_{t} \in C\left([0, T] ; H^{1}\right) \cap L^{2}\left([0, T] ; H^{2}\right) \quad \text { for any } T>0 .
\end{gathered}
$$


Note that, due to the complicated structure of system (3.8) near the vacuum, the linear scheme (4.4) is carefully chosen such that this linear problem can be solved globally in time, and the desired uniform estimates of the solutions can be established. According to the analysis in Section 3, we first hope to keep the symmetric hyperbolic forms shown in (3.12) which, in our desired linear scheme, are expected to be

$$
\psi_{t}+\sum_{l=1}^{3} B_{l}(v) \partial_{l} \psi, \quad A_{0} U_{t}+\sum_{j=1}^{3} A_{j}(V) \partial_{j} U
$$

for $\psi:=\nabla h$.

Next, in order to ensure the global well-posedness of $\psi$ and the desired estimates in some positive time, the singular source term $\frac{\delta-1}{2}(A \gamma)^{-\frac{\iota}{2}} c^{\iota} \nabla \operatorname{div} u$ in (3.8) ${ }_{1}$ should be handled carefully. Possible ways to linearize this product term are

$$
\frac{\delta-1}{2} g \nabla \operatorname{div} u, \quad \text { or } \quad \frac{\delta-1}{2}(A \gamma)^{-\frac{\iota}{2}} c^{\iota} \nabla \operatorname{div} v, \quad \text { or } \quad \frac{\delta-1}{2} g \nabla \operatorname{div} v,
$$

where $V$ and $g$ satisfy assumption (4.7). For either of the first two choices, the estimates of $\psi$ in $D^{1} \cap D^{2}$ will depend on the upper bound of $g$ or $(A \gamma)^{-\frac{\iota}{2}} c^{\iota}$, i.e., the lower bound of $\rho_{0}$, which is exactly what we want to avoid. Therefore, for the linear structure of $\psi$, what we can expect should be

$$
\psi_{t}+\sum_{l=1}^{3} B_{l}(v) \partial_{l} \psi+B(v) \psi+\frac{\delta-1}{2} g \nabla \operatorname{div} v=0 .
$$

In fact, for this scheme, one can use

$$
\sum_{k=1}^{2}\left\|\nabla^{k}\left(g \nabla^{2} v\right)\right\|_{L^{2}\left([0, T] ; L^{2}\left(\mathbb{R}^{3}\right)\right)}^{2}
$$

to replace the upper bound of $g$ in the corresponding estimates, which can ensure that the desired estimates of $\psi$ are independent of the lower bound of the initial density and $\varepsilon$.

Finally, for the choice of the linearization scheme for $U$, due to the above discussion, there are at least two requirements that should be satisfied. On one hand, it needs to keep the symmetric form shown in (4.8). On the other hand, for our final aim - to approximate the nonlinear problem - the desired regularity in (4.10) for $(g, v)$ should be verified by solution $\left(c^{\iota}, u\right)$ of the linear problem, which can only be provided by the elliptic operator $c^{2 \imath} L u$. Then it seems that we should consider the following equations:

$$
A_{0} U_{t}+\sum_{j=1}^{3} A_{j}(V) \partial_{j} U=-\varepsilon F(v, h, u)+\varepsilon G(g, \psi, v),
$$

which actually is still a nonlinear system. However, even if the corresponding Cauchy problem for system (4.11) is assumed to be globally solved, we still encounter an obvious 
difficulty for considering the $L^{2}$ estimate of $u$. First, it should be pointed out that, in (4.9) and (4.11), the relationship

$$
\psi=(A \gamma)^{-\frac{\iota}{2}} \nabla c^{\iota}
$$

between $\psi$ and $c$ has been destroyed owing to term $g \nabla \operatorname{div} v$ in (4.9). Second, multiplying by $u$ on both sides of the equation for $u$ in (4.11) and integrating by parts yield

$$
\begin{aligned}
& \frac{1}{2} \frac{\mathrm{d}}{\mathrm{d} t}|u|_{2}^{2}+\varepsilon \alpha\left|(A \gamma)^{-\frac{\iota}{2}} c^{\iota} \nabla u\right|_{2}^{2}+\varepsilon(\alpha+\beta)\left|(A \gamma)^{-\frac{\iota}{2}} c^{\iota} \operatorname{div} u\right|_{2}^{2} \\
& =-\int(v \cdot \nabla u+\frac{2}{\gamma-1} \varphi \nabla c+2 \varepsilon(A \gamma)^{-\frac{\iota}{2}} c^{\iota} \underbrace{(A \gamma)^{-\frac{\iota}{2}} \nabla c^{\iota}}_{\neq \psi} Q(u)-\frac{2 \delta \varepsilon}{\delta-1} g \psi Q(v)) \cdot u \mathrm{~d} x .
\end{aligned}
$$

However, $(A \gamma)^{-\frac{\imath}{2}} \nabla c^{\iota} \neq \psi$ in this linear scheme, which means that there is no way to control term $2 \varepsilon(A \gamma)^{-\imath} c^{\iota} \nabla c^{\iota}$ in the above energy estimates. In order to overcome this difficulty, in (4.4) we first linearize the equation $h=(A \gamma)^{-\frac{\iota}{2}} c^{\iota}$ as

$$
h_{t}+v \cdot \nabla h+\frac{\delta-1}{2} g \operatorname{div} v=0,
$$

and then use $h$ to define $\psi=\nabla h$ again. The linearized equations for $u$ are chosen as

$$
u_{t}+v \cdot \nabla u+\frac{2}{\gamma-1} \varphi \nabla c+\varepsilon\left(h^{2}+v^{2}\right) L u=\frac{2 \varepsilon \delta}{\delta-1} h \psi Q(u)
$$

for any positive constant $v>0$, where the appearance of $v$ is used to compensate the lack of a lower bound for $h$. From both equation (4.12) for $h$ and the relation $\psi=\nabla h$, we can obtain the linearized equations (4.19) for $\psi$ below, which, luckily, can be shown to be still good enough to obtain the desired estimates for $\psi$ (see Lemma 4.2).

Now the global well-posedness of a classical solution of problem (4.4) in $[0, T] \times \mathbb{R}^{3}$ can be obtained by the standard theory $([13,31,41])$ at least when $(\nu, \eta, \varepsilon)$ are all positive.

Lemma 4.1. Let $T>0$ and (2.1) hold. If $\left(c_{0}, u_{0}\right)$ satisfy

$$
c_{0}>0, \quad\left(c_{0}, u_{0}\right) \in H^{3}, \quad \varepsilon^{\frac{1}{2}} \nabla c_{0}^{\iota} \in D^{1} \cap D^{2}, \quad \varepsilon^{\frac{1}{4}} \nabla c_{0}^{\frac{\iota}{2}} \in L^{4},
$$

then there exists a unique strong solution $(h, c, u)$ of problem $(4.4)$ in $[0, T] \times \mathbb{R}^{3}$ such that

$$
\begin{gathered}
h \in L^{\infty} \cap C\left([0, T] \times \mathbb{R}^{3}\right), \quad \nabla h \in C\left([0, T] ; H^{2}\right), \\
c \in C\left([0, T] ; H^{3}\right), \quad u \in C\left([0, T] ; H^{3}\right) \cap L^{2}\left([0, T] ; H^{4}\right) .
\end{gathered}
$$

We now establish the uniform energy estimates, independent of $(v, \eta, \varepsilon)$, of the unique solution $(h, U)$ of the Cauchy problem (4.4) obtained in Lemma 4.1.

\subsection{Uniform energy estimates independent of $(\nu, \eta, \varepsilon)$}

We first fix $T>0$ and a large enough positive constant $b_{0}$ (independent of $\varepsilon$ ) such that

$$
\left\|\left(c_{0}, u_{0}\right)\right\|_{3}^{2}+\varepsilon\left\|\nabla c_{0}^{\iota}\right\|_{D^{1} \cap D^{2}}^{2}+\varepsilon^{\frac{1}{2}}\left|\nabla c_{0}^{\frac{l}{2}}\right|_{4}^{2} \leq b_{0} .
$$


Then there exists $\eta_{1}>0$ such that, if $0<\eta<\eta_{1}$,

$$
\begin{array}{r}
\eta+\varepsilon^{\frac{1}{2}}\left\|\nabla\left(c_{0}+\eta\right)^{\iota}\right\|_{D^{1} \cap D^{2}}+\left|\left(c_{0}+\eta\right)^{-\iota}\right|_{\infty}+\left\|\left(c_{0}, u_{0}\right)\right\|_{3} \\
=\eta+\varepsilon^{\frac{1}{2}}\left\|\nabla h_{0}\right\|_{D^{1} \cap D^{2}}+\left|h_{0}^{-1}\right|_{\infty}+\left\|\left(c_{0}, u_{0}\right)\right\|_{3} \leq d_{0},
\end{array}
$$

where we have used the fact that $\varepsilon^{\frac{1}{4}} \nabla c_{0}^{\frac{l}{2}} \in L^{4}$, and $d_{0}>0$ is a constant independent of $(v, \eta, \varepsilon)$.

We assume that there exist $T^{*} \in(0, T]$ and a positive constant $d_{1}$ such that $1<d_{0} \leq d_{1}$, and

$$
\sup _{0 \leq t \leq T^{*}}\left(\varepsilon\|\nabla g(t, \cdot)\|_{D^{1} \cap D^{2}}^{2}+\|V(t, \cdot)\|_{3}^{2}\right)+\int_{0}^{T^{*}} \varepsilon \sum_{i=1}^{4}\left|g \nabla^{i} v(t, \cdot)\right|_{2}^{2} \mathrm{~d} t \leq d_{1}^{2},
$$

where $T^{*}$ and $d_{1}$ will be determined later (see (4.30)), which depend only on $d_{0}$ and the fixed constants $(A, \alpha, \beta, \gamma, \delta, T)$.

Next, a series of uniform local-in-time estimates independent of $(\nu, \eta, \varepsilon)$ will be listed in Lemmas 4.2-4.3. Hereinafter, we use $C \geq 1$ to denote a generic constant depending only on the fixed constants $(A, \alpha, \beta, \gamma, \delta, T)$.

4.2.1. Uniform energy estimates on $\psi$. In order to deal with the singular elliptic operator $h^{2} L u$, we first need to make some proper estimates of $\psi$.

Lemma 4.2. Let $(h, c, u)$ be the unique strong solution of problem $(4.4)$ in $[0, T] \times \mathbb{R}^{3}$. Then

$$
\varepsilon\|\psi(t)\|_{D^{1} \cap D^{2}}^{2} \leq C d_{0}^{2} \quad \text { for } 0 \leq t \leq T_{1}=\min \left(T^{*},\left(1+d_{1}^{2}\right)^{-1}\right) .
$$

Proof. Since $\psi=\nabla h$, and equation (4.4) 1 holds, $\psi$ satisfies the following equations:

$$
\psi_{t}+\sum_{l=1}^{3} B_{l}(v) \partial_{l} \psi+B^{*}(v) \psi+\frac{\delta-1}{2}(g \nabla \operatorname{div} v+\nabla g \operatorname{div} v g)=0
$$

where $B^{*}(v)=(\nabla v)^{\top}$.

Next, let $\zeta=\left(\zeta_{1}, \zeta_{2}, \zeta_{3}\right)$ with $1 \leq|\zeta| \leq 2$ and $\zeta_{i}=0,1,2$. Applying operator $\partial_{x}^{\zeta}$ to (4.19), then multiplying by $2 \partial_{x}^{\zeta} \psi$, and integrating over $\mathbb{R}^{3}$ yield

$$
\frac{\mathrm{d}}{\mathrm{d} t}\left|\partial_{x}^{\zeta} \psi\right|_{2}^{2} \leq\left(\sum_{l=1}^{3}\left|\partial_{l} B_{l}\right|_{\infty}+\left|B^{*}\right|_{\infty}\right)\left|\partial_{x}^{\zeta} \psi\right|_{2}^{2}+\sum_{l=1}^{3}\left|\Theta_{l}\right|_{2}\left|\partial_{x}^{\zeta} \psi\right|_{2},
$$

where

$$
\begin{gathered}
\Theta_{1}:=-\partial_{x}^{\zeta}\left(B^{*} \psi\right)+B^{*} \partial_{x}^{\zeta} \psi, \quad \Theta_{2}:=\sum_{l=1}^{3}\left(-\partial_{x}^{\zeta}\left(B_{l} \partial_{l} \psi\right)+B_{l} \partial_{l} \partial_{x}^{\zeta} \psi\right), \\
\Theta_{3}:=\frac{\delta-1}{2} \partial_{x}^{\zeta} g(g \nabla \operatorname{div} v+\nabla g \operatorname{div} v) .
\end{gathered}
$$


It is direct to see

$$
\frac{\mathrm{d}}{\mathrm{d} t}\|\psi\|_{D^{1} \cap D^{2}} \leq C\|v\|_{3}\|\psi\|_{D^{1} \cap D^{2}}+C\left(\|\nabla g\|_{D^{1} \cap D^{2}}\|v\|_{3}+\left|g \nabla^{3} v\right|_{2}+\left|g \nabla^{4} v\right|_{2}\right),
$$

which, along with Gronwall's inequality and (4.17), implies

$$
\begin{aligned}
\|\psi(t)\|_{D^{1} \cap D^{2}} & \leq C\left(\left\|\psi_{0}\right\|_{D^{1} \cap D^{2}}+\int_{0}^{t}\left(d_{1}^{2} \varepsilon^{-\frac{1}{2}}+\left|g \nabla^{3} v\right|_{2}+\left|g \nabla^{4} v\right|_{2}\right) \mathrm{d} s\right) \exp \left(C d_{1} t\right) \\
& \leq C \varepsilon^{-\frac{1}{2}}\left(d_{0}+1\right) \quad \text { for } 0 \leq t \leq T_{1}=\min \left(T^{*},\left(1+d_{1}^{2}\right)^{-1}\right) .
\end{aligned}
$$

4.2.2. Uniform energy estimates on $\boldsymbol{u}$. Based on the estimates for $\psi=\nabla h$ obtained in Lemma 4.2, we now give the corresponding uniform estimates for $u$. Denote $a_{2}=\frac{2 a_{1} \delta}{\delta-1}$.

Lemma 4.3. Let $(h, c, u)$ be the unique strong solution to problem $(4.4)$ in $[0, T] \times \mathbb{R}^{3}$.

Then

$$
\|U(t)\|_{3}^{2}+\int_{0}^{t} \varepsilon \sum_{i=1}^{4}\left(\left|h \nabla^{i} u\right|_{2}^{2}+v^{2}\left|\nabla^{i} u\right|_{2}^{2}\right) \mathrm{d} s \leq C d_{0}^{2} \quad \text { for } 0 \leq t \leq T_{1} .
$$

Proof. We divide the proof into four steps.

1. Applying $\partial_{x}^{\zeta}$ to $(4.4)_{2}$, then multiplying by $2 \partial_{x}^{\zeta} U$ and integrating over $\mathbb{R}^{3}$ yield

$$
\begin{aligned}
\frac{\mathrm{d}}{\mathrm{d} t} \int & \partial_{x}^{\zeta} U \cdot\left(A_{0} \partial_{x}^{\zeta} U\right) \mathrm{d} x+2 \varepsilon a_{1} \int\left(h^{2}+v^{2}\right)\left(\alpha\left|\nabla \partial_{x}^{\zeta} u\right|^{2}+(\alpha+\beta)\left|\operatorname{div} \partial_{x}^{\zeta} u\right|^{2}\right) \mathrm{d} x \\
= & \int \operatorname{div} A\left|\partial_{x}^{\zeta} U\right|^{2} \mathrm{~d} x-2 \int \sum_{l=1}^{3}\left(\partial_{x}^{\zeta}\left(A_{l} \partial_{l} U\right)-A_{l} \partial_{x}^{\zeta} \partial_{l} U\right) \cdot \partial_{x}^{\zeta} U \mathrm{~d} x \\
& -2 \varepsilon a_{1} \alpha \int\left(\left(\nabla h^{2} \cdot \nabla \partial_{x}^{\zeta} u\right) \cdot \partial_{x}^{\zeta} u-\left(\partial_{x}^{\zeta}\left(h^{2} \Delta u\right)-h^{2} \partial_{x}^{\zeta} \Delta u\right) \cdot \partial_{x}^{\zeta} u\right) \mathrm{d} x \\
& -2 \varepsilon a_{1}(\alpha+\beta) \int\left(\nabla h^{2} \operatorname{div} \partial_{x}^{\zeta} u\right) \cdot \partial_{x}^{\zeta} u \mathrm{~d} x \\
& +2 \varepsilon a_{1}(\alpha+\beta) \int\left(\partial_{x}^{\zeta}\left(h^{2} \nabla \operatorname{div} u\right)-h^{2} \partial_{x}^{\zeta} \nabla \operatorname{div} u\right) \cdot \partial_{x}^{\zeta} u \mathrm{~d} x \\
& +\varepsilon a_{2} \int\left(\nabla h^{2} \partial_{x}^{\zeta} Q+\partial_{x}^{\zeta}\left(\nabla h^{2} Q\right)-\nabla h^{2} \partial_{x}^{\zeta} Q\right) \cdot \partial_{x}^{\zeta} u \mathrm{~d} x \\
= & : \sum_{i=1}^{8} I_{i} .
\end{aligned}
$$

2. We need to consider the terms on the right-hand side of (4.22) when $|\zeta| \leq 3$. It follows from the Gagliardo-Nirenberg inequality and Hölder's inequality that

$$
I_{1}=\int \operatorname{div} A\left|\partial_{x}^{\zeta} U\right|^{2} \mathrm{~d} x \leq C|\operatorname{div} A|_{\infty}\left|\partial_{x}^{\zeta} U\right|_{2}^{2} \leq C d_{1}\left|\partial_{x}^{\zeta} U\right|_{2}^{2} \quad \text { for }|\zeta| \leq 3 .
$$


Similarly, for $I_{2}$, using Lemma A.5, we have

$$
\begin{aligned}
I_{2} & =-2 \sum_{l=1}^{3} \int\left(\partial_{x}^{\zeta}\left(A_{l} \partial_{l} U\right)-A_{l} \partial_{x}^{\zeta} \partial_{l} U\right) \cdot \partial_{x}^{\zeta} U \mathrm{~d} x \\
& \leq C|\nabla V|_{\infty}|\nabla U|_{2}^{2} \leq C d_{1}|\nabla U|_{2}^{2} \text { for }|\zeta|=1 \\
I_{2} & \leq C\left(|\nabla V|_{\infty}\left|\nabla^{2} U\right|_{2}+\left|\nabla^{2} V\right|_{3}|\nabla U|_{6}\right)\left|\nabla^{2} U\right|_{2} \\
& \leq C d_{1}\|\nabla U\|_{1}^{2} \text { for }|\zeta|=2 \\
I_{2} & \leq C\left(|\nabla V|_{\infty}\left|\nabla^{3} U\right|_{2}+\left|\nabla^{3} V\right|_{2}|\nabla U|_{\infty}\right)\left|\nabla^{3} U\right|_{2} \\
& \leq C d_{1}\|\nabla U\|_{2}^{2} \text { for }|\zeta|=3 .
\end{aligned}
$$

For $I_{3}$ and $I_{5}$, it follows from Lemma 4.2, the Gagliardo-Nirenberg inequality, the Hölder inequality, and the Young inequality that

$$
\begin{aligned}
I_{3}+I_{5} & =-2 \varepsilon a_{1} \int\left(\alpha \nabla h^{2} \nabla \partial_{x}^{\zeta} u+(\alpha+\beta) \nabla h^{2} \operatorname{div} \partial_{x}^{\zeta} u\right) \cdot \partial_{x}^{\zeta} u \mathrm{~d} x \\
& \leq C \varepsilon a_{1}|\psi|_{\infty}\left|h \nabla \partial_{x}^{\zeta} u\right|_{2}\left|\partial_{x}^{\zeta} u\right|_{2} \\
& \leq \frac{\varepsilon a_{1} \alpha}{16}\left|h \nabla \partial_{x}^{\zeta} u\right|_{2}^{2}+C d_{0}^{2}\left|\partial_{x}^{\zeta} u\right|_{2}^{2} \quad \text { for }|\zeta| \leq 3
\end{aligned}
$$

Similarly, for $I_{4}$ and $I_{7}$, it follows from Lemma 4.2 that

$$
\begin{aligned}
I_{4}= & 2 \varepsilon a_{1} \alpha \int\left(\partial_{x}^{\zeta}\left(h^{2} \triangle u\right)-h^{2} \partial_{x}^{\zeta} \triangle u\right) \cdot \partial_{x}^{\zeta} u \mathrm{~d} x \\
\leq & C \varepsilon a_{1} \alpha|\psi|_{\infty}|h \triangle u|_{2}|\nabla u|_{2} \\
\leq & \frac{\varepsilon a_{1} \alpha}{16}\left|h \nabla^{2} u\right|_{2}^{2}+C d_{0}^{2}|\nabla u|_{2}^{2} \quad \text { for }|\zeta|=1, \\
I_{4} \leq & C \varepsilon a_{1} \alpha\left(|\psi|_{\infty}\left|h \nabla^{3} u\right|_{2}+|\psi|_{\infty}^{2}|\Delta u|_{2}+|\nabla \psi|_{3}\left|h \nabla^{2} u\right|_{6}\right)\left|\nabla^{2} u\right|_{2} \\
\leq & \frac{\varepsilon a_{1} \alpha}{16}\left|h \nabla^{3} u\right|_{2}^{2}+C d_{0}^{2}\left\|\nabla^{2} u\right\|_{1}^{2} \quad \text { for }|\zeta|=2, \\
I_{4} \leq & C \varepsilon a_{1} \alpha\left(\left|\nabla^{2} \psi\right|_{2}\left|h \nabla^{2} u\right|_{\infty}+|\nabla \psi|_{3}\left|h \nabla^{3} u\right|_{6}+|\psi|_{\infty}|\nabla \psi|_{3}\left|\nabla^{2} u\right|_{6}\right)\left|\nabla^{3} u\right|_{2} \\
& +C \varepsilon a_{1} \alpha\left(|\psi|_{\infty}^{2}\left|\nabla^{3} u\right|_{2}+|\psi|_{\infty}\left|h \nabla^{4} u\right|_{2}\right)\left|\nabla^{3} u\right|_{2} \\
\leq & \frac{\varepsilon a_{1} \alpha}{16}\left(\left|h \nabla^{3} u\right|_{2}^{2}+\left|h \nabla^{4} u\right|_{2}^{2}\right)+C d_{0}^{2}\left\|\nabla^{2} u\right\|_{1}^{2} \quad \text { for }|\zeta|=3, \\
I_{7}= & \varepsilon a_{2} \int\left(\nabla h^{2} \partial_{x}^{\zeta} Q(u)\right) \cdot \partial_{x}^{\zeta} u \mathrm{~d} x \leq C \varepsilon a_{1}|\psi|_{\infty}\left|h \nabla^{|\zeta|+1} u\right|_{2}\left|\partial_{x}^{\zeta} u\right|_{2} \\
\leq & \frac{\varepsilon a_{1} \alpha}{16}\left|h \nabla^{|\zeta|+1} u\right|_{2}^{2}+C d_{0}^{2}\left|\partial_{x}^{\zeta} u\right|_{2}^{2} \quad \text { for }|\zeta| \leq 3 .
\end{aligned}
$$

Because of the same structure, the above estimates of $I_{4}$ also hold for the term

$$
I_{6}=2 \varepsilon a_{1}(\alpha+\beta) \int\left(\partial_{x}^{\zeta}\left(h^{2} \nabla \operatorname{div} u\right)-h^{2} \partial_{x}^{\zeta} \nabla \operatorname{div} u\right) \cdot \partial_{x}^{\zeta} u \mathrm{~d} x .
$$


3. For $I_{8}$, it follows from Lemma 4.2 that

$$
\begin{aligned}
I_{8}= & \varepsilon a_{2} \int\left(\partial_{x}^{\zeta}\left(\nabla h^{2} Q(u)\right)-\nabla h^{2} \partial_{x}^{\zeta} Q(u)\right) \cdot \partial_{x}^{\zeta} u \mathrm{~d} x \\
\leq & C \varepsilon a_{2}\left(|\psi|_{\infty}^{2}|\nabla u|_{2}^{2}+|\nabla \psi|_{6}|h \nabla u|_{2}|\nabla u|_{3}\right) \\
\leq & \frac{\varepsilon a_{1} \alpha}{16}|h \nabla u|_{2}^{2}+C d_{0}^{2}\|\nabla u\|_{1}^{2} \quad \text { for }|\zeta|=1, \\
I_{8} \leq & C \varepsilon a_{2}\left(\left|\nabla^{2} \psi\right|_{2}|h \nabla u|_{\infty}+|\psi|_{\infty}|\nabla \psi|_{2}|\nabla u|_{\infty}\right)\left|\nabla^{2} u\right|_{2} \\
& +C \varepsilon a_{2}\left(|\nabla \psi|_{3}\left|h \nabla^{2} u\right|_{6}+|\psi|_{\infty}^{2}\left|\nabla^{2} u\right|_{2}\right)\left|\nabla^{2} u\right|_{2} \\
\leq & \frac{\varepsilon a_{1} \alpha}{16}\left(\left|h \nabla^{2} u\right|_{2}^{2}+\left|h \nabla^{3} u\right|_{2}^{2}\right)+C d_{0}^{2}\|\nabla u\|_{2}^{2} \text { for }|\zeta|=2 .
\end{aligned}
$$

On the other hand, when $|\zeta|=3$,

$$
\begin{aligned}
& \partial_{x}^{\zeta}\left(\nabla h^{2} Q(u)\right)-\nabla h^{2} \partial_{x}^{\zeta} Q(u) \\
& \quad=\sum_{i=1}^{3} C_{i 1} \partial_{x}^{\zeta^{i}} \nabla h^{2} \partial_{x}^{\zeta-\zeta^{i}} Q(u)+\sum_{i=1}^{3} C_{i 2} \partial_{x}^{\zeta-\zeta^{i}} \nabla h^{2} \partial_{x}^{\zeta^{i}} Q(u)+\partial_{x}^{\zeta} \nabla h^{2} Q(u),
\end{aligned}
$$

where $C_{i j}, i=1,2,3, j=1,2$, are all constants, and $\zeta=\zeta^{1}+\zeta^{2}+\zeta^{3}$ with $\zeta^{i} \in \mathbb{R}^{3}$ as a multi-index satisfying $\left|\zeta^{i}\right|=1, i=1,2,3$. Then $I_{8}:=I_{81}+I_{82}+I_{83}$ can be estimated as follows:

$$
\begin{aligned}
I_{81}= & \varepsilon a_{2} \int \sum_{i=1}^{3} C_{i 1}\left(\partial_{x}^{\zeta^{i}} \nabla h^{2} \partial_{x}^{\zeta-\zeta^{i}} Q(u)\right) \cdot \partial_{x}^{\zeta} u \mathrm{~d} x \\
\leq & C \varepsilon a_{1}\left(|\psi|_{\infty}^{2}\left|\nabla^{3} u\right|_{2}+|\nabla \psi|_{3}\left|h \nabla^{3} u\right|_{6}\right)\left|\nabla^{3} u\right|_{2} \\
\leq & \frac{\varepsilon a_{1} \alpha}{16}\left|h \nabla^{4} u\right|_{2}^{2}+C d_{0}^{2}\left|\nabla^{3} u\right|_{2}^{2}, \\
I_{82}= & \varepsilon a_{2} \int \sum_{i=1}^{3} C_{i 2}\left(\partial_{x}^{\zeta-\zeta^{i}} \nabla h^{2} \partial_{x}^{\zeta^{i}} Q(u)\right) \cdot \partial_{x}^{\zeta} u \mathrm{~d} x \\
\leq & C \varepsilon a_{1}\left(\left|h \nabla^{2} u\right|_{\infty}\left|\nabla^{2} \psi\right|_{2}+|\psi|_{\infty}|\nabla \psi|_{6}\left|\nabla^{2} u\right|_{3}\right)\left|\nabla^{3} u\right|_{2} \\
\leq & \frac{\varepsilon a_{1} \alpha}{16}\left(\left|h \nabla^{3} u\right|_{2}^{2}+\left|h \nabla^{4} u\right|_{2}^{2}\right)+C d_{0}^{2}\left\|\nabla^{2} u\right\|_{1}^{2}, \\
I_{83}, & 2 \varepsilon a_{1} \int\left(\partial_{x}^{\zeta} \nabla h^{2} Q(u)\right) \cdot \partial_{x}^{\zeta} u \mathrm{~d} x \\
\leq & C \varepsilon a_{1}\left(\left|h \nabla^{2} u\right|_{\infty}\left|\nabla^{3} u\right|_{2}+\left|h \nabla^{4} u\right|_{2}|\nabla u|_{\infty}\right)\left|\nabla^{2} \psi\right|_{2} \\
& +C \varepsilon a_{1}\left(|\psi|_{\infty}\left|\nabla^{2} \psi\right|_{2}+|\nabla \psi|_{3}|\nabla \psi|_{6}\right)|\nabla u|_{\infty}\left|\nabla^{3} u\right|_{2} \\
\leq & \frac{\varepsilon a_{1} \alpha}{16}\left(\left|h \nabla^{3} u\right|_{2}^{2}+\left|h \nabla^{4} u\right|_{2}^{2}\right)+C d_{0}^{2}\left\|\nabla^{2} u\right\|_{1}^{2},
\end{aligned}
$$

where we have performed integration by parts for $I_{83}$. 
4. From (4.22)-(4.28), along with the Gronwall inequality, we have

$$
\begin{aligned}
\|U(t)\|_{3}^{2}+\varepsilon \int_{0}^{t} \sum_{i=1}^{4}\left(\left|h \nabla^{i} u\right|_{2}^{2}+v^{2}\left|\nabla^{i} u\right|_{2}^{2}\right) \mathrm{d} s & \leq\left\|U_{0}\right\|_{3}^{2} \exp \left(C d_{1}^{2} t\right) \\
& \leq C d_{0}^{2} \quad \text { for } 0 \leq t \leq T_{1} .
\end{aligned}
$$

This completes the proof.

Then, from Lemmas 4.2-4.3, for

$$
0 \leq t \leq T_{1}=\min \left(T^{*},\left(1+d_{1}\right)^{-2}\right),
$$

we have

$$
\varepsilon\|\psi(t)\|_{D^{1} \cap D^{2}}^{2}+\|U(t)\|_{3}^{2}+\varepsilon \sum_{i=1}^{4} \int_{0}^{t}\left(\left|h \nabla^{i} u\right|_{2}^{2}+v^{2}\left|\nabla^{i} u\right|_{2}^{2}\right) \mathrm{d} s \leq C d_{0}^{2} .
$$

Therefore, defining

$$
T^{*}=\min \left(T,\left(1+d_{1}^{2}\right)^{-1}\right), \quad d_{1}=C^{\frac{1}{2}} d_{0}
$$

we have

$$
\begin{gathered}
\varepsilon\|\psi(t)\|_{D^{1} \cap D^{2}}^{2}+\|U(t)\|_{3}^{2}+\varepsilon \sum_{i=1}^{4} \int_{0}^{t}\left(\left|h \nabla^{i} u\right|_{2}^{2}+v^{2}\left|\nabla^{i} u\right|_{2}^{2}\right) \mathrm{d} s \\
\leq d_{1}^{2} \quad \text { for } 0 \leq t \leq T^{*} .
\end{gathered}
$$

In other words, given fixed $d_{0}$ and $T$, there are positive constants $T^{*}$ and $d_{1}$, depending only on $d_{0}$ and $T$, such that, if (4.17) holds for $(g, v)$, then (4.31) holds for the strong solution $(h, \psi, c, u)$ of problem (4.4) in $\left[0, T^{*}\right] \times \mathbb{R}^{3}$.

\subsection{Construction of the nonlinear approximation solutions}

In this subsection, based on the assumption that $v>0$ and $h_{0} \leq \eta^{\imath}$ for some $\eta>0$, we now give the local-in-time well-posedness of the following nonlinear Cauchy problem:

$$
\left\{\begin{array}{l}
h_{t}+u \cdot \nabla h+\frac{\delta-1}{2} h \operatorname{div} u=0, \\
A_{0} U_{t}+\sum_{j=1}^{3} A_{j}(U) \partial_{j} U=-\varepsilon F(v, h, u)+\varepsilon G(h, \psi, u), \\
\left.(h, c, u)\right|_{t=0}=\left((A \gamma)^{-\frac{\imath}{2}}\left(c_{0}+\eta\right)^{\iota}, c_{0}, u_{0}\right)(x) \text { for } x \in \mathbb{R}^{3},
\end{array}\right.
$$

where $\psi=\nabla h$ satisfies

$$
\psi_{t}+\sum_{l=1}^{3} B_{l}(u) \partial_{l} \psi+B(u) \psi+\frac{\delta-1}{2} h \nabla \operatorname{div} u=0 .
$$


Theorem 4.2. Let (2.1) hold and $(v, \eta, \varepsilon) \in(0,1] \times(0,1] \times(0,1]$. If the initial data $\left(c_{0}, h_{0}, u_{0}\right)$ satisfy (4.13), then there exist $T_{*}>0$ and a unique classical solution $(h, c, u)$ of problem (4.32) in $\left[0, T_{*}\right] \times \mathbb{R}^{3}$ such that (4.14) and the uniform a priori estimates $(4.31)$ with $T^{*}$ replaced by $T_{*}$ hold, where $T_{*}$ is independent of $(v, \eta, \varepsilon)$.

The proof is based on an iteration scheme and the conclusions obtained in Sections 4.1-4.2. As in Section 4.2, we define the same constants $d_{i}, i=0,1$.

Let $\left(h^{0}, c^{0}, u^{0}\right)$ be the solution of the Cauchy problem in $(0, \infty) \times \mathbb{R}^{3}$ :

$$
\left\{\begin{array}{l}
X_{t}+u_{0} \cdot \nabla X=0 \\
Y_{t}+u_{0} \cdot \nabla Y=0 \\
Z_{t}-\varepsilon\left(X^{2}+v^{2}\right) \Delta Z=0 \\
\left.(X, Y, Z)\right|_{t=0}=\left((A \gamma)^{-\frac{\iota}{2}}\left(c_{0}+\eta\right)^{\iota}, c_{0}, u_{0}\right)(x) \quad \text { for } x \in \mathbb{R}^{3} .
\end{array}\right.
$$

Choose $T^{* *} \in\left(0, T^{*}\right]$ small enough such that, for $0 \leq t \leq T^{* *}$,

$$
\begin{aligned}
& \varepsilon\left\|\nabla h^{0}(t)\right\|_{D^{1} \cap D^{2}}^{2}+\left\|\left(c^{0}, u^{0}\right)(t)\right\|_{3}^{2} \\
& \quad+\varepsilon \sum_{i=1}^{4} \int_{0}^{t}\left(\left|h^{0} \nabla^{i} u^{0}\right|_{2}^{2}+v^{2}\left|\nabla^{i} u^{0}\right|_{2}^{2}\right) \mathrm{d} s \leq d_{1}^{2} .
\end{aligned}
$$

Proof of Theorem 4.2. The desired existence, uniqueness, and time continuity can be proved in the following three steps.

1. Existence. Let the first step of our iteration be $(g, \varphi, v)=\left(h^{0}, c^{0}, u^{0}\right)$. Then we can obtain a classical solution $\left(h^{1}, c^{1}, u^{1}\right)$ of problem (4.4) so that $\psi^{1}:=\nabla h^{1}$ is defined. Inductively, we construct approximate sequences $\left(h^{k+1}, c^{k+1}, u^{k+1}\right)$ as follows: given $\left(h^{k}, c^{k}, u^{k}\right)$ for $k \geq 1$, define $\left(h^{k+1}, c^{k+1}, u^{k+1}\right)$ by solving the problem

$$
\left\{\begin{array}{l}
h_{t}^{k+1}+u^{k} \cdot \nabla h^{k+1}+\frac{\delta-1}{2} h^{k} \operatorname{div} u^{k}=0, \\
A_{0} U_{t}^{k+1}+\sum_{l=1}^{3} A_{l}\left(U^{k}\right) \partial_{l} U^{k+1} \\
\quad=-\varepsilon F\left(v, h^{k+1}, u^{k+1}\right)+\varepsilon G\left(h^{k+1}, \psi^{k+1}, u^{k+1}\right), \\
\left.\left(h^{k+1}, c^{k+1}, u^{k+1}\right)\right|_{t=0}=\left((A \gamma)^{-\frac{l}{2}}\left(c_{0}+\eta\right)^{\iota}, c_{0}, u_{0}\right)(x) \quad \text { for } x \in \mathbb{R}^{3},
\end{array}\right.
$$

where $\psi^{k+1}=\nabla h^{k+1}$ satisfy the following equations (cf. (4.19)):

$$
\psi_{t}^{k+1}+\sum_{l=1}^{3} B_{l}\left(u^{k}\right) \partial_{l} \psi^{k+1}+B^{*}\left(u^{k}\right) \psi^{k+1}+\frac{\delta-1}{2} \nabla\left(h^{k} \operatorname{div} u^{k}\right)=0 .
$$

Then the solution sequences $\left(h^{k}, c^{k}, u^{k}\right), k=1,2, \ldots$, satisfy the uniform estimates (4.31). 
Next we prove that the whole sequence $\left(h^{k}, \psi^{k}, c^{k}, u^{k}\right)$ converges strongly to a limit $(h, \psi, c, u)=(h, \nabla h, c, u)$ in some strong sense. Denote

$$
\begin{gathered}
\bar{h}^{k+1}:=h^{k+1}-h^{k}, \quad \bar{\psi}^{k+1}:=\psi^{k+1}-\psi^{k}=\nabla \bar{h}^{k+1}, \\
\bar{c}^{k+1}:=c^{k+1}-c^{k}, \quad \bar{u}^{k+1}:=u^{k+1}-u^{k}, \quad \bar{U}^{k+1}=\left(\bar{c}^{k+1}, \bar{u}^{k+1}\right) .
\end{gathered}
$$

Then, from (4.36), we have

$$
\left\{\begin{aligned}
\bar{h}_{t}^{k+1}+ & u^{k} \cdot \nabla \bar{h}^{k+1}+\bar{u}^{k} \cdot \psi^{k}+\frac{\delta-1}{2}\left(\bar{h}^{k} \operatorname{div} u^{k-1}+h^{k} \operatorname{div} \bar{u}^{k}\right)=0 \\
\bar{\psi}_{t}^{k+1}+ & \sum_{l=1}^{3} B_{l}\left(u^{k}\right) \partial_{l} \bar{\psi}^{k+1}+B^{*}\left(u^{k}\right) \bar{\psi}^{k+1} \\
= & -\frac{\delta-1}{2}\left(\bar{h}^{k} \nabla \operatorname{div} u^{k-1}+h^{k} \nabla \operatorname{div} \bar{u}^{k}\right) \\
& -\sum_{l=1}^{3} B_{l}\left(\bar{u}^{k}\right) \partial_{l} \psi^{k}-B^{*}\left(\bar{u}^{k}\right) \psi^{k}-\frac{\delta-1}{2}\left(\bar{\psi}^{k} \operatorname{div} u^{k-1}+\psi^{k} \operatorname{div} \bar{u}^{k}\right) \\
A_{0} \bar{U}_{t}^{k+1} & +\sum_{l=1}^{3} A_{l}\left(U^{k}\right) \partial_{l} \bar{U}^{k+1}+\varepsilon F\left(v, h^{k+1}, \bar{u}^{k+1}\right) \\
= & -\sum_{l=1}^{3} A_{l}\left(\bar{U}^{k}\right) \partial_{l} U^{k}-\varepsilon\left(F\left(v, h^{k+1}, u^{k}\right)-F\left(v, h^{k}, u^{k}\right)\right) \\
& +\varepsilon\left(G\left(h^{k+1}, \bar{\psi}^{k+1}, u^{k+1}\right)+G\left(h^{k+1}, \psi^{k}, \bar{u}^{k+1}\right)+G\left(\bar{h}^{k+1}, \psi^{k}, u^{k}\right)\right) .
\end{aligned}\right.
$$

First, for $\bar{h}^{k+1}$, we state the following lemma:

Lemma 4.4. We have

$$
\bar{h}^{k+1} \in L^{\infty}\left(\left[0, T^{* *}\right] ; H^{3}\left(\mathbb{R}^{3}\right)\right) \text { for } k=1,2, \ldots
$$

Remark 4.1. This lemma is important for our limit process from the linear problem to the nonlinear one, and helps us to deal with the cancellation of the most singular terms in the limit process. Its proof can be found in Remark 4.2 at the end of this subsection.

Based on Lemma 4.4, multiplying (4.38) 1 by $2 \bar{h}^{k+1}$ and then integrating over $\mathbb{R}^{3}$, we have

$$
\begin{aligned}
\varepsilon \frac{\mathrm{d}}{\mathrm{d} t}\left|\bar{h}^{k+1}\right|_{2}^{2} \leq & C \varepsilon\left(\left|\nabla u^{k}\right|_{\infty}\left|\bar{h}^{k+1}\right|_{2}+\left|\psi^{k}\right|_{6}\left|\bar{u}^{k}\right|_{3}\right)\left|\bar{h}^{k+1}\right|_{2} \\
& +C \varepsilon\left(\left|\bar{h}^{k}\right|_{2}\left|\nabla u^{k-1}\right|_{\infty}+\left|h^{k} \nabla \bar{u}^{k}\right|_{2}\right)\left|\bar{h}^{k+1}\right|_{2} \\
\leq & C \sigma^{-1} \varepsilon\left|\bar{h}^{k+1}\right|_{2}^{2}+\sigma\left(\left\|\bar{u}^{k}\right\|_{1}^{2}+\varepsilon\left|\bar{h}^{k}\right|_{2}^{2}+\varepsilon\left|h^{k} \nabla \bar{u}^{k}\right|_{2}^{2}\right)
\end{aligned}
$$

where $\sigma \in\left(0, \frac{1}{10}\right)$ is a constant to be determined later. 
Then multiplying $(4.38)_{2}$ by $2 \bar{\psi}^{k+1}$ and integrating over $\mathbb{R}^{3}$ yield

$$
\begin{aligned}
\varepsilon \frac{\mathrm{d}}{\mathrm{d} t}\left|\bar{\psi}^{k+1}\right|_{2}^{2} \leq & C \varepsilon\left|\nabla u^{k}\right|_{\infty}\left|\bar{\psi}^{k+1}\right|_{2}^{2}+C \varepsilon\left(\left|\nabla^{2} u^{k-1}\right|_{3}\left|\bar{h}^{k}\right|_{6}+\left|h^{k} \nabla^{2} \bar{u}^{k}\right|_{2}\right)\left|\bar{\psi}^{k+1}\right|_{2} \\
& +C \varepsilon\left(\left|\bar{u}^{k}\right|_{6}\left|\nabla \psi^{k}\right|_{3}+\left|\nabla \bar{u}^{k}\right|_{2}\left|\psi^{k}\right|_{\infty}+\left|\bar{\psi}^{k}\right|_{2}\left|\nabla u^{k-1}\right|_{\infty}\right)\left|\bar{\psi}^{k+1}\right|_{2} \\
\leq & C \sigma^{-1} \varepsilon\left|\bar{\psi}^{k+1}\right|_{2}^{2}+\sigma\left(\left\|\bar{u}^{k}\right\|_{1}^{2}+\varepsilon\left|\bar{\psi}^{k}\right|_{2}^{2}+\varepsilon\left|h^{k} \nabla^{2} \bar{u}^{k}\right|_{2}^{2}\right) .
\end{aligned}
$$

Next, multiplying (4.38) 3 by $2 \bar{U}^{k+1}$ and integrating over $\mathbb{R}^{3}$, we have

$$
\begin{aligned}
\frac{\mathrm{d}}{\mathrm{d} t} \int & \bar{U}^{k+1} \cdot\left(A_{0} \bar{U}^{k+1}\right) \mathrm{d} x+2 \varepsilon a_{1} \int \alpha\left(\left|h^{k+1} \nabla \bar{u}^{k+1}\right|^{2}+v^{2}\left|\nabla \bar{u}^{k+1}\right|^{2}\right) \mathrm{d} x \\
& +2 \varepsilon a_{1} \int(\alpha+\beta)\left(\left|h^{k+1} \operatorname{div} \bar{u}^{k+1}\right|^{2}+v^{2}\left|\operatorname{div} \bar{u}^{k+1}\right|^{2}\right) \mathrm{d} x \\
= & \int \operatorname{div} A\left(U^{k}\right)\left|\bar{U}^{k+1}\right|_{2}^{2} \mathrm{~d} x-2 \sum_{l=1}^{3} \int\left(A_{l}\left(\bar{U}^{k}\right) \partial_{l} U^{k}\right) \cdot \bar{U}^{k+1} \mathrm{~d} x \\
& -2 \varepsilon a_{1} \int\left(\alpha\left(\nabla\left(h^{k+1}\right)^{2} \cdot \nabla \bar{u}^{k+1}\right)+(\alpha+\beta)\left(\nabla\left(h^{k+1}\right)^{2} \operatorname{div} \bar{u}^{k+1}\right)\right) \cdot \bar{u}^{k+1} \mathrm{~d} x \\
& +2 \varepsilon a_{1} \int\left(-\bar{h}^{k+1}\left(h^{k+1}+h^{k}\right) L u^{k}+\frac{2 \delta}{\delta-1} h^{k+1} \bar{\psi}^{k+1} Q\left(u^{k+1}\right)\right) \cdot \bar{u}^{k+1} \mathrm{~d} x \\
& +\frac{4 \varepsilon a_{1} \delta}{\delta-1} \int\left(h^{k+1} \psi^{k} Q\left(\bar{u}^{k+1}\right)+\bar{h}^{k+1} \psi^{k} Q\left(u^{k}\right)\right) \cdot \bar{u}^{k+1} \mathrm{~d} x \\
\leq & C\left|\nabla U^{k}\right|_{\infty}\left|\bar{U}^{k+1}\right|_{2}\left(\left|\bar{U}^{k+1}\right|_{2}+\left|\bar{U}^{k}\right|_{2}\right)+C \varepsilon\left|\psi^{k+1}\right|_{\infty}\left|h^{k+1} \nabla \bar{u}^{k+1}\right|_{2}\left|\bar{u}^{k+1}\right|_{2} \\
& +C \varepsilon\left(\left|\bar{h}^{k+1}\right|_{6}^{2}\left|L u^{k}\right|_{2}+\left|\bar{h}^{k+1}\right|_{2}\left|h^{k} L u^{k}\right|_{6}+\left|\bar{\psi}^{k+1}\right|_{2}\left|h^{k+1} \nabla u^{k+1}\right|_{6}\right)\left|\bar{u}^{k+1}\right|_{3} \\
& +C \varepsilon\left(\left|h^{k+1} \nabla \bar{u}^{k+1}\right|_{2}\left|\psi^{k}\right|_{\infty}+\left|\psi^{k}\right|_{\infty}\left|\nabla u^{k}\right|_{\infty}\left|\bar{h}^{k+1}\right|_{2}\right)\left|\bar{u}^{k+1}\right|_{2},
\end{aligned}
$$

where we have used the following fact:

$$
\begin{aligned}
\int \bar{h}^{k+1} h^{k+1} L u^{k} \cdot \bar{u}^{k+1} \mathrm{~d} x & =\int \bar{h}^{k+1}\left(\bar{h}^{k+1}+h^{k}\right) L u^{k} \cdot \bar{u}^{k+1} \mathrm{~d} x \\
& \leq C\left(\left|\bar{h}^{k+1}\right|_{6}^{2}\left|L u^{k}\right|_{2}+\left|\bar{h}^{k+1}\right|_{2}\left|h^{k} L u^{k}\right|_{6}\right)\left|\bar{u}^{k+1}\right|_{3} .
\end{aligned}
$$

Now, applying operator $\partial_{x}^{\zeta}(|\zeta|=1)$ to $(4.38)_{3}$, multiplying by $2 \partial_{x}^{\zeta} \bar{U}^{k+1}$, and integrating over $\mathbb{R}^{3}$ yield

$$
\begin{aligned}
& \frac{\mathrm{d}}{\mathrm{d} t} \int \partial_{x}^{\zeta} \bar{U}^{k+1} \cdot\left(A_{0} \partial_{x}^{\zeta} \bar{U}^{k+1}\right) \mathrm{d} x+2 \varepsilon a_{1} \int \alpha\left(\left|h^{k+1} \nabla \partial_{x}^{\zeta} \bar{u}^{k+1}\right|^{2}+v^{2}\left|\nabla \partial_{x}^{\zeta} \bar{u}^{k+1}\right|^{2}\right) \mathrm{d} x \\
& +2 \varepsilon a_{1} \int(\alpha+\beta)\left(\left|h^{k+1} \operatorname{div} \partial_{x}^{\zeta} \bar{u}^{k+1}\right|^{2}+v^{2}\left|\operatorname{div} \partial_{x}^{\zeta} \bar{u}^{k+1}\right|^{2}\right) \mathrm{d} x
\end{aligned}
$$




$$
\begin{aligned}
= & \int \operatorname{div} A\left(U_{k}\right)\left|\partial_{x}^{\zeta} \bar{U}^{k+1}\right|^{2} \mathrm{~d} x-2 \sum_{l=1}^{3} \int \partial_{x}^{\zeta}\left(A_{l}\left(\bar{U}^{k}\right) \partial_{l} U^{k}\right) \cdot \partial_{x}^{\zeta} \bar{U}^{k+1} \mathrm{~d} x \\
& +2 \sum_{l=1}^{3} \int\left(A_{l}\left(U^{k}\right) \partial_{l} \partial_{x}^{\zeta} \bar{U}^{k+1}-\partial_{x}^{\zeta}\left(A_{l}\left(U^{k}\right) \partial_{l} \bar{U}^{k+1}\right)\right) \cdot \partial_{x}^{\zeta} \bar{U}^{k+1} \mathrm{~d} x \\
& +2 \varepsilon a_{1} \int \partial_{x}^{\zeta}\left(-\bar{h}^{k+1}\left(h^{k+1}+h^{k}\right) L u^{k}+G\left(h^{k+1}, \bar{\psi}^{k+1}, u^{k+1}\right)\right) \cdot \partial_{x}^{\zeta} \bar{u}^{k+1} \mathrm{~d} x \\
& +2 \varepsilon a_{1} \int \partial_{x}^{\zeta}\left(G\left(h^{k+1}, \psi^{k}, \bar{u}^{k+1}\right)+G\left(\bar{h}^{k+1}, \psi^{k}, u^{k}\right)\right) \cdot \partial_{x}^{\zeta} \bar{u}^{k+1} \mathrm{~d} x \\
:= & J^{*} .
\end{aligned}
$$

It follows from integration by parts and Hölder's inequality that

$$
\begin{aligned}
J^{*} \leq & C\left(\left|\nabla U^{k}\right|_{\infty}\left(\left|\nabla \bar{U}^{k+1}\right|_{2}+\left|\nabla \bar{U}^{k}\right|_{2}\right)+\left|\bar{U}^{k}\right|_{6}\left|\nabla^{2} U^{k}\right|_{3}\right)\left|\nabla \bar{U}^{k+1}\right|_{2} \\
& +C \varepsilon\left(\left|\bar{h}^{k+1}\right|_{6}\left|h^{k+1} \nabla^{2} \bar{u}^{k+1}\right|_{2}\left|L u^{k}\right|_{3}+\left|h^{k} L u^{k}\right|_{\infty}\left|\bar{\psi}^{k+1}\right|_{2}\left|\nabla \bar{u}^{k+1}\right|_{2}\right) \\
& +C \varepsilon\left(\left|\bar{h}^{k+1}\right|_{3}\left|h^{k} \nabla^{3} u^{k}\right|_{6}\left|\nabla \bar{u}^{k+1}\right|_{2}+\left|\bar{h}^{k+1}\right|_{6}\left|\psi^{k}\right|_{\infty}\left|\nabla^{2} u^{k}\right|_{3}\left|\nabla \bar{u}^{k+1}\right|_{2}\right) \\
& +C \varepsilon\left(\left|\bar{\psi}^{k+1}\right|_{2}\left|\nabla u^{k+1}\right|_{\infty}+\left|\psi^{k}\right|_{\infty}\left|\nabla \bar{u}^{k+1}\right|_{2}\right)\left|h^{k+1} \nabla^{2} \bar{u}^{k+1}\right|_{2} \\
& +C \varepsilon\left(\left|\nabla \psi^{k}\right|_{3}\left|\bar{h}^{k+1}\right|_{6}+\left|\psi^{k}\right|_{\infty}\left|\bar{\psi}^{k+1}\right|_{2}\right)\left|\nabla u^{k}\right|_{\infty}\left|\nabla \bar{u}^{k+1}\right|_{2} .
\end{aligned}
$$

Combining (4.41)-(4.44) with the Young inequality, we have

$$
\begin{gathered}
\frac{\mathrm{d}}{\mathrm{d} t}\left\|\bar{U}^{k+1}\right\|_{1}^{2}+\varepsilon a_{1}\left(v^{2}\left\|\nabla \bar{u}^{k+1}\right\|_{1}^{2}+\left|h^{k+1} \nabla \bar{u}^{k+1}\right|_{2}^{2}+\left|h^{k+1} \nabla^{2} \bar{u}^{k+1}\right|_{2}^{2}\right) \\
\leq \Theta_{\sigma, \varepsilon}^{k}(t)\left(\left\|\bar{U}^{k+1}\right\|_{1}^{2}+\varepsilon\left|\bar{\psi}^{k+1}\right|_{2}^{2}+\varepsilon\left|\bar{h}^{k+1}\right|_{2}^{2}\right)+\sigma\left\|\bar{U}^{k}\right\|_{1}^{2},
\end{gathered}
$$

where $\Theta_{\sigma, \varepsilon}^{k}(t)$ satisfies

$$
\int_{0}^{t} \Theta_{\sigma, \varepsilon}^{k}(s) \mathrm{d} s \leq C+C_{\sigma} t \quad \text { for } t \in\left(0, T^{* *}\right] .
$$

Finally, let

$$
\Gamma^{k+1}(t)=\sup _{0 \leq s \leq t}\left(\varepsilon\left|\bar{h}^{k+1}(s)\right|_{2}^{2}+\varepsilon\left|\bar{\psi}^{k+1}(s)\right|_{2}^{2}+\left\|\bar{U}^{k+1}(s)\right\|_{1}^{2}\right) .
$$

Combining (4.39)-(4.40) and (4.45) with the Gronwall inequality leads to

$$
\begin{aligned}
& \Gamma^{k+1}(t)+\varepsilon a_{1} \int_{0}^{t}\left(v^{2}\left\|\nabla \bar{u}^{k+1}\right\|_{1}^{2}+\left|h^{k+1} \nabla \bar{u}^{k+1}\right|_{2}^{2}+\left|h^{k+1} \nabla^{2} \bar{u}^{k+1}\right|_{2}^{2}\right) \mathrm{d} s \\
& \quad \leq C \sigma\left(\varepsilon \int_{0}^{t}\left(\left|h^{k} \nabla \bar{u}^{k}\right|_{2}^{2}+\left|h^{k} \nabla^{2} \bar{u}^{k}\right|_{2}^{2}\right) \mathrm{d} s+t \sup _{0 \leq s \leq t} \Gamma^{k}(t)\right) \exp \left(C+C_{\sigma} t\right) .
\end{aligned}
$$

Choose $\sigma>0$ and $T_{*} \in\left(0, T^{* *}\right)$ small enough such that

$$
C \sigma \exp C \leq \min \left(\frac{1}{8}, \frac{a_{1}}{8}\right), \quad \exp \left(C_{\sigma} T_{*}\right) \leq 2 .
$$


It is clear that $T_{*}>0$ is independent of $(\nu, \eta, \varepsilon)$. Then we have

$$
\begin{aligned}
& \sum_{k=1}^{\infty}\left(\Gamma^{k+1}\left(T_{*}\right)+\varepsilon a_{1} \int_{0}^{T_{*}}\left(v^{2}\left\|\nabla \bar{u}^{k+1}\right\|_{1}^{2}+\left|h^{k+1} \nabla \bar{u}^{k+1}\right|_{2}^{2}+\left|h^{k+1} \nabla^{2} \bar{u}^{k+1}\right|_{2}^{2}\right) \mathrm{d} s\right) \\
& \quad \leq C<\infty
\end{aligned}
$$

It follows from

$$
\begin{aligned}
& \lim _{k \mapsto \infty}\left|\bar{h}^{k+1}\right|_{\infty} \leq C \lim _{k \mapsto \infty}\left|\bar{\psi}^{k+1}\right|_{2}^{\frac{1}{2}}\left|\nabla \bar{\psi}^{k+1}\right|_{2}^{\frac{1}{2}} \leq C \lim _{k \mapsto \infty}\left|\bar{\psi}^{k+1}\right|_{2}^{\frac{1}{2}}=0, \\
& \lim _{k \mapsto \infty}\left|\bar{\psi}^{k+1}\right|_{6} \leq C \lim _{k \mapsto \infty}\left|\bar{\psi}^{k+1}\right|_{\infty}^{\frac{2}{3}}\left|\bar{\psi}^{k+1}\right|_{2}^{\frac{1}{3}} \leq C \lim _{k \mapsto \infty}\left|\bar{\psi}^{k+1}\right|_{2}^{\frac{1}{3}}=0
\end{aligned}
$$

that the whole sequence $\left(h^{k}, \psi^{k}, c^{k}, u^{k}\right)$ converges to a limit $(h, \psi, c, u)$ in the following strong sense:

$$
\begin{gathered}
h^{k} \rightarrow h \text { in } L^{\infty}\left(\left[0, T_{*}\right] \times \mathbb{R}^{3}\right), \quad \psi^{k} \rightarrow \psi \text { in } L^{\infty}\left(\left[0, T_{*}\right] ; L^{6}\left(\mathbb{R}^{3}\right)\right), \\
\left(c^{k}, u^{k}\right) \rightarrow(c, u) \text { in } L^{\infty}\left(\left[0, T_{*}\right] ; H^{1}\left(\mathbb{R}^{3}\right)\right) .
\end{gathered}
$$

From the uniform estimates (4.31), we have the following weak/weak* convergence:

$$
\begin{array}{cl}
\psi^{k} \rightarrow \psi & \text { weakly* in } L^{\infty}\left(\left[0, T_{*}\right] ; D^{1} \cap D^{2}\right), \\
U^{k} \rightarrow U & \text { weakly* in } L^{\infty}\left(\left[0, T_{*}\right] ; H^{3}\right), \\
\nabla u^{k} \rightarrow \nabla u & \text { weakly in } L^{2}\left(\left[0, T_{*}\right] ; H^{3}\right),
\end{array}
$$

which, along with the weakly lower semicontinuity of norms, implies that $(h, \psi, c, u)$ satisfies

$$
\varepsilon\|\psi(t)\|_{D^{1} \cap D^{2}}^{2}+\|U(t)\|_{3}^{2}+\int_{0}^{t} \varepsilon \sum_{i=1}^{4} v^{2}\left|\nabla^{i} u\right|_{2}^{2} \mathrm{~d} s \leq C d_{0}^{2} .
$$

Then it follows from the above uniform estimates, the strong convergence in (4.46), and the weak convergence in (4.47) that

$$
h^{k} \nabla^{i} u^{k} \rightarrow h \nabla^{i} u \quad \text { weakly in } L^{2}\left(\left[0, T_{*}\right] ; L^{2}\right) \text { for } i=1,2,3,4,
$$

which, along with the weakly lower semicontinuity of norms again, implies that $(h, \psi, c, u)$ satisfies the uniform estimates (4.31). Moreover, it follows from (4.46)-(4.48) and the uniform estimates (4.31) that $(h, \psi, U)$ satisfies (4.32)-(4.33) in the sense of distributions, respectively, with the following regularity:

$$
\begin{gathered}
h \in L^{\infty}\left(\left[0, T_{*}\right] \times \mathbb{R}^{3}\right), \quad \psi \in L^{\infty}\left(\left[0, T_{*}\right] ; H^{2}\right), \\
c \in L^{\infty}\left(\left[0, T_{*}\right] ; H^{3}\right), \quad u \in L^{\infty}\left(\left[0, T_{*}\right] ; H^{3}\right) \cap L^{2}\left(\left[0, T_{*}\right] ; H^{4}\right) .
\end{gathered}
$$

Finally, we need to verify the following relation:

$$
\psi^{\varepsilon}=\nabla h .
$$


Denote $\psi^{*}=\nabla h$ and $\bar{\psi}^{*}=\psi-\psi^{*}$. Then, using equations $(4.32)_{1}-(4.32)_{2}$, we have

$$
\left\{\begin{array}{l}
\bar{\psi}_{t}^{*}+\sum_{l=1}^{3} B_{l}(u) \partial_{l} \bar{\psi}^{*}+B(u) \bar{\psi}^{*}=0, \\
\left.\bar{\psi}^{*}\right|_{t=0}=0 \quad \text { in } \mathbb{R}^{3},
\end{array}\right.
$$

which, together with a standard energy method, implies

$$
\bar{\psi}^{*}=0 \quad \text { for }(t, x) \in\left[0, T_{*}\right] \times \mathbb{R}^{3} .
$$

Then the relation in (4.50) is verified.

2. Uniqueness. Let $\left(h_{1}, c_{1}, u_{1}\right)$ and $\left(h_{2}, c_{2}, u_{2}\right)$ be two solutions of the Cauchy problem (4.32) obtained in step 1 , and let $\psi_{i}=\nabla h_{i}, i=1,2$. Define

$$
\left\{\begin{array}{l}
\bar{h}:=h_{1}-h_{2}, \quad \bar{\psi}:=\psi_{1}-\psi_{2}=\nabla h_{1}-\nabla h_{2}, \\
\bar{c}:=c_{1}-c_{2}, \quad \bar{u}:=u_{1}-u_{2}, \quad \bar{U}:=U_{1}-U_{2} .
\end{array}\right.
$$

Then $(\bar{h}, \bar{\psi}, \bar{c}, \bar{u})$ satisfies the following problem:

$$
\left\{\begin{array}{l}
\bar{h}_{t}+u_{1} \cdot \nabla \bar{h}+\bar{u} \cdot \psi_{2}+\frac{\delta-1}{2}\left(\bar{h} \operatorname{div} u_{2}+h_{1} \operatorname{div} \bar{u}\right)=0 \\
\bar{\psi}_{t}+\sum_{l=1}^{3} B_{l}\left(u_{1}\right) \partial_{l} \bar{\psi}+B\left(u_{1}\right) \bar{\psi}+\frac{\delta-1}{2}\left(\bar{h} \nabla \operatorname{div} u_{2}+h_{1} \nabla \operatorname{div} \bar{u}\right) \\
=-\sum_{l=1}^{3} B_{l}(\bar{u}) \partial_{l} \psi_{2}-B(\bar{u}) \psi_{2} \\
A_{0} \bar{U}_{t}+\sum_{l=1}^{3} A_{l}\left(U_{1}\right) \partial_{l} \bar{U}+\varepsilon F\left(v, h_{1}, \bar{u}\right) \\
=-\sum_{l=1}^{3} A_{l}(\bar{U}) \partial_{l} U_{2}-\varepsilon\left(F\left(v, h_{1}, u_{2}\right)-F\left(v, h_{2}, u_{2}\right)\right) \\
\quad+\varepsilon\left(G\left(h_{1}, \bar{\psi}, u_{1}\right)+G\left(h_{1}, \psi_{2}, \bar{u}\right)+G\left(\bar{h}, \psi_{2}, u_{2}\right)\right) \\
\left.(\bar{h}, \bar{\psi}, \bar{U})\right|_{t=0}=(0,0,0) \quad \text { for } x \in \mathbb{R}^{3} .
\end{array}\right.
$$

Denote

$$
\Phi(t)=\varepsilon\left(|\bar{h}(t)|_{2}^{2}+|\bar{\psi}(t)|_{2}^{2}\right)+\|(\bar{c}, \bar{u})(t)\|_{1}^{2} .
$$

Similarly to the derivation of (4.39)-(4.40) and (4.45), we can show

$$
\frac{\mathrm{d}}{\mathrm{d} t} \Phi(t)+\varepsilon\left(\left|h_{1} \nabla \bar{u}(t)\right|_{2}^{2}+\left|h_{1} \nabla^{2} \bar{u}(t)\right|_{2}^{2}+v^{2}\|\nabla \bar{u}(t)\|_{1}^{2}\right) \leq G(t) \Phi(t),
$$

where $\int_{0}^{t} G(s) \mathrm{d} s \leq C$ for $0 \leq t \leq T_{*}$. From the Gronwall inequality, we conclude that

$$
\bar{h}=\bar{\psi}=\bar{c}=\bar{u}=0,
$$

which gives the desired uniqueness. 
3. Time continuity. The time continuity follows easily from the same procedure as in Lemma 4.1.

This completes the proof.

Remark 4.2. For completeness, we now give the proof of Lemma 4.4, which is quite direct.

Proof of Lemma 4.4. We define $\chi_{R}(x)=\chi\left(\frac{x}{R}\right)$ with $\chi(x) \in C_{c}^{\infty}\left(\mathbb{R}^{3}\right)$ as a truncation function satisfying (2.6).

Denote $\bar{h}^{k+1, R}=\bar{h}^{k+1} \chi_{R}$. From (4.38) , we have

$$
\begin{gathered}
\bar{h}_{t}^{k+1, R}+u^{k} \cdot \nabla \bar{h}^{k+1, R}+\frac{\delta-1}{2}\left(\bar{h}^{k, R} \operatorname{div} u^{k-1}+h^{k} \operatorname{div} \bar{u}^{k} \chi_{R}\right) \\
=u^{k} \bar{h}^{k+1} \cdot \nabla \chi_{R}-\bar{u}^{k} \cdot \psi^{k} \chi_{R} .
\end{gathered}
$$

Then multiplying the above equation by $2 \bar{h}^{k+1, R}$ and integrating over $\mathbb{R}^{3}$ yield

$$
\begin{aligned}
\frac{\mathrm{d}}{\mathrm{d} t}\left|\bar{h}^{k+1, R}\right|_{2} \leq & C\left|\nabla u^{k}\right|_{\infty}\left|\bar{h}^{k+1, R}\right|_{2}+C\left(\left|\bar{h}^{k}\right|_{\infty}\left|\operatorname{div} u^{k-1}\right|_{2}+\left|h^{k}\right|_{\infty}\left|\operatorname{div} \bar{u}^{k}\right|_{2}\right) \\
& +C\left(\left|u^{k}\right|_{2}\left|\bar{h}^{k+1}\right|_{\infty}+\left|\bar{u}^{k}\right|_{2}\left|\psi^{k}\right|_{\infty}\right) \\
\leq & \widehat{C}\left|\bar{h}^{k+1, R}\right|_{2}+\widehat{C}
\end{aligned}
$$

where $\widehat{C}>0$ is a constant depending on the generic constant $C$ and $\left(d_{0}, d_{1}, \eta\right)$, but is independent of $R$. Then, using the Gronwall inequality, we have

$$
\left|\bar{h}^{k+1, R}(t)\right|_{2} \leq \widehat{C} \exp \left(\widehat{C} T^{* *}\right) \quad \text { for }(t, R) \in\left[0, T^{* *}\right] \times[0, \infty) .
$$

Then $\bar{h}^{k+1} \in L^{\infty}\left(\left[0, T^{* *}\right] ; L^{2}\left(\mathbb{R}^{3}\right)\right)$, which, along with $\bar{h}^{k+1}=h^{k+1}-h^{k}$ and

$$
\nabla h^{k}=\psi^{k} \in L^{\infty}\left(\left[0, T^{* *}\right] ; D^{1} \cap D^{2}\left(\mathbb{R}^{3}\right)\right),
$$

implies that $\bar{h}^{k+1} \in L^{\infty}\left(\left[0, T^{* *}\right] ; H^{3}\left(\mathbb{R}^{3}\right)\right)$.

\subsection{Passing to the limit as $v \rightarrow 0$}

Now we consider the following nonlinear problem for $(h, c, u)$ :

$$
\left\{\begin{array}{l}
h_{t}+u \cdot \nabla h+\frac{\delta-1}{2} h \operatorname{div} u=0, \\
A_{0} U_{t}+\sum_{j=1}^{3} A_{j}(U) \partial_{j} U=-\varepsilon F(h, u)+\varepsilon G(h, \psi, u), \\
\left.(h, c, u)\right|_{t=0}=\left(h_{0}, c_{0}, u_{0}\right)(x)=\left((A \gamma)^{-\frac{\iota}{2}}\left(c_{0}+\eta\right)^{\iota}, c_{0}, u_{0}\right)(x) \text { for } x \in \mathbb{R}^{3},
\end{array}\right.
$$

where $\psi=\nabla h$ satisfying equations (4.33). 
The main result in this subsection can be stated as follows:

Theorem 4.3. Let (2.1) hold, and let $(\eta, \varepsilon) \in(0,1] \times(0,1]$. Assume that the initial data $\left(c_{0}, u_{0}\right)$ satisfy (4.13). Then there exist $T_{*}>0$ independent of $(\eta, \varepsilon)$ and a unique strong solution (h, $c, u)$ of problem (4.56) in $\left[0, T_{*}\right] \times \mathbb{R}^{3}$ satisfying the uniform estimates (4.31),

$$
\begin{gathered}
h \in L^{\infty} \cap C\left(\left[0, T_{*}\right] \times \mathbb{R}^{3}\right), \quad \psi \in C\left(\left[0, T_{*}\right] ; H^{2}\right), \\
c \in C\left(\left[0, T_{*}\right] ; H^{3}\right), \quad u \in C\left(\left[0, T_{*}\right] ; H^{3}\right) \cap L^{2}\left(\left[0, T_{*}\right] ; H^{4}\right),
\end{gathered}
$$

and

$$
\frac{C}{2}\left(1+\left|c_{0}\right|_{\infty}\right)^{\iota} \leq h^{\eta, \varepsilon}(t, x) \leq C \eta^{\iota} \quad \text { for }(t, x) \in\left[0, T_{*}\right] \times \mathbb{R}^{3},
$$

where $C>0$ is a constant independent of $(\eta, \varepsilon)$.

Proof. We prove the existence, uniqueness, and time continuity in two steps.

1. Existence. First, from Theorem 4.2, for every $(v, \eta, \varepsilon) \in(0,1] \times(0,1] \times(0,1]$, there exist $T_{*}>0$ independent of $(v, \eta, \varepsilon)$ and a unique strong solution

$$
\left(h^{\nu, \eta, \varepsilon}, c^{\nu, \eta, \varepsilon}, u^{\nu, \eta, \varepsilon}\right)(t, x) \quad \text { in }\left[0, T_{*}\right] \times \mathbb{R}^{3}
$$

of problem (4.32) satisfying the estimates in (4.31), which are independent of $(v, \eta, \varepsilon)$. Moreover, applying the characteristic method and the standard energy estimates for the transport equations, and using equations $(4.32)_{1}-(4.32)_{2}$ for $h^{\nu, \eta, \varepsilon}$ and $\psi^{\nu, \eta, \varepsilon}$, we have

$$
\begin{aligned}
& \frac{C}{2}\left(1+\left|c_{0}\right|_{\infty}\right)^{\iota} \leq h^{v, \eta, \varepsilon}(t, x) \leq C \eta^{\iota} \text { for }(t, x) \in\left[0, T_{*}\right] \times \mathbb{R}^{3}, \\
& \left\|h^{\nu, \eta, \varepsilon}(t)\right\|_{D^{1}}+\left|h_{t}^{v, \eta, \varepsilon}(t)\right|_{2} \leq \widehat{C}\left(\eta, \alpha, \beta, \gamma, \delta, T_{*}, c_{0}, u_{0}\right) \quad \text { for } 0 \leq t \leq T_{*} .
\end{aligned}
$$

Then, by virtue of the uniform estimates in (4.31) independent of $(v, \eta, \varepsilon)$, the estimates in (4.58) independent of $(v, \varepsilon)$, and the compactness in Lemma A.2 (see [51]), we obtain that, for any $R>0$, there exists a subsequence of solutions (still denoted by) $\left(h^{\nu, \eta, \varepsilon}, c^{\nu, \eta, \varepsilon}, u^{\nu, \eta, \varepsilon}\right)$ converging to a limit $\left(h^{\eta, \varepsilon}, c^{\eta, \varepsilon}, u^{\eta, \varepsilon}\right)$ as $v \rightarrow 0$ in the following strong sense:

$$
\left(h^{\nu, \eta, \varepsilon}, c^{\nu, \eta, \varepsilon}, u^{\nu, \eta, \varepsilon}\right) \rightarrow\left(h^{\eta, \varepsilon}, c^{\eta, \varepsilon}, u^{\eta, \varepsilon}\right) \quad \text { in } C\left(\left[0, T_{*}\right] ; H^{2}\left(B_{R}\right)\right)
$$

for any finite constant $R>0$, where $B_{R}$ is a ball centered at the origin with radius $R$.

Again, it follows from the uniform estimates in (4.31) and (4.58) that there exists a further subsequence (of the subsequence chosen above) of solutions (still denoted by) $\left(h^{\nu, \eta, \varepsilon}, c^{\nu, \eta, \varepsilon}, u^{\nu, \eta, \varepsilon}\right)$ converging to $\left(h^{\eta, \varepsilon}, c^{\eta, \varepsilon}, u^{\eta, \varepsilon}\right)$ as $\nu \rightarrow 0$ in the following sense:

$$
\begin{aligned}
\left(c^{\nu, \eta, \varepsilon}, u^{\nu, \eta, \varepsilon}\right) & \rightarrow\left(c^{\eta, \varepsilon}, u^{\eta, \varepsilon}\right) & & \text { weakly* in } L^{\infty}\left(\left[0, T_{*}\right] ; H^{3}\right), \\
h^{\nu, \eta, \varepsilon} & \rightarrow h^{\eta, \varepsilon} & & \text { weakly* in } L^{\infty}\left(\left[0, T_{*}\right] \times \mathbb{R}^{3}\right), \\
\psi^{\nu, \eta, \varepsilon}=\nabla h^{\nu, \eta, \varepsilon} & \rightarrow \psi^{\eta, \varepsilon} & & \text { weakly* in } L^{\infty}\left(\left[0, T_{*}\right] ; H^{2}\right), \\
\nabla u^{\nu, \eta, \varepsilon} & \rightarrow \nabla u^{\eta, \varepsilon} & & \text { weakly in } L^{2}\left(\left[0, T_{*}\right] ; H^{3}\right),
\end{aligned}
$$


which, along with the lower semicontinuity of weak/weak* convergence of the norms, implies that $\left(h^{\eta, \varepsilon}, \psi^{\eta, \varepsilon}, c^{\eta, \varepsilon}, u^{\eta, \varepsilon}\right)$ also satisfies the corresponding estimates in (4.31) and (4.58), except those estimates on $h^{\eta, \varepsilon} \nabla^{i} u^{\eta, \varepsilon}$ for $i=1,2,3,4$.

Together with the uniform estimates on $\left(h^{\eta, \varepsilon}, u^{\eta, \varepsilon}\right)$ obtained above, the strong convergence in (4.59), and the weak/weak* convergence in (4.60), we obtain that, for $i=$ $1,2,3,4$,

$$
h^{\nu, \varepsilon, \eta} \nabla^{i} u^{\nu, \eta, \varepsilon} \rightarrow h^{\eta, \varepsilon} \nabla^{i} u^{\eta, \varepsilon} \quad \text { weakly in } L^{2}\left(\left[0, T_{*}\right] ; L^{2}\right),
$$

which, along with the lower semicontinuity of weak convergence again, implies that $\left(h^{\eta, \varepsilon}, u^{\eta, \varepsilon}\right)$ also satisfies the uniform estimates on $h^{\eta, \varepsilon} \nabla^{i} u^{\eta, \varepsilon}$ for $i=1,2,3,4$.

Now we show that $\left(h^{\eta, \varepsilon}, c^{\eta, \varepsilon}, u^{\eta, \varepsilon}\right)$ is a weak solution of problem (4.56) in the sense of distributions. First, multiplying the equations for the time evolution of $u$ in (4.56) by a test function $w(t, x)=\left(w^{1}, w^{2}, w^{3}\right) \in C_{c}^{\infty}\left(\left[0, T_{*}\right) \times \mathbb{R}^{3}\right)$ on both sides and integrating over $\left[0, T_{*}\right] \times \mathbb{R}^{3}$, we have

$$
\begin{aligned}
\int_{0}^{T_{*}} & \int_{\mathbb{R}^{3}}\left(u^{v, \eta, \varepsilon} \cdot w_{t}-\left(u^{\nu, \eta, \varepsilon} \cdot \nabla\right) u^{v, \eta, \varepsilon} \cdot w+\frac{1}{\gamma-1}\left(c^{\nu, \eta, \varepsilon}\right)^{2} \operatorname{div} w\right) \mathrm{d} x \mathrm{~d} t \\
& +\int_{\mathbb{R}^{3}} u_{0}(x) \cdot w(0, x) \mathrm{d} x \\
\quad= & \int_{0}^{T_{*}} \int \varepsilon\left(\left(\left(h^{\nu, \eta, \varepsilon}\right)^{2}+v^{2}\right) L u^{\nu, \eta, \varepsilon}-\frac{2 \delta}{\delta-1} h^{\nu, \eta, \varepsilon} \psi^{\nu, \eta, \varepsilon} \cdot Q\left(u^{\nu, \eta, \varepsilon}\right)\right) \cdot w \mathrm{~d} x \mathrm{~d} t .
\end{aligned}
$$

Combining the uniform estimates obtained above with the strong convergence in (4.59) and the weak convergence in (4.60)-(4.61), and letting $v \rightarrow 0$ in (4.62), we obtain

$$
\begin{aligned}
\int_{0}^{T_{*}} & \int_{\mathbb{R}^{3}}\left(u^{\eta, \varepsilon} \cdot w_{t}-\left(u^{\eta, \varepsilon} \cdot \nabla\right) u^{\eta, \varepsilon} \cdot w+\frac{1}{\gamma-1}\left(c^{\eta, \varepsilon}\right)^{2} \operatorname{div} w\right) \mathrm{d} x \mathrm{~d} t \\
& +\int_{\mathbb{R}^{3}} u_{0}(x) \cdot w(0, x) \mathrm{d} x \\
= & \int_{0}^{T_{*}} \int_{\mathbb{R}^{3}} \varepsilon\left(\left(h^{\eta, \varepsilon}\right)^{2} L u^{\eta, \varepsilon}-\frac{2 \delta}{\delta-1} h^{\eta, \varepsilon} \psi^{\eta, \varepsilon} \cdot Q\left(u^{\eta, \varepsilon}\right)\right) \cdot w \mathrm{~d} x \mathrm{~d} t .
\end{aligned}
$$

Similarly, we can use the same argument to show that $\left(h^{\eta, \varepsilon}, c^{\eta, \varepsilon}, u^{\eta, \varepsilon}\right)$ satisfies the other equations in (4.56) and the corresponding initial data in the sense of distributions. Thus, $\left(h^{\eta, \varepsilon}, c^{\eta, \varepsilon}, u^{\eta, \varepsilon}\right)$ is a weak solution of problem (4.56) in the sense of distributions satisfying the following regularity:

$$
\begin{gathered}
h^{\eta, \varepsilon} \in\left(L^{\infty} \cap C\right)\left(\left[0, T_{*}\right] \times \mathbb{R}^{3}\right), \quad \psi^{\eta, \varepsilon} \in L^{\infty}\left(\left[0, T_{*}\right] ; H^{2}\right), \\
c^{\eta, \varepsilon} \in L^{\infty}\left(\left[0, T_{*}\right] ; H^{3}\right), \quad u^{\eta, \varepsilon} \in L^{\infty}\left(\left[0, T_{*}\right] ; H^{3}\right) \cap L^{2}\left(\left[0, T_{*}\right] ; H^{4}\right) .
\end{gathered}
$$

Therefore, this solution $\left(h^{\eta, \varepsilon}, c^{\eta, \varepsilon}, u^{\eta, \varepsilon}\right)$ of problem (4.56) is actually a strong solution. The relation $\psi^{\eta, \varepsilon}=\nabla h^{\eta, \varepsilon}$ can be verified by the same argument used in the proof of (4.50). 
2. Uniqueness and time continuity. Owing to the lower bound estimate of $h^{\eta, \varepsilon}$,

$$
h^{\eta, \varepsilon}(t, x) \geq \frac{C}{2}\left(1+\left|c_{0}\right|_{\infty}\right)^{\iota} \quad \text { for }(t, x) \in\left[0, T_{*}\right] \times \mathbb{R}^{3},
$$

the uniqueness and time continuity of the strong solutions obtained above can be proved via a similar argument to Theorem 4.2; hence we omit its details.

\subsection{Passing to the limit as $\eta \rightarrow 0$}

Based on the conclusions obtained in the above subsections, we are now ready to prove Theorem 4.1 .

Proof of Theorem 4.1. We divide the proof into three steps.

1. Existence. As in Section 4.2, we define constants $d_{i}, i=0,1$. For any $\eta \in(0,1)$, set

$$
h_{0}^{\eta}=(A \gamma)^{-\frac{\iota}{2}}\left(c_{0}+\eta\right)^{\iota}, \quad \psi_{0}^{\eta}=(A \gamma)^{-\frac{\iota}{2}} \nabla\left(c_{0}+\eta\right)^{\iota} .
$$

Then there exists $\eta_{1}>0$ such that, if $0<\eta<\eta_{1}$,

$$
\eta+\varepsilon^{\frac{1}{2}}\left\|\psi_{0}^{\eta}\right\|_{D^{1} \cap D^{2}}+\left|\left(h_{0}^{\eta}\right)^{-1}\right|_{\infty}+\varepsilon^{\frac{1}{4}}\left|\nabla c_{0}^{\frac{l}{2}}\right|_{4}+\left\|\left(c_{0}, u_{0}\right)\right\|_{3} \leq \bar{d}_{0},
$$

where we have used the fact that $\varepsilon^{\frac{1}{4}} \nabla c_{0}^{\frac{\ell}{2}} \in L^{4}$, and $\bar{d}_{0}$ is a positive constant independent of $\eta$. Therefore, taking $\left(h_{0}^{\eta}, c_{0}, u_{0}\right)$ as the initial data, problem (4.56) admits a unique strong solution $\left(h^{\eta, \varepsilon}, c^{\eta, \varepsilon}, u^{\eta, \varepsilon}\right)$ in $\left[0, T_{*}\right] \times \mathbb{R}^{3}$ satisfying the local estimates in (4.31) with $d_{0}$ replaced by $\bar{d}_{0}$, and the life span $T_{*}$ is also independent of $(\eta, \varepsilon)$. Moreover, we also know that

$$
h^{\eta, \varepsilon} \geq \frac{C}{2}\left(1+\left|c_{0}\right|_{\infty}\right)^{\iota} \quad \text { for }(t, x) \in\left[0, T_{*}\right] \times \mathbb{R}^{3},
$$

where $C>0$ is a constant independent of $(\eta, \varepsilon)$.

We first state the following lemma:

Lemma 4.5. For any $R_{0}>0$ and $(\eta, \varepsilon) \in(0,1] \times(0,1]$, there exists a constant $b_{R_{0}}>0$ such that

$$
h^{\eta, \varepsilon}(t, x) \leq b_{R_{0}} \quad \text { for any }(t, x) \in\left[0, T_{*}\right] \times B_{R_{0}},
$$

where $b_{R_{0}}>0$ is a constant independent of $(\eta, \varepsilon)$.

Proof. It suffices to consider the case when $R_{0}$ is sufficiently large.

First, from the initial assumptions on $c_{0}$,

$$
c_{0}(x)>0, \quad c_{0} \in H^{3},
$$

we obtain that the initial vacuum occurs only in the far-field. Then, for every $R^{\prime}>2$, there exists a constant $C_{R^{\prime}}$ independent of $(\eta, \varepsilon)$ such that

$$
c_{0}^{\eta}(x) \geq C_{R^{\prime}}+\eta>0 \quad \text { for any } x \in B_{R^{\prime}},
$$


which implies

$$
h_{0}^{\eta}(x) \leq(A \gamma)^{-\frac{\iota}{2}}\left(C_{R^{\prime}}+2 \eta\right)^{\iota} \leq(A \gamma)^{-\frac{\iota}{2}} C_{R^{\prime}}^{\iota} \quad \text { for any } x \in B_{R^{\prime}} .
$$

Second, let $x^{\eta, \varepsilon}\left(t ; x_{0}\right)$ be the particle path starting from $x_{0}$ at $t=0$ :

$$
\frac{\mathrm{d}}{\mathrm{d} t} x^{\eta, \varepsilon}\left(t ; x_{0}\right)=u^{\eta, \varepsilon}\left(t, x\left(t ; x_{0}\right)\right), \quad x^{\eta, \varepsilon}\left(0 ; x_{0}\right)=x_{0} .
$$

Denote by $B\left(t, R^{\prime}\right)$ the closed regions that are the images of $B_{R^{\prime}}$ under the flow map (4.67):

$$
B\left(t, R^{\prime}\right)=\left\{x^{\eta, \varepsilon}\left(t ; x_{0}\right): x_{0} \in B_{R^{\prime}}\right\} .
$$

From equation (4.56) 1 , we have

$$
h^{\eta, \varepsilon}(t, x)=h_{0}^{\eta}\left(x_{0}\right) \exp \left(-\frac{\delta-1}{2} \int_{0}^{t} \operatorname{div} u^{\eta, \varepsilon}\left(s, x^{\eta, \varepsilon}\left(s ; x_{0}\right)\right) \mathrm{d} s\right) .
$$

According to (4.31), for $0 \leq t \leq T_{*}$,

$$
\begin{aligned}
& \int_{0}^{t}\left|\operatorname{div} u^{\eta, \varepsilon}\left(t, x^{\eta, \varepsilon}\left(t ; x_{0}\right)\right)\right| \mathrm{d} s \leq \int_{0}^{t}\left|\nabla u^{\eta, \varepsilon}\right|_{\infty} \mathrm{d} s \\
& \leq \int_{0}^{t}\left\|\nabla u^{\eta, \varepsilon}\right\|_{2} \mathrm{~d} s \leq t^{\frac{1}{2}}\left(\int_{0}^{t}\left\|\nabla u^{\eta, \varepsilon}\right\|_{2}^{2} \mathrm{~d} s\right)^{\frac{1}{2}} \leq d_{1} T_{*}^{\frac{1}{2}} .
\end{aligned}
$$

Thus, by (4.66) and (4.68)-(4.69), we see that, for $0 \leq t \leq T_{*}$,

$$
h^{\eta, \varepsilon}(t, x) \leq(A \gamma)^{-\frac{\iota}{2}} C^{*} C_{R^{\prime}}^{\iota} \quad \text { for any } x \in B\left(t, R^{\prime}\right),
$$

where $C^{*}=\exp \left(\frac{1}{2} d_{1} T_{*}^{\frac{1}{2}}\right)$.

Finally, from problem (4.67) and the estimates in (4.31), we have

$$
\left|x_{0}-x\right|=\left|x_{0}-x^{\eta, \varepsilon}\left(t ; x_{0}\right)\right| \leq \int_{0}^{t}\left|u^{\eta, \varepsilon}\left(\tau, x^{\eta, \varepsilon}\left(\tau ; x_{0}\right)\right)\right| \mathrm{d} \tau \leq d_{1} t \leq 1 \leq \frac{R^{\prime}}{2}
$$

for all $(t, x) \in\left[0, T_{*}\right] \times B_{R}$, which implies that $B_{R^{\prime} / 2} \subset B\left(t, R^{\prime}\right)$. Therefore, we choose

$$
R^{\prime}=2 R_{0}, \quad b_{R_{0}}=(A \gamma)^{-\frac{\iota}{2}} C^{*} C_{R^{\prime}}^{\iota}
$$

to complete the proof.

Then, for any $R>0$, it follows from Lemmas 4.5 and A.2 that there exists a subsequence (still denoted by) $\left(h^{\eta, \varepsilon}, \psi^{\eta, \varepsilon}, c^{\eta, \varepsilon}, u^{\eta, \varepsilon}\right)$ such that

$$
\begin{aligned}
\left(h^{\eta, \varepsilon}, c^{\eta, \varepsilon}, u^{\eta, \varepsilon}\right) & \rightarrow\left(h^{\varepsilon}, c^{\varepsilon}, u^{\varepsilon}\right) & & \text { in } C\left(\left[0, T_{*}\right] ; H^{2}\left(B_{R}\right)\right), \\
\psi^{\eta, \varepsilon} & \rightarrow \psi^{\varepsilon} & & \text { in } C\left(\left[0, T_{*}\right] ; H^{1}\left(B_{R}\right)\right) .
\end{aligned}
$$

This, together with Lemma 4.5, yields the following lemma: 
Lemma 4.6. For any $R_{0}>0$ and $\varepsilon \in(0,1]$, there exists a constant $b_{R_{0}}>0$ such that

$$
h^{\varepsilon}(t, x) \leq b_{R_{0}} \quad \text { for any }(t, x) \in\left[0, T_{*}\right] \times B_{R_{0}},
$$

where $b_{R_{0}}$ is independent of $\varepsilon$.

Next, note that estimates (4.31) are independent of $(\eta, \varepsilon)$. Then there exists a subsequence (still denoted by) $\left(h^{\eta, \varepsilon}, \psi^{\eta, \varepsilon}, c^{\eta, \varepsilon}, u^{\eta, \varepsilon}\right)$ converging to a limit $\left(h^{\varepsilon}, \psi^{\varepsilon}, c^{\varepsilon}, u^{\varepsilon}\right)$ in the weak or weak* sense:

$$
\begin{aligned}
\left(c^{\eta, \varepsilon}, u^{\eta, \varepsilon}\right) & \rightarrow\left(c^{\varepsilon}, u^{\varepsilon}\right) & & \text { weakly* in } L^{\infty}\left(\left[0, T_{*}\right] ; H^{3}\right), \\
\psi^{\eta, \varepsilon} & \rightarrow \psi^{\varepsilon} & & \text { weakly* in } L^{\infty}\left(\left[0, T_{*}\right] ; D^{1} \cap D^{2}\right), \\
u^{\eta, \varepsilon} & \rightarrow u^{\varepsilon} & & \text { weakly in } L^{2}\left(\left[0, T_{*}\right] ; H^{4}\right) .
\end{aligned}
$$

From the lower semicontinuity of the weak convergence, $\left(h^{\varepsilon}, \psi^{\varepsilon}, c^{\varepsilon}, u^{\varepsilon}\right)$ also satisfies the corresponding estimates (4.31), except those of $h^{\varepsilon} \nabla^{i} u^{\varepsilon}$ for $i=1,2,3,4$.

Together with the uniform estimates of $\left(h^{\varepsilon}, \psi^{\varepsilon}, c^{\varepsilon}, u^{\varepsilon}\right)$ obtained above, the strong convergence in (4.71), and the weak or weak* convergence in (4.73), we obtain that, for $i=1,2,3,4$,

$$
h^{\varepsilon, \eta} \nabla^{i} u^{\varepsilon, \eta} \rightarrow h^{\varepsilon} \nabla^{i} u^{\varepsilon} \quad \text { weakly in } L^{2}\left(\left[0, T_{*}\right] ; L^{2}\right),
$$

which, along with the lower semicontinuity of weak or weak* convergence again, implies that $\left(h^{\varepsilon}, u^{\varepsilon}\right)$ also satisfies the uniform estimates of $h^{\varepsilon} \nabla^{i} u^{\varepsilon}$ for $i=1,2,3,4$.

Thus, it is easy to show that $\left(h^{\varepsilon}, c^{\varepsilon}, u^{\varepsilon}\right)$ solves the following Cauchy problem in the sense of distributions:

$$
\left\{\begin{array}{l}
h_{t}^{\varepsilon}+u^{\varepsilon} \cdot \nabla h^{\varepsilon}+\frac{\delta-1}{2} h^{\varepsilon} \operatorname{div} u^{\varepsilon}=0 \\
A_{0} U_{t}^{\varepsilon}+\sum_{j=1}^{3} A_{j}\left(U^{\varepsilon}\right) \partial_{j} U^{\varepsilon}=-\varepsilon F\left(h^{\varepsilon}, u^{\varepsilon}\right)+\varepsilon G\left(h^{\varepsilon}, \psi^{\varepsilon}, u^{\varepsilon}\right),
\end{array}\right.
$$

with the initial data

$$
\left.\left(h^{\varepsilon}, c^{\varepsilon}, u^{\varepsilon}\right)\right|_{t=0}=\left(h_{0}, c_{0}, u_{0}\right)(x)=\left(\rho_{0}^{\frac{\delta-1}{2}}, \sqrt{A \gamma} \rho_{0}^{\frac{\gamma-1}{2}}, u_{0}\right)(x) \text { for } x \in \mathbb{R}^{3},
$$

so that

$$
\left(\rho_{0}, u_{0}\right) \rightarrow(0,0) \quad \text { as }|x| \rightarrow \infty .
$$

Moreover, in this step, even though the vacuum appears in the far-field, $\psi^{\varepsilon}$ satisfies $\partial_{i}\left(\psi^{\varepsilon}\right)^{(j)}=\partial_{j}\left(\psi^{\varepsilon}\right)^{(i)}, i, j=1,2,3$, and the equation

$$
\psi_{t}^{\varepsilon}+\sum_{l=1}^{3} B_{l}\left(u^{\varepsilon}\right) \partial_{l} \psi^{\varepsilon}+B\left(u^{\varepsilon}\right) \psi^{\varepsilon}+\frac{\delta-1}{2} h^{\varepsilon} \nabla \operatorname{div} u^{\varepsilon}=0
$$

in the sense of distributions. 
Finally, we need to verify the relations

$$
\psi^{\varepsilon}=\nabla h^{\varepsilon}, \quad h^{\varepsilon}=(A \gamma)^{-\frac{\iota}{2}}\left(c^{\varepsilon}\right)^{\iota} .
$$

The first relation can be verified by the same argument used in the proof of (4.50). For the second, we denote

$$
h^{*}=(A \gamma)^{-\frac{\iota}{2}}\left(c^{\varepsilon}\right)^{\iota}, \quad \bar{h}^{*}=h^{*}-h^{\varepsilon} .
$$

Then using equations $(4.75)_{1}$ and $(4.75)_{3}$ leads to

$$
\left\{\begin{array}{l}
\bar{h}_{t}^{*}+u^{\varepsilon} \cdot \nabla \bar{h}^{*}+\frac{\delta-1}{2} \bar{h}^{*} \operatorname{div} u^{\varepsilon}=0, \\
\left.\bar{h}^{*}\right|_{t=0}=0 \quad \text { in } \mathbb{R}^{3}
\end{array}\right.
$$

which, together with the standard energy method, implies

$$
\bar{h}^{*}=0 \quad \text { for }(t, x) \in\left[0, T_{*}\right] \times \mathbb{R}^{3} .
$$

Then the relations in (4.79) have been verified.

2. Uniqueness. Owing to the lower bound estimate of $h^{\varepsilon}$,

$$
h^{\varepsilon}(t, x) \geq \frac{C}{2}\left(1+\left|c_{0}\right|_{\infty}\right)^{\iota} \quad \text { for }(t, x) \in\left[0, T_{*}\right] \times \mathbb{R}^{3},
$$

the uniqueness of the strong solutions obtained above can be proved via a similar argument to that used in the proof of Theorem 4.2, and hence we omit the details.

3. Time continuity. First, from the uniform estimates in (4.31) and the classical Sobolev embedding theorem, we obtain that, for any $s^{\prime} \in(0,3)$ and $s^{\prime \prime} \in(0,1)$,

$$
c^{\varepsilon} \in C\left(\left[0, T_{*}\right] ; H^{s^{\prime}} \cap \text { weak- } H^{3}\right), \quad \nabla \psi^{\varepsilon} \in C\left(\left[0, T_{*}\right] ; L^{2} \cap \text { weak- } H^{s^{\prime \prime}}\right) .
$$

Using similar arguments to the proofs of Lemmas 4.2-4.3 yields

$$
\lim _{t \rightarrow 0} \sup \left\|c^{\varepsilon}(t)\right\|_{3} \leq\left\|c_{0}\right\|_{3}, \quad \lim _{t \rightarrow 0} \sup \left\|\psi^{\varepsilon}(t)\right\|_{D^{1} \cap D^{2}} \leq\left\|\psi_{0}\right\|_{D^{1} \cap D^{2}},
$$

which, together with Lemma A.6 and (4.81), implies that $\left(c^{\varepsilon}, \psi^{\varepsilon}\right)$ is right-continuous at $t=0$ in $H^{3}$ and $D^{1} \cap D^{2}$, respectively. The time reversibility of the equations in (4.75) for $\left(c^{\varepsilon}, \psi^{\varepsilon}\right)$ yields

$$
c^{\varepsilon} \in C\left(\left[0, T_{*}\right] ; H^{3}\right), \quad \psi^{\varepsilon} \in C\left(\left[0, T_{*}\right] ; D^{1} \cap D^{2}\right) .
$$

For velocity $u^{\varepsilon}$, from the equations in (4.75) for $u^{\varepsilon}$, Lemma 4.6, and the classical Sobolev embedding theorem, we conclude that $u^{\varepsilon} \in C\left(\left[0, T_{*}\right] ; H_{\mathrm{loc}}^{3}\left(\mathbb{R}^{3}\right)\right)$. 


\section{Proof of Theorem 2.1}

Based on the conclusion of Theorem 4.1, we are ready to establish the local-in-time wellposedness for the regular solutions of the original Cauchy problem (1.1)-(1.5) with (1.7)(1.8) shown in Theorem 2.1. For simplicity, in this section we denote $\left(\rho^{\varepsilon}, u^{\varepsilon}, c^{\varepsilon}, \psi^{\varepsilon}, h^{\varepsilon}\right)$ as $(\rho, u, c, \psi, h)$ and $\left(\rho_{0}^{\varepsilon}, u_{0}^{\varepsilon}, c_{0}^{\varepsilon}, \psi_{0}^{\varepsilon}, h_{0}^{\varepsilon}\right)$ as $\left(\rho_{0}, u_{0}, c_{0}, \psi_{0}, h_{0}\right)$.

Proof of Theorem 2.1. We divide the proof into two steps.

1. Existence and uniform regularity. It follows from the initial assumptions (2.2) and Theorem 4.1 that there exists $T_{*}>0$ such that the Cauchy problem (3.8)-(3.10) has a unique strong solution $(\psi, c, u)=\left((A \gamma)^{-\frac{l}{2}} \nabla c^{\iota}, c, u\right)$ with the regularity properties (4.2) and the uniform estimates (4.3). Denote $\rho=a_{3} c^{\frac{2}{\gamma-1}}$ with $a_{3}=(A \gamma)^{-\frac{1}{\gamma-1}}$. Then we obtain that $\psi=\nabla \rho^{\frac{\delta-1}{2}}=\nabla h$ and

$$
0<\sqrt{A \gamma} \rho^{\frac{\gamma-1}{2}}=c \in C^{1}\left(\left[0, T_{*}\right] \times \mathbb{R}^{3}\right), \quad(u, \nabla u) \in C\left(\left(0, T_{*}\right] \times \Omega\right)
$$

for any bounded smooth domain $\Omega \subset \mathbb{R}^{3}$.

First, multiplying (3.3) 2 by $\frac{\partial \rho}{\partial c}=\frac{2 a_{3}}{\gamma-1} c^{\frac{3-\gamma}{\gamma-1}}$, we obtain the continuity equation in (1.1).

Then multiplying (3.3) 3 by $\rho=a_{3} c^{\frac{2}{\gamma-1}}$ leads to the momentum equations in (1.1).

Next we need to show that $(\rho, u)$ also satisfies

$$
\rho^{\frac{1-\delta}{2}} u_{t} \in L^{\infty}\left([0, T] ; H^{1}\right) \cap L^{2}\left([0, T] ; H^{2}\right), \quad \rho^{\frac{1-\delta}{2}} u \in C\left([0, T] ; H^{3}\right) .
$$

In fact, from the momentum equations $(1.1)_{2}$ and the positivity, $\rho(t, x)>0$ for $(t, x) \in$ $\left[0, T_{*}\right] \times \mathbb{R}^{3}$, we have

$$
\rho^{\frac{1-\delta}{2}}\left(u_{t}+u \cdot \nabla u+\frac{2 A \gamma}{\gamma-1} \rho^{\frac{\gamma-1}{2}} \nabla \rho^{\frac{\gamma-1}{2}}\right)+\varepsilon \rho^{\frac{\delta-1}{2}} L u=\frac{2 \delta \varepsilon}{\delta-1} \nabla \rho^{\frac{\delta-1}{2}} \cdot Q(u),
$$

which, along with the uniform estimates (4.3) and the classical Sobolev embedding theorem, implies (5.2).

Therefore, $(\rho, u)$ satisfies (1.1)-(1.5) with (1.7)-(1.8) in the sense of distributions, the regularity properties in Definition 2.1, and the uniform estimates in Theorem 2.1.

2. Conservation laws. First, we show that the solution $(\rho, u)$ obtained above still satisfies

$$
\rho \in L^{\infty}\left(\left[0, T_{*}\right] ; L^{1}\left(\mathbb{R}^{3}\right)\right) \text { if } \rho_{0} \in L^{1} .
$$

Let $f: \mathbb{R}^{+} \rightarrow \mathbb{R}^{+}$be a nonincreasing $C^{2}$ function satisfying

$$
f(s)= \begin{cases}1, & s \in\left[0, \frac{1}{2}\right] \\ e^{-s}, & s \geq 1\end{cases}
$$

Denote $f_{R}(x)=f\left(\frac{|x|}{R}\right)$. Using the regularity of the solution and the definition of $f$, we have

$$
\int\left(\rho+\left|\rho_{t}\right|+|\operatorname{div}(\rho u)|\right) f_{R}(x) \mathrm{d} x+\frac{1}{R} \int\left(\left|\rho u f^{\prime}\left(\frac{|x|}{R}\right)\right|+\rho|u| f\left(\frac{|x|}{R}\right)\right) \mathrm{d} x \leq C(R)
$$

for any fixed $R>1$, where $C(R)>0$ is a constant depending only on $R$. 
Next, since the continuity equation $(1.1)_{1}$ holds, we can multiply $(1.1)_{1}$ by $f_{R}(x)$ and integrate with respect to $x$ to obtain

$$
\frac{\mathrm{d}}{\mathrm{d} t} \int \rho f_{R}(x) \mathrm{d} x=-\int \operatorname{div}(\rho u) f_{R}(x) \mathrm{d} x .
$$

Then it follows from integration by parts and Hölder's inequality that

$$
\begin{aligned}
-\int \operatorname{div}(\rho u) f_{R}(x) \mathrm{d} x=\int \rho u \cdot \frac{x}{|x| R} f^{\prime}\left(\frac{|x|}{R}\right) \mathrm{d} x & \leq C \int \rho|u| f\left(\frac{|x|}{R}\right) \frac{1}{R} \mathrm{~d} x \\
& \leq C \frac{|u|_{\infty}}{R} \int \rho f_{R}(x) \mathrm{d} x
\end{aligned}
$$

which, along with (5.4) and Gronwall's inequality, implies

$$
\sup _{0 \leq t \leq T_{*}} \int \rho f_{R}(x) \mathrm{d} x \leq C \int \rho_{0} f_{R}(x) \mathrm{d} x+C \leq C,
$$

with $C$ independent of $R$. Note that $\rho f_{R}(x) \rightarrow \rho$ as $R \rightarrow \infty$ for all $x \in \mathbb{R}^{3}$. Thus, by Fatou's lemma, we have

$$
\sup _{0 \leq t \leq T_{*}} \int \rho \mathrm{d} x \leq \sup _{0 \leq t \leq T_{*}} \liminf _{R \rightarrow \infty} \int \rho f_{R}(x) \mathrm{d} x \leq C,
$$

where $C$ is a constant depending only on $(\alpha, \beta, \gamma, \delta)$ and the initial data $\left(\rho_{0}, u_{0}\right)$.

Then $(\rho, u)(t, x)$ in $\left[0, T_{*}\right] \times \mathbb{R}^{3}$ obtained above has finite mass $m(t)$ and finite energy $E(t)$. Indeed,

$$
E(t)=\int\left(\frac{1}{2} \rho|u|^{2}+\frac{p}{\gamma-1}\right)(t, x) \mathrm{d} x \leq C\left(|u|_{2}^{2}+|\rho|_{1}\right)<\infty .
$$

For the conservation laws, since $\rho u \in W^{1,1}\left(\mathbb{R}^{3}\right)$, we have

$$
\frac{\mathrm{d}}{\mathrm{d} t} m(t)=0
$$

On the other hand, multiplying equation $(1.1)_{2}$ by $u$ on both sides and integrating over $\mathbb{R}^{3}$, via integration by parts and the continuity equation, we have

$$
\frac{\mathrm{d}}{\mathrm{d} t} E(t)+\int \rho^{\delta}\left(\alpha|\nabla u|_{2}^{2}+(\alpha+\beta)|\operatorname{div} u|_{2}^{2}\right) \mathrm{d} x=0,
$$

where we have used the fact that $\rho u|u|^{2}, u p, \rho^{\delta} \nabla u \cdot u \in W^{1,1}\left(\mathbb{R}^{3}\right)$, and

$$
p_{t}+u \cdot \nabla p+p \operatorname{div} u=-(\gamma-1) p \operatorname{div} u \quad \text { in }\left[0, T_{*}\right] \times \mathbb{R}^{3} .
$$

This completes the proof. 


\section{Vanishing viscosity limit as $\varepsilon \rightarrow 0$}

In this section we establish the vanishing viscosity limit results stated in Theorem 2.2 and also give the proofs of Corollaries 2.1-2.2.

\subsection{Proof of Theorem 2.2}

We divide the proof into five steps.

1. First we denote by

$$
\left(\psi^{\varepsilon}, U^{\varepsilon}\right)=\left(\psi^{\varepsilon}, c^{\varepsilon}, u^{\varepsilon}\right)^{\top}=\left(\nabla\left(\rho^{\varepsilon}\right)^{\frac{\delta-1}{2}}, \sqrt{A \gamma}\left(\rho^{\varepsilon}\right)^{\frac{\gamma-1}{2}}, u^{\varepsilon}\right)^{\top}
$$

the solution of the problem

$$
\left\{\begin{array}{l}
\psi_{t}^{\varepsilon}+\sum_{l=1}^{3} B_{l}\left(u^{\varepsilon}\right) \partial_{l} \psi^{\varepsilon}+B\left(u^{\varepsilon}\right) \psi^{\varepsilon}+\frac{\delta-1}{2} h^{\varepsilon} \nabla \operatorname{div} u^{\varepsilon}=0 \\
A_{0} U_{t}^{\varepsilon}+\sum_{j=1}^{3} A_{j}\left(U^{\varepsilon}\right) \partial_{j} U^{\varepsilon}=-\varepsilon F\left(h^{\varepsilon}, u^{\varepsilon}\right)+\varepsilon G\left(h^{\varepsilon}, \psi^{\varepsilon}, u^{\varepsilon}\right), \\
\left.\left(\psi^{\varepsilon}, c^{\varepsilon}, u^{\varepsilon}\right)\right|_{t=0}=\left(\nabla\left(\rho_{0}^{\varepsilon}\right)^{\frac{\delta-1}{2}}, \sqrt{A \gamma}\left(\rho_{0}^{\varepsilon}\right)^{\frac{\gamma-1}{2}}, u_{0}^{\varepsilon}\right)(x) \text { for } x \in \mathbb{R}^{3},
\end{array}\right.
$$

where $h^{\varepsilon}=\left(\rho^{\varepsilon}\right)^{\frac{\delta-1}{2}}=(A \gamma)^{-\frac{\iota}{2}}\left(c^{\varepsilon}\right)^{\iota}$. From Theorem 4.1, there exists $T_{1}>0$ independent of $\varepsilon$ such that $\left(\psi^{\varepsilon}, U^{\varepsilon}\right)$ of $(6.1)$ satisfies

$$
\sup _{0 \leq t \leq T_{1}}\left(\left\|\left(c^{\varepsilon}, u^{\varepsilon}\right)(t, \cdot)\right\|_{3}^{2}+\varepsilon\left\|\psi^{\varepsilon}(t, \cdot)\right\|_{D^{1} \cap D^{2}}^{2}\right)+\varepsilon \int_{0}^{T_{1}} \sum_{i=1}^{4}\left|h^{\varepsilon} \nabla^{i} u^{\varepsilon}(t, \cdot) g\right|_{2}^{2} \mathrm{~d} t \leq C_{0}
$$

for some positive constant $C_{0}=C_{0}\left(\alpha, \beta, A, \gamma, \delta, c_{0}, \psi_{0}, u_{0}\right)$ that is independent of $\varepsilon$.

2. Notice that the initial data $\left(\rho_{0}^{\varepsilon}, u_{0}^{\varepsilon}\right)$ satisfy (2.2)-(2.3) and that there exists a vector function $\left(\rho_{0}(x), u_{0}(x)\right)$ defined in $\mathbb{R}^{3}$ such that

$$
\lim _{\varepsilon \rightarrow 0}\left|\left(c_{0}^{\varepsilon}-c_{0}, u_{0}^{\varepsilon}-u_{0}\right)\right|_{2}=0 .
$$

Then $\left(c_{0}, u_{0}\right) \in H^{3}\left(\mathbb{R}^{3}\right)$, due to the lower semicontinuity of the weak convergence.

Based on [43], regarding $\left(\rho_{0}, u_{0}\right)$ as the initial data, we denote by

$$
U=(c, u)=\left(\sqrt{A \gamma} \rho^{\frac{\gamma-1}{2}}, u\right)
$$

the regular solution of the Cauchy problem (1.6) with (1.13), which can be written as the symmetric system

$$
\left\{\begin{array}{l}
A_{0} U_{t}+\sum_{j=1}^{3} A_{j}(U) \partial_{j} U=0, \\
U(0, x)=U_{0}=\left(\sqrt{A \gamma} \rho_{0}^{\frac{\gamma-1}{2}}, u_{0}\right) .
\end{array}\right.
$$


From [43] (see Theorem 1.1), we know that there exists $T_{2}$ such that there is a unique regular solution $U$ of problem (6.3) satisfying

$$
\sup _{0 \leq t \leq T_{2}}\|U(t, \cdot)\|_{3}^{2} \leq C
$$

for some positive constant $C=C\left(A, \gamma, c_{0}, u_{0}\right)$.

3. Denote $T_{*}=\min \left\{T_{1}, T_{2}\right\}>0$. Then, for any bounded smooth domain $\Omega$, due to Lemma 4.6 and the Aubin-Lions lemma (see [51]) (i.e., Lemma A.2), there exist a subsequence (still denoted by) $\left(c^{\varepsilon}, u^{\varepsilon}\right)$ and a limit vector function $\left(c^{*}, u^{*}\right)$ satisfying

$$
\left(c^{\varepsilon}, u^{\varepsilon}\right) \rightarrow\left(c^{*}, u^{*}\right) \quad \text { in } C\left(\left[0, T_{*}\right] ; H^{2}(\Omega)\right) .
$$

Note that estimates (4.31) are independent of $\varepsilon$. Then there exists a subsequence (still denoted by) $\left(c^{\varepsilon}, u^{\varepsilon}\right)$ converging to the limit function $\left(c^{*}, u^{*}\right)$ in the weak or weak* sense:

$$
\left(c^{\varepsilon}, u^{\varepsilon}\right) \rightarrow\left(c^{*}, u^{*}\right) \quad \text { weakly* in } L^{\infty}\left(\left[0, T_{*}\right] ; H^{3}\right) .
$$

From the lower semicontinuity of the norms in the weak convergence, $\left(c^{*}, u^{*}\right)$ also satisfies the corresponding estimates (4.31), except those on $h$ and $\psi$.

4. Now we show that $\left(c^{*}, u^{*}\right)$ is a weak solution of (6.3) in the sense of distributions. First, multiplying the equations in (6.1) for the fluid velocity $u^{\varepsilon}$ by a test function $w(t, x)=$ $\left(w^{1}, w^{2}, w^{3}\right) \in C_{c}^{\infty}\left(\left[0, T^{*}\right) \times \mathbb{R}^{3}\right)$ on both sides and integrating over $\left[0, T^{*}\right] \times \mathbb{R}^{3}$ yield

$$
\begin{gathered}
\int_{0}^{T^{*}} \int\left(u^{\varepsilon} \cdot w_{t}-\left(u^{\varepsilon} \cdot \nabla\right) u^{\varepsilon} \cdot w+\frac{1}{\gamma-1}\left(c^{\varepsilon}\right)^{2} \operatorname{div} w\right) \mathrm{d} x \mathrm{~d} t+\int u_{0}^{\varepsilon}(x) \cdot w(0, x) \mathrm{d} x \\
=\int_{0}^{T^{*}} \int \varepsilon\left(\left(h^{\varepsilon}\right)^{2} L u^{\varepsilon}-\frac{2 \delta}{\delta-1} h^{\varepsilon} \psi^{\varepsilon} \cdot Q\left(u^{\varepsilon}\right)\right) \cdot w \mathrm{~d} x \mathrm{~d} t
\end{gathered}
$$

Combining the uniform estimates obtained above, the strong convergence in (6.5), the weak convergence in (6.6), and (2.7), and letting $\varepsilon \rightarrow 0$ in (6.7), we have

$\int_{0}^{T^{*}} \int\left(u^{*} \cdot w_{t}-\left(u^{*} \cdot \nabla\right) u^{*} \cdot w+\frac{1}{\gamma-1}\left(c^{*}\right)^{2} \operatorname{div} w\right) \mathrm{d} x \mathrm{~d} t+\int u_{0}(x) \cdot w(0, x) \mathrm{d} x=0$,

where we have used Lemma 4.6 and

$$
\begin{aligned}
& \int_{0}^{T^{*}} \int \varepsilon\left(\left(h^{\varepsilon}\right)^{2} L u^{\varepsilon}-\frac{2 \delta}{\delta-1} h^{\varepsilon} \psi^{\varepsilon} \cdot Q\left(u^{\varepsilon}\right)\right) \cdot w \mathrm{~d} x \mathrm{~d} t \\
& \quad \leq C \varepsilon\left(\left\|h^{\varepsilon}\right\|_{L^{\infty}(\operatorname{supp} w)}^{2}\left|\nabla^{2} u^{\varepsilon}\right|_{2}+\left\|h^{\varepsilon}\right\|_{L^{\infty}(\operatorname{supp} w)}\left|\psi^{\varepsilon}\right|_{\infty}\left|\nabla u^{\varepsilon}\right|_{2}\right)|w|_{2} \rightarrow 0 \quad \text { as } \varepsilon \rightarrow 0 .
\end{aligned}
$$

Similarly, we can use the same argument to show that $\left(c^{*}, u^{*}\right)$ also satisfies the equation in (6.3) for $c=\sqrt{A \gamma} \rho^{\frac{\gamma-1}{2}}$ and the initial condition in the sense of distributions. Thus, $\left(c^{*}, u^{*}\right)$ is a weak solution of the Cauchy problem (6.3) in the sense of distributions satisfying the following regularity:

$$
\left(c^{*}, u^{*}\right) \in L^{\infty}\left(\left[0, T_{*}\right] ; H^{3}\right) .
$$


5. Finally, the uniqueness obtained in [43] yields that the whole family $\left(c^{\varepsilon}, u^{\varepsilon}\right)$ converges to $(c, u)=\left(c^{*}, u^{*}\right)$ in the sense of distributions or the strong convergence shown in (6.5).

This completes the proof of Theorem 2.2.

The proof of Corollary 2.1 is similar to that of Theorem 2.2. Here we omit its details.

\subsection{Proof of Corollary 2.2}

We divide the proof into three steps.

1. Some auxiliary functions. First, let $\left(\rho_{0}, u_{0}\right)$ satisfy

$$
\rho_{0} \geq 0, \quad \rho_{0}^{\frac{\gamma-1}{2}} \in H^{3}, \quad u_{0} \in H^{3} .
$$

Next, denote

for some constant $a$ satisfying

$$
f(x)=\frac{1}{1+|x|^{2 a}}
$$

$$
\frac{3}{2(\gamma-1)}<a<\frac{1}{2(1-\delta)} .
$$

It is easy to check that $f(x)$ satisfies

$$
f>0, \quad f^{\frac{\gamma-1}{2}} \in H^{3}, \quad \nabla f^{\frac{\delta-1}{2}} \in D^{1} \cap D^{2}, \quad \nabla f^{\frac{\delta-1}{4}} \in L^{4} .
$$

Finally, let $\chi(x) \in C_{c}^{\infty}\left(\mathbb{R}^{3}\right)$ be a truncation function satisfying (2.6) and denote $\chi_{\varepsilon^{q}}(x)=$ $\chi\left(\varepsilon^{q} x\right)$ for $x \in \mathbb{R}^{3}$. Then we can define the functions $\left(\rho_{0}^{\varepsilon}, u_{0}^{\varepsilon}\right)$ by

$$
\left(\rho_{0}^{\varepsilon}\right)^{\frac{\gamma-1}{2}}=\rho_{0}^{\frac{\gamma-1}{2}} \chi_{\varepsilon^{q}}+\varepsilon^{r} f^{\frac{\gamma-1}{2}}, \quad u_{0}^{\varepsilon}=u_{0}
$$

where $q$ and $r$ are both positive constants to be determined later.

2. The uniform bound of $\left\|\left(\rho_{0}^{\varepsilon}\right)^{\frac{\gamma-1}{2}}\right\|_{3}$ and strong convergence. We use $C>0$ to denote a constant depending only on $\left(\rho_{0}, u_{0}, f\right), \delta$, and $\gamma$ in the rest of this section.

First, we have the formula

$$
\begin{aligned}
& \partial_{x}^{\zeta}\left(\rho_{0}^{\frac{\gamma-1}{2}} \chi_{\varepsilon^{q}}\right)-\rho_{0}^{\frac{\gamma-1}{2}} \partial_{x}^{\zeta} \chi_{\varepsilon^{q}}-\partial_{x}^{\zeta} \rho_{0}^{\frac{\gamma-1}{2}} \cdot \chi_{\varepsilon^{q}} \\
& \quad=\sum_{1 \leq i, j, k \leq 3} l_{i j k}\left(C_{1 i j k} \partial_{x}^{\zeta^{i}} \rho_{0}^{\frac{\gamma-1}{2}} \cdot \partial_{x}^{\zeta^{j}+\zeta^{k}} \chi_{\varepsilon^{q}}+C_{2 i j k} \partial_{x}^{\zeta^{j}+\zeta^{k}} \rho_{0}^{\frac{\gamma-1}{2}} \cdot \partial_{x}^{\zeta^{i}} \chi_{\varepsilon^{q}}\right),
\end{aligned}
$$

where $\zeta=\zeta^{1}+\zeta^{2}+\zeta^{3}$ for three multi-indexes $\zeta^{i} \in \mathbb{R}^{3}, i=1,2,3$, satisfying $\left|\zeta^{i}\right|=0$ or 1 , and $C_{1 i j k}$ and $C_{2 i j k}$ are all constants.

Then it is direct to show

$$
\begin{aligned}
\left|\nabla^{k} \chi_{\varepsilon^{q}}\right|_{\infty} \leq C & & \text { for } k=0,1,2,3, \\
\left|\nabla^{k} \chi_{\varepsilon^{q}} g\right|_{\infty} \rightarrow 0 & \text { as } \varepsilon \rightarrow 0 & \text { for } k=1,2,3, \\
\int_{|x| \geq \frac{1}{\varepsilon^{q}}}\left|\nabla^{k} \rho_{0}^{\frac{\gamma-1}{2}}\right|^{2} \mathrm{~d} x \rightarrow 0 & \text { as } \varepsilon \rightarrow 0 & \text { for } k=0,1,2,3,
\end{aligned}
$$


which, together with (6.12), implies that $\left\|\left(\rho_{0}^{\varepsilon}\right)^{\frac{\gamma-1}{2}}\right\|_{3} \leq C$ and

$$
\left\|\left(\rho_{0}^{\varepsilon}\right)^{\frac{\gamma-1}{2}}-\rho_{0}^{\frac{\gamma-1}{2}}\right\|_{3} \rightarrow 0 \quad \text { as } \varepsilon \rightarrow 0 .
$$

3. The uniform bound of $\varepsilon^{\frac{1}{2}}\left\|\psi_{0}^{\varepsilon}\right\|_{D^{1} \cap D^{2}}+\varepsilon^{\frac{1}{4}}\left|n_{0}^{\varepsilon}\right|_{4}$ with $n_{0}^{\varepsilon}=\nabla\left(\rho_{0}^{\varepsilon}\right)^{\frac{\delta-1}{4}}$. From the definition of $\left(\rho_{0}^{\varepsilon}\right)^{\frac{\gamma-1}{2}}$, we have

$$
\begin{aligned}
& \psi_{0}^{\varepsilon}=\nabla\left(\rho_{0}^{\varepsilon}\right)^{\frac{\delta-1}{2}}=\iota\left(\rho_{0}^{\frac{\gamma-1}{2}} \chi_{\varepsilon^{q}}+\varepsilon^{r} f^{\frac{\gamma-1}{2}}\right)^{\iota-1}\left(\nabla\left(\rho_{0}^{\frac{\gamma-1}{2}} \chi_{\varepsilon^{q}}\right)+\varepsilon^{r} \nabla f^{\frac{\gamma-1}{2}}\right), \\
& n_{0}^{\varepsilon}=\nabla\left(\rho_{0}^{\varepsilon}\right)^{\frac{\delta-1}{4}}=\frac{\iota}{2}\left(\rho_{0}^{\frac{\gamma-1}{2}} \chi_{\varepsilon^{q}}+\varepsilon^{r} f^{\frac{\gamma-1}{2}}\right)^{\frac{\iota}{2}-1}\left(\nabla\left(\rho_{0}^{\frac{\gamma-1}{2}} \chi_{\varepsilon^{q}}\right)+\varepsilon^{r} \nabla f^{\frac{\gamma-1}{2}}\right) .
\end{aligned}
$$

Denote $B_{\varepsilon, q}=B_{\frac{2}{\varepsilon^{q}}}$ and $B_{\varepsilon, q}^{C}=B_{\frac{2}{\varepsilon^{q}}}^{C}=\mathbb{R}^{3} / B_{\frac{2}{\varepsilon^{q}}}$ in the rest of this section for simplicity, and

$$
\begin{aligned}
\left\|\psi_{0}^{\varepsilon}\right\|_{D^{1} \cap D^{2}} & =\left\|\psi_{0}^{\varepsilon}\right\|_{D^{1} \cap D^{2}\left(B_{\varepsilon, q}\right)}+\left\|\psi_{0}^{\varepsilon}\right\|_{D^{1} \cap D^{2}\left(B_{\varepsilon, q}^{C}\right)}, \\
\left|n_{0}^{\varepsilon}\right|_{4} & =\left\|n_{0}^{\varepsilon}\right\|_{L^{4}\left(B_{\varepsilon, q}\right)}+\left\|n_{0}^{\varepsilon}\right\|_{L^{4}\left(B_{\varepsilon, q}^{C}\right)} .
\end{aligned}
$$

Notice that

$$
f(x) \geq \frac{\varepsilon^{2 a q}}{\varepsilon^{2 a q}+2^{2 a}} \quad \text { for } x \in B_{\varepsilon, q}
$$

Then it follows that

$$
\begin{aligned}
\varepsilon^{\frac{1}{4}}\left|n_{0}^{\varepsilon}\right|_{4}= & \varepsilon^{\frac{1}{4}}\left\|n_{0}^{\varepsilon}\right\|_{L^{4}\left(B_{\varepsilon, q}\right)}+\varepsilon^{\frac{1}{4}}\left\|n_{0}^{\varepsilon}\right\|_{L^{4}\left(B_{\varepsilon, q}^{C}\right)} \\
\leq & C \varepsilon^{\frac{1}{4}}\left\|\left(\varepsilon^{r}\left(\frac{\varepsilon^{2 a q}}{\varepsilon^{2 a q}+2^{2 a}}\right)^{\frac{\gamma-1}{2}}\right)^{\frac{\iota}{2}-1}\left(\nabla\left(\rho_{0}^{\frac{\gamma-1}{2}} \chi_{\varepsilon^{q}}\right)+\varepsilon^{r} \nabla f^{\frac{\gamma-1}{2}}\right)\right\|_{L^{4}\left(B_{\varepsilon, q}\right)} \\
& +C \varepsilon^{\frac{1}{4}}\left\|\left(\varepsilon^{r} f^{\frac{\gamma-1}{2}}\right)^{\frac{\iota}{2}-1} \varepsilon^{r} \nabla f^{\frac{\gamma-1}{2}}\right\|_{L^{4}\left(B_{\varepsilon, q}^{C}\right)} \\
\leq & C \varepsilon^{\frac{1}{4}-(r+a q(\gamma-1))\left(1-\frac{\iota}{2}\right)} \leq C
\end{aligned}
$$

under the condition

$$
0<r+a q(\gamma-1)<\frac{1}{4-2 \iota} .
$$

Next, for $\psi_{0}^{\varepsilon}$, we have

$$
\begin{aligned}
\nabla \psi_{0}^{\varepsilon}= & \iota\left(\rho_{0}^{\frac{\gamma-1}{2}} \chi_{\varepsilon^{q}}+\varepsilon^{r} f^{\frac{\gamma-1}{2}}\right)^{\iota-1}\left(\nabla^{2}\left(\rho_{0}^{\frac{\gamma-1}{2}} \chi_{\varepsilon^{q}}\right)+\varepsilon^{r} \nabla^{2} f^{\frac{\gamma-1}{2}}\right) \\
& +\iota(\iota-1)\left(\rho_{0}^{\frac{\gamma-1}{2}} \chi_{\varepsilon^{q}}+\varepsilon^{r} f^{\frac{\gamma-1}{2}}\right)^{\iota-2}\left(\nabla\left(\rho_{0}^{\frac{\gamma-1}{2}} \chi_{\varepsilon^{q}}\right)+\varepsilon^{r} \nabla f^{\frac{\gamma-1}{2}}\right)^{2}, \\
\nabla^{2} \psi_{0}^{\varepsilon}= & \iota\left(\rho_{0}^{\frac{\gamma-1}{2}} \chi_{\varepsilon^{q}}+\varepsilon^{r} f^{\frac{\gamma-1}{2}}\right)^{\iota-1}\left(\nabla^{3}\left(\rho_{0}^{\frac{\gamma-1}{2}} \chi_{\varepsilon^{q}}\right)+\varepsilon^{r} \nabla^{3} f^{\frac{\gamma-1}{2}}\right) \\
& +3 \iota(\iota-1)\left(\rho_{0}^{\frac{\gamma-1}{2}} \chi_{\varepsilon^{q}}+\varepsilon^{r} f^{\frac{\gamma-1}{2}}\right)^{\iota-2} \\
& \times\left(\nabla\left(\rho_{0}^{\frac{\gamma-1}{2}} \chi_{\varepsilon^{q}}\right)+\varepsilon^{r} \nabla f^{\frac{\gamma-1}{2}}\right) \cdot\left(\nabla^{2}\left(\rho_{0}^{\frac{\gamma-1}{2}} \chi_{\varepsilon^{q}}\right)+\varepsilon^{r} \nabla^{2} f^{\frac{\gamma-1}{2}}\right) \\
& +\iota(\iota-1)(\iota-2)\left(\rho_{0}^{\frac{\gamma-1}{2}} \chi_{\varepsilon^{q}}+\varepsilon^{r} f^{\frac{\gamma-1}{2}}\right)^{\iota-3}\left(\nabla\left(\rho_{0}^{\frac{\gamma-1}{2}} \chi_{\varepsilon^{q}}\right)+\varepsilon^{r} \nabla f^{\frac{\gamma-1}{2}}\right)^{3} .
\end{aligned}
$$


Notice that

$$
\begin{aligned}
&\left(f^{\frac{\gamma-1}{2}}\right)^{\iota-1} \nabla^{2} f^{\frac{\gamma-1}{2}}= \frac{1}{\iota}\left(\nabla^{2}\left(f^{\frac{\gamma-1}{2}}\right)^{\iota}-\frac{4(\iota-1)}{\iota}\left(\nabla f^{\frac{\delta-1}{4}}\right)^{2}\right) \\
&\left(f^{\frac{\gamma-1}{2}}\right)^{\iota-1} \nabla^{3} f^{\frac{\gamma-1}{2}=}=\frac{1}{\iota}\left(\nabla^{3}\left(f^{\frac{\gamma-1}{2}}\right)^{\iota}-3 \iota(\iota-1)\left(f^{\frac{\gamma-1}{2}}\right)^{\iota-2} \nabla f^{\frac{\gamma-1}{2}} \nabla^{2} f^{\frac{\gamma-1}{2}}\right. \\
&\left.\quad-\iota(\iota-1)(\iota-2)\left(f^{\frac{\gamma-1}{2}}\right)^{\iota-3}\left(\nabla f^{\frac{\gamma-1}{2}}\right)^{3}\right),
\end{aligned}
$$

which, together with (6.19) and a similar argument used in the derivation of (6.17), implies

$$
\varepsilon^{\frac{1}{2}}\left\|\psi_{0}^{\varepsilon}\right\|_{D^{1} \cap D^{2}} \leq C \varepsilon^{\frac{1}{2}-(r+a q(\gamma-1))(3-\imath)} \leq C,
$$

under the condition

$$
0<r+a q(\gamma-1)<\frac{1}{6-2 \iota} .
$$

Thus, following (6.18) and (6.20), we can just choose

$$
r_{0}=\frac{1}{6(3-\iota)}, \quad q_{0}=\frac{1}{6 a(\gamma-1)(3-\iota)},
$$

and $\left(\rho_{0}^{\varepsilon}, u_{0}^{\varepsilon}\right)$ can be given as

$$
\left(\rho_{0}^{\varepsilon}\right)^{\frac{\gamma-1}{2}}=\rho_{0}^{\frac{\gamma-1}{2}} \chi_{\varepsilon^{q_{0}}}+\varepsilon^{r_{0}}\left(\frac{1}{1+|x|^{2 a}}\right)^{\frac{\gamma-1}{2}}, \quad u_{0}^{\varepsilon}=u_{0},
$$

where $a$ satisfies (6.10).

This completes the proof of Corollary 2.2.

\section{Nonexistence of global solutions with $L^{\infty}$ decay}

This section is devoted to the proof of Theorem 2.3. Denote the total kinetic energy as

$$
E_{\mathrm{k}}(t)=\frac{1}{2} \int\left(\rho|u|^{2}\right)(t, x) \mathrm{d} x .
$$

For simplicity, in this section we denote $\left(\rho^{\varepsilon}, u^{\varepsilon}\right)$ as $(\rho, u)$, and $\left(\rho_{0}^{\varepsilon}, u_{0}^{\varepsilon}\right)$ as $\left(\rho_{0}, u_{0}\right)$.

First, the conservation of momentum can be verified.

Lemma 7.1. Let (2.1) hold and $\varepsilon \geq 0$, and let $(\rho, u)$ be the regular solution obtained in Theorems 1.1 and 2.1. Then

$$
\mathbb{P}(t)=\mathbb{P}(0) \quad \text { for } t \in[0, T] .
$$

Proof. The momentum equations imply

$$
\mathbb{P}_{t}=-\int \operatorname{div}(\rho u \otimes u) \mathrm{d} x-\int \nabla p \mathrm{~d} x+\int \operatorname{div} \mathbb{T} \mathrm{d} x=0,
$$

where we have used the fact that

$$
\left(\rho u \otimes u, \rho^{\gamma}, \rho^{\delta} \nabla u\right) \in W^{1,1}\left(\mathbb{R}^{3}\right) .
$$


Now we are ready to prove Theorem 2.3. Let $T>0$ be any constant. It follows from the definitions of $m(t), \mathbb{P}(t)$, and $E_{\mathrm{k}}(t)$ that

$$
|\mathbb{P}(t)| \leq \int \rho(t, x)|u|(t, x) \mathrm{d} x \leq \sqrt{2 m(t) E_{\mathrm{k}}(t)},
$$

which, together with (5.7) and Lemma 7.1, implies

$$
0<\frac{|\mathbb{P}(0)|^{2}}{2 m(0)} \leq E_{\mathrm{k}}(t) \leq \frac{1}{2} m(0)|u(t)|_{\infty}^{2} \quad \text { for } t \in[0, T] .
$$

Then there exists a positive constant $C_{u}$ such that

$$
|u(t)|_{\infty} \geq C_{u} \quad \text { for } t \in[0, T]
$$

Thus, we have obtained the desired conclusion as shown in Theorem 2.3.

Remark 7.1. In the sense of the three fundamental conservation laws in fluid dynamics, the definition of regular solutions with vacuum is consistent with the physical background of the compressible Navier-Stokes equations. This is the reason why we say in Remark 2.1 that the regular solutions defined above select the fluid velocity in a physically reasonable way.

\section{A. Some basic lemmas}

In this appendix, for self-containedness, we list some basic lemmas that are frequently used in our proof. The first is the well-known Gagliardo-Nirenberg inequality.

Lemma A.1 ([31]). For $p \in[2,6], q \in(1, \infty)$, and $r \in(3, \infty)$, there exists a generic constant $C>0$ that may depend on $q$ and $r$ such that, for any $f \in H^{1}\left(\mathbb{R}^{3}\right)$ and $g \in$ $L^{q}\left(\mathbb{R}^{3}\right) \cap D^{1, r}\left(\mathbb{R}^{3}\right)$,

$$
|f|_{p}^{p} \leq C|f|_{2}^{\frac{6-p}{2}}|\nabla f|_{2}^{\frac{3 p-6}{2}}, \quad|g|_{\infty} \leq C|g|_{q}^{\frac{q(r-3)}{3 r+q(r-3)}}|\nabla g|_{r}^{\frac{3 r}{3 r+q(r-3)}}
$$

Some special cases of the inequalities are

$$
|u|_{6} \leq C|u|_{D^{1}}, \quad|u|_{\infty} \leq C|u|_{6}^{\frac{1}{2}}|\nabla u|_{6}^{\frac{1}{2}}, \quad|u|_{\infty} \leq C\|u\|_{W^{1, r}} .
$$

The second lemma is some compactness results obtained via the Aubin-Lions lemma.

Lemma A.2 ([51]). Let $X_{0} \subset X \subset X_{1}$ be three Banach spaces. Suppose that $X_{0}$ is compactly embedded in $X$, and $X$ is continuously embedded in $X_{1}$. Then the following statements hold:

(i) If $J$ is bounded in $L^{p}\left([0, T] ; X_{0}\right)$ for $1 \leq p<\infty$, and $\frac{\partial J}{\partial t}$ is bounded in $L^{1}\left([0, T] ; X_{1}\right)$, then $J$ is relatively compact in $L^{p}([0, T] ; X)$. 
(ii) If $J$ is bounded in $L^{\infty}\left([0, T] ; X_{0}\right)$ and $\frac{\partial J}{\partial t}$ is bounded in $L^{p}\left([0, T] ; X_{1}\right)$ for $p>1$, then $J$ is relatively compact in $C([0, T] ; X)$.

The following three lemmas contain some Sobolev inequalities on the product estimates, the interpolation estimates, the commutator inequality, etc., which can be found in many references; cf. Majda ([41]). We omit their proofs.

Lemma A.3. Let functions $u, v \in H^{s}$ and $s \geq 2$. Then $u \cdot v \in H^{s}$, and there exists $a$ constant $C_{s}$ depending only on such that

$$
\|u \cdot v\|_{s} \leq C_{s}\|u\|_{s}\|v\|_{s}
$$

Lemma A.4. Let $u \in H^{s}$. Then, for any $s^{\prime} \in[0, s]$, there exists a constant $C_{s}$ depending only on such that

$$
\|u\|_{s^{\prime}} \leq C_{s}\|u\|_{0}^{1-\frac{s^{\prime}}{s}}\|u\|_{s}^{\frac{s^{\prime}}{s}} .
$$

Lemma A.5. Let $r, a$, and $b$ be constants such that

$$
\frac{1}{r}=\frac{1}{a}+\frac{1}{b}, \quad 1 \leq a, b, r \leq \infty
$$

Then, for any $s \geq 1$, if $f, g \in W^{s, a} \cap W^{s, b}\left(\mathbb{R}^{3}\right)$,

$$
\begin{aligned}
& \left|\nabla^{s}(f g)-f \nabla^{s} g\right|_{r} \leq C_{s}\left(|\nabla f|_{a}\left|\nabla^{s-1} g\right|_{b}+\left|\nabla^{s} f\right|_{b}|g|_{a}\right), \\
& \left|\nabla^{s}(f g)-f \nabla^{s} g\right|_{r} \leq C_{s}\left(|\nabla f|_{a}\left|\nabla^{s-1} g\right|_{b}+\left|\nabla^{s} f\right|_{a}|g|_{b}\right),
\end{aligned}
$$

where $C_{s}>0$ is a constant depending only on $s, \nabla^{s} f$ is the set of all $\partial_{x}^{\zeta} f$ with $|\zeta|=s \geq 1$, and $\zeta=\left(\zeta_{1}, \zeta_{2}, \zeta_{3}\right) \in \mathbb{R}^{3}$ is a multi-index.

The final lemma is useful to improve weak convergence to strong convergence.

Lemma A.6. If the function sequence $\left\{w_{n}\right\}_{n=1}^{\infty}$ converges weakly to $w$ in a Hilbert space $X$, then it converges strongly to $w$ in $X$ if and only if

$$
\|w\|_{X} \geq \lim \sup _{n \rightarrow \infty}\left\|w_{n}\right\|_{X}
$$

Funding. The research of Geng Chen was supported in part by the US National Science Foundation Grant DMS-1715012. The research of Gui-Qiang G. Chen was supported in part by the UK Engineering and Physical Sciences Research Council Awards EP/L015811/1 and EP/V008854/1, and the Royal Society-Wolfson Research Merit Award (UK). The research of Shengguo Zhu was supported in part by the China National Natural Science Foundation under Grant 12101395, the Royal Society-Newton International Fellowships NF170015, the Newton International Fellowships Alumni AL/201021 and AL/211005, the Monash University-Robert Bartnik Visiting Fellowships, and the Shanghai Frontier Science Research Center for Modern Analysis. 


\section{References}

[1] S. Bianchini and A. Bressan, Vanishing viscosity solutions of nonlinear hyperbolic systems. Ann. of Math. (2) 161 (2005), no. 1, 223-342 Zbl 1082.35095 MR 2150387

[2] D. Bresch and B. Desjardins, Existence of global weak solutions for a 2D viscous shallow water equations and convergence to the quasi-geostrophic model. Comm. Math. Phys. 238 (2003), no. 1-2, 211-223 Zbl 1037.76012 MR 1989675

[3] D. Bresch and B. Desjardins, Some diffusive capillary models of Korteweg type. C. R. Acad. Sci. 332 (2004), 881-886 Zbl 1386.76070

[4] D. Bresch, B. Desjardins, and C.-K. Lin, On some compressible fluid models: Korteweg, lubrication, and shallow water systems. Comm. Partial Differential Equations 28 (2003), no. 3-4, 843-868 Zbl 1106.76436 MR 1978317

[5] D. Bresch, B. Desjardins, and G. Métivier, Recent mathematical results and open problems about shallow water equations. In Analysis and simulation of fluid dynamics, pp. 15-31, Adv. Math. Fluid Mech., Birkhäuser, Basel, 2007 Zbl 1291.35001 MR 2331331

[6] D. Bresch and P. Noble, Mathematical derivation of viscous shallow-water equations with zero surface tension. Indiana Univ. Math. J. 60 (2011), no. 4, 1137-1169 Zbl 1251.35067 MR 2975338

[7] D. Bresch and P. Noble, Mathematical justification of a shallow water model. Methods Appl. Anal. 14 (2007), no. 2, 87-117 Zbl 1158.35401 MR 2437099

[8] S. Chapman and T. G. Cowling, The mathematical theory of nonuniform gases. 3rd edn., Camb. Math. Libr., Cambridge University Press, Cambridge, 1990 Zbl 0726.76084 MR 1148892

[9] G. Chen, G.-Q. Chen, and S. Zhu, Formation of singularities and existence of global continuous solutions for the compressible Euler equations. 2019, arXiv:1905.07758. To appear in SIAM J. Math. Anal.

[10] G.-Q. Chen and M. Perepelitsa, Vanishing viscosity limit of the Navier-Stokes equations to the Euler equations for compressible fluid flow. Comm. Pure Appl. Math. 63 (2010), no. 11, 1469-1504 Zbl 1205.35188 MR 2683391

[11] G.-Q. G. Chen and M. Perepelitsa, Vanishing viscosity solutions of the compressible Euler equations with spherical symmetry and large initial data. Comm. Math. Phys. 338 (2015), no. 2, 771-800 Zbl 1323.35136 MR 3351058

[12] G.-Q. G. Chen and M. R. I. Schrecker, Vanishing viscosity approach to the compressible Euler equations for transonic nozzle and spherically symmetric flows. Arch. Ration. Mech. Anal. 229 (2018), no. 3, 1239-1279 Zbl 1406.35245 MR 3814602

[13] Y. Cho, H. J. Choe, and H. Kim, Unique solvability of the initial boundary value problems for compressible viscous fluids. J. Math. Pures Appl. (9) 83 (2004), no. 2, 243-275

Zbl 1080.35066 MR 2038120

[14] P. Constantin, T. D. Drivas, H. Q. Nguyen, and F. Pasqualotto, Compressible fluids and active potentials. Ann. Inst. H. Poincaré Anal. Non Linéaire 37 (2020), no. 1, 145-180 Zbl 1430.35185 MR 4049919

[15] C. M. Dafermos, Hyperbolic conservation laws in continuum physics. Grundlehren Math. Wiss. 325, Springer, Berlin, 2000 Zbl 0940.35002 MR 1763936

[16] M. Ding and S. Zhu, Vanishing viscosity limit of the Navier-Stokes equations to the Euler equations for compressible fluid flow with far field vacuum. J. Math. Pures Appl. (9) 107 (2017), no. 3, 288-314 Zbl 1364.35266 MR 3609208

[17] G. P. Galdi, An introduction to the mathematical theory of the Navier-Stokes equations. 2nd edn., Springer Monogr. Math., Springer, New York, 2011 Zbl 1245.35002 MR 2808162 
[18] Y. Geng, Y. Li, and S. Zhu, Vanishing viscosity limit of the Navier-Stokes equations to the Euler equations for compressible fluid flow with vacuum. Arch. Ration. Mech. Anal. 234 (2019), no. 2, 727-775 Zbl 1428.35358 MR 3995050

[19] P. Germain and P. LeFloch, Finite energy method for compressible fluids: the Navier-StokesKorteweg model. Comm. Pure Appl. Math. 69 (2016), no. 1, 3-61 Zbl 1339.35240 MR 3433629

[20] D. Gilbarg, The existence and limit behavior of the one-dimensional shock layer. Amer. J. Math. 73 (1951), 256-274 Zbl 0044.21504 MR 44315

[21] M. Grassin, Global smooth solutions to Euler equations for a perfect gas. Indiana Univ. Math. J. 47 (1998), no. 4, 1397-1432 Zbl 0930.35134 MR 1687130

[22] C. M. I. O. Guès, G. Métivier, M. Williams, and K. Zumbrun, Navier-Stokes regularization of multidimensional Euler shocks. Ann. Sci. École Norm. Sup. (4) 39 (2006), no. 1, 75-175

Zbl 1173.35082 MR 2224659

[23] B. Haspot, Global bmo ${ }^{-1}\left(\mathbb{R}^{N}\right)$ radially symmetric solution for compressible Navier-Stokes equations with initial density in $L^{\infty}\left(\mathbb{R}^{N}\right) .2019$, arXiv:1901.03143v1

[24] D. Hoff and T.-P. Liu, The inviscid limit for the Navier-Stokes equations of compressible, isentropic flow with shock data. Indiana Univ. Math. J. 38 (1989), no. 4, 861-915 Zbl 0674.76047 MR 1029681

[25] F. Huang, R. Pan, T. Wang, Y. Wang, and X. Y. Zhai, Vanishing viscosity for isentropic NavierStokes equations with density-dependent viscosity. arXiv:1009.3978

[26] X. Huang, J. Li, and Z. Xin, Global well-posedness of classical solutions with large oscillations and vacuum to the three-dimensional isentropic compressible Navier-Stokes equations. Comm. Pure Appl. Math. 65 (2012), no. 4, 549-585 Zbl 1234.35181 MR 2877344

[27] H. Hugoniot, Mémoire sur la propagation du mouvement dans les corps et spécialement dans les gaz parfaits. J. Éc. Polytech., 58 (1889), 1-125 Zbl 21.0958 .01

[28] A. Jüngel, Global weak solutions to compressible Navier-Stokes equations for quantum fluids. SIAM J. Math. Anal. 42 (2010), no. 3, 1025-1045 Zbl 1228.35083 MR 2644915

[29] S. Klainerman and A. Majda, Singular limits of quasilinear hyperbolic systems with large parameters and the incompressible limit of compressible fluids. Comm. Pure Appl. Math. 34 (1981), no. 4, 481-524 Zbl 0476.76068 MR 615627

[30] P. E. Kloeden, Global existence of classical solutions in the dissipative shallow water equations. SIAM J. Math. Anal. 16 (1985), no. 2, 301-315 Zbl 0579.76047 MR 777469

[31] O. A. Ladyženskaja, V. A. Solonnikov, and N. N. Ural'ceva, Linear and quasilinear equations of parabolic type. Transl. Math. Monogr. 23, American Mathematical Society, Providence, RI, 1968 Zbl 0174.15403 MR 0241822

[32] H.-L. Li, J. Li, and Z. Xin, Vanishing of vacuum states and blow-up phenomena of the compressible Navier-Stokes equations. Comm. Math. Phys. 281 (2008), no. 2, 401-444 Zbl 1173.35099 MR 2410901

[33] J. Li and Z. Xin, Global existence of weak solutions to the barotropic compressible NavierStokes flows with degenerate viscosities. 2016, arXiv:1504.06826

[34] T. Li and T. Qin, Physics and partial differential equations. Vol. 1. Society for Industrial and Applied Mathematics (SIAM), Philadelphia, PA; Higher Education Press, Beijing, 2012 Zbl 1266.35001 MR 2985747

[35] Y. Li, R. Pan, and S. Zhu, Recent progress on classical solutions for compressible isentropic Navier-Stokes equations with degenerate viscosities and vacuum. Bull. Braz. Math. Soc. (N.S.) 47 (2016), no. 2, 507-519 Zbl 1360.35148 MR 3514417

[36] Y. Li, R. Pan, and S. Zhu, On classical solutions to 2D shallow water equations with degenerate viscosities. J. Math. Fluid Mech. 19 (2017), no. 1, 151-190 Zbl 1369.35065 MR 3607451 
[37] Y. Li, R. Pan, and S. Zhu, On classical solutions for viscous polytropic fluids with degenerate viscosities and vacuum. Arch. Ration. Mech. Anal. 234 (2019), no. 3, 1281-1334 Zbl 1428.35379 MR 4011697

[38] Y. Li and S. Zhu, Formation of singularities in solutions to the compressible radiation hydrodynamics equations with vacuum. J. Differential Equations 256 (2014), no. 12, 3943-3980 Zbl 1290.35008 MR 3190488

[39] T.-P. Liu and W. Wang, The pointwise estimates of diffusion wave for the Navier-Stokes systems in odd multi-dimensions. Comm. Math. Phys. 196 (1998), no. 1, 145-173 Zbl 0912.35122 MR 1643525

[40] T.-P. Liu, Z. Xin, and T. Yang, Vacuum states for compressible flow. Discrete Contin. Dynam. Systems 4 (1998), no. 1, 1-32 Zbl 0970.76084 MR 1485360

[41] A. Majda, Compressible fluid flow and systems of conservation laws in several space variables. Appl. Math. Sci. 53, Springer, New York, 1984 Zbl 0537.76001 MR 748308

[42] T. Makino and B. Perthame, Sur les solutions à symétrie sphérique de l'équation d'EulerPoisson pour l'évolution d'étoiles gazeuses. Japan J. Appl. Math. 7 (1990), no. 1, 165-170 Zbl 0743.35048 MR 1039243

[43] T. Makino, S. Ukai, and S. Kawashima, Sur la solution à support compact de l'équations d'Euler compressible. Japan J. Appl. Math. 3 (1986), no. 2, 249-257 Zbl 0637.76065 MR 899222

[44] A. Matsumura and T. Nishida, The initial value problem for the equations of motion of viscous and heat-conductive gases. J. Math. Kyoto Univ. 20 (1980), no. 1, 67-104 Zbl 0429.76040 MR 564670

[45] A. Mellet and A. Vasseur, On the barotropic compressible Navier-Stokes equations. Comm. Partial Differential Equations 32 (2007), no. 1-3, 431-452 Zbl 1149.35070 MR 2304156

[46] J. Nash, Le problème de Cauchy pour les équations différentielles d'un fluide général. Bull. Soc. Math. France 90 (1962), 487-497 Zbl 0113.19405 MR 149094

[47] W. Rankine, On the thermodynamic theory of waves of finite longitudinal disturbance. Phi. Trans. Royal Soc. London, 1960 (1870), 277-288

[48] L. Rayleigh, Aerial plane waves of finite amplitude. Proc. Royal Soc. London, 84A (1910), 247-284 Zbl 41.0846 .02

[49] D. Serre, Solutions classiques globales des équations d'Euler pour un fluide parfait compressible. Ann. Inst. Fourier (Grenoble) 47 (1997), no. 1, 139-153 Zbl 0864.35069 MR 1437182

[50] J. Serrin, On the uniqueness of compressible fluid motions. Arch. Rational Mech. Anal. 3 (1959), 271-288 (1959) Zbl 0089.19103 MR 106646

[51] J. Simon, Compact sets in the space $L^{p}(0, T ; B)$. Ann. Mat. Pura Appl. (4) 146 (1987), 65-96 Zbl 0629.46031 MR 916688

[52] G. Stokes, On a difficulty in the theory of sound. Philos. Magazine, 33 (1848), 349-356

[53] A. F. Vasseur and C. Yu, Existence of global weak solutions for 3D degenerate compressible Navier-Stokes equations. Invent. Math. 206 (2016), no. 3, 935-974 Zbl 1354.35115 MR 3573976

[54] Z. Xin and S. Zhu, Global well-posedness of regular solutions to the three-dimensional isentropic compressible Navier-Stokes equations with degenerate viscosities and vacuum. 2018, arXiv:1806.02383. To appear in Adv. Math.

[55] Z. Xin and S. Zhu, Well-posedness of the three-dimensional isentropic compressible NavierStokes equations with degenerate viscosities and far field vacuum. J. Math. Pures Appl. (9) 152 (2021), 94-144 Zbl 07379399 MR 4280833 
[56] T. Yang and H. Zhao, A vacuum problem for the one-dimensional compressible Navier-Stokes equations with density-dependent viscosity. J. Differential Equations 184 (2002), no. 1, $163-$ 184 Zbl 1003.76073 MR 1929151

[57] T. Yang and C. Zhu, Compressible Navier-Stokes equations with degenerate viscosity coefficient and vacuum. Comm. Math. Phys. 230 (2002), no. 2, 329-363 Zbl 1045.76038 MR 1936794

[58] S. Zhu, Existence results for viscous polytropic fluids with degenerate viscosity coefficients and vacuum. J. Differential Equations 259 (2015), no. 1, 84-119 Zbl 1320.35265 MR 3335921

[59] S. Zhu, Well-posedness and singularity formation of isentropic compressible Navier-Stokes equations, Ph.D Thesis, Shanghai Jiao Tong University, 2015

Received 13 February 2020; accepted 7 April 2021.

\section{Geng Chen}

School of Mathematics, University of Kansas, Lawrence, KS 66045, USA; gengchen@ku.edu

\section{Gui-Qiang G. Chen}

Mathematical Institute, University of Oxford, Oxford OX2 6GG, UK; chengq@ maths.ox.ac.uk

\section{Shengguo Zhu}

School of Mathematical Sciences, CMA-Shanghai, and MOE-LSC, Shanghai Jiao Tong University, Shanghai 200240, China; zhushengguo@sjtu.edu.cn 